\title{
TREM2 interacts with TDP-43 and mediates microglial neuroprotection against TDP-43-related neurodegeneration
}

Manling Xie

Mayo Clinic

Yong Liu

Mayo Clinic https://orcid.org/0000-0003-1278-7114

Shuyi Zhao

Mayo Clinic

Lingxin Zhang

Mayo Clinic

Dale Bosco

Mayo Clinic

Yuan-Ping Pang

Mayo Clinic https://orcid.org/0000-0003-0838-2560

Udit Sheth

Mayo Clinic

Yuka Martens

Mayo Clinic, Jacksonville https://orcid.org/0000-0002-5194-4269

$\mathrm{Na}$ Zhao

Mayo Clinic https://orcid.org/0000-0001-8648-9422

Chia-Chen Liu

Mayo Clinic Florida https://orcid.org/0000-0001-7203-3926

\section{Yongxian Zhuang}

Mayo Clinic

Liewei Wang

Mayo Clinic

Dennis Dickson

Mayo Clinic https://orcid.org/0000-0001-7189-7917

Mark Mattson

Johns Hopkins Medicine

\section{Guojun Bu}

Mayo Clinic https://orcid.org/0000-0003-3491-1016

Long-Jun Wu ( $\sim$ Wu.LongJun@mayo.edu ) 
Mayo Clinic https://orcid.org/0000-0001-8019-3380

\section{Article}

Keywords: microglial neuroprotection, TDP-43-related neurodegeneration

Posted Date: January 13th, 2021

DOl: https://doi.org/10.21203/rs.3.rs-133413/v1

License: (c) (i) This work is licensed under a Creative Commons Attribution 4.0 International License. Read Full License

Version of Record: A version of this preprint was published at Nature Neuroscience on December 16th, 2021. See the published version at https://doi.org/10.1038/s41593-021-00975-6. 
TREM2 interacts with TDP-43 and mediates microglial neuroprotection against TDP-43-related neurodegeneration

Manling Xie ${ }^{1,2 \#}$, Yong U. Liu ${ }^{1, \# \star}$, Shunyi Zhao ${ }^{1}$, Lingxin Zhang ${ }^{3}$, Dale B. Bosco ${ }^{1}$, Yuan-Ping Pang ${ }^{4}$, Udit Sheth ${ }^{2,5}$, Yuka A. Martens ${ }^{5}$, Na Zhao ${ }^{5}$, Chia-Chen Liu ${ }^{5}$, Yongxian Zhuang $^{3}$, Liewei Wang ${ }^{3}$, Dennis W. Dickson ${ }^{5}$, Mark P. Mattson ${ }^{6}$, Guojun Bu ${ }^{5}$ and Long-Jun $\mathrm{Wu}^{1,5,7 \text { * }}$

${ }^{1}$ Department of Neurology, Mayo Clinic, Rochester, MN, USA.

${ }^{2}$ Mayo Clinic Graduate School of Biomedical Sciences, Rochester, MN, USA.

${ }^{3}$ Division of Clinical Pharmacology, Department of Molecular Pharmacology and Experimental Therapeutics, Mayo Clinic, Rochester, MN, USA.

${ }^{4}$ Computer-Aided Molecular Design Laboratory, Mayo Clinic, Rochester, MN, USA.

${ }^{5}$ Department of Neuroscience, Mayo Clinic, Jacksonville, FL, USA.

${ }^{6}$ Department of Neuroscience, Johns Hopkins University School of Medicine, Baltimore, MD, USA.

${ }^{7}$ Department of Immunology, Mayo Clinic, Rochester, MN, USA.

\# equal contribution

${ }^{*}$ Correspondence:

Long-Jun $\mathrm{Wu}, \mathrm{PhD}$

wu.longjun@mayo.edu

Yong U. Liu, PhD

liuy0321@gmail.com

Department of Neurology, Mayo Clinic

200 First Street SW, Rochester, MN 55905

TEL: (617) 943-7822 


\section{Abstract}

Triggering receptor expressed on myeloid cell 2 (TREM2) is a surface receptor that, in the central nervous system, is exclusively expressed on microglia. TREM2 variants have been linked to increased risk for neurodegenerative diseases, but the functional effects of microglial TREM2 remain largely unknown. To this end, we investigated TAR-DNA binding protein $43 \mathrm{kDa}$ (TDP-43)-related neurodegenerative disease via viral-mediated expression of human TDP-43 protein (hTDP-43) in neonatal and adult mice or inducible expression of hTDP43 with defective nuclear localization signals in transgenic mice. We found that TREM2 deficiency impaired microglia phagocytic clearance of pathological TDP-43, and enhanced neuronal damage and motor function impairments. Mass cytometry analysis revealed that hTDP-43 induced a TREM2-dependent subpopulation of microglia with high CD11c expression and higher phagocytic ability. Using mass spectrometry, we further demonstrated an interaction between TDP-43 and TREM2, in vitro and in vivo, in hTDP-43-expressing transgenic mouse brains. We computationally identified the region within hTDP-43 that interacts with TREM2 and observed the potential interaction in ALS patient tissues. Our data reveal the novel interaction between TREM2 and TDP-43, highlighting that TDP-43 is a potential ligand for microglial TREM2 and the interaction mediates neuroprotection of microglial TREM2 in TDP-43-related neurodegeneration. 


\section{Introduction}

Microglia account for $5-10 \%$ of glial cells and serve as the principal immune cells of the central nervous system (CNS). Initially implicated as inflammatory cells, microglia are now recognized to play neuroprotective roles in neurodegenerative diseases, by sensing their environment, clearing injury stimuli via phagocytosis and preserving neuronal health ${ }^{1}$. Triggering receptor expressed on myeloid cell 2 (TREM2) is exclusively expressed on microglia in the CNS and is critical for microglial proliferation, migration, and phagocytosis ${ }^{2}$. Multiple lines of evidence support the critical role of TREM2 in neurodegenerative diseases. In particular, TREM2 variants are genetically linked to increased risk for Alzheimer's disease $(A D)^{3,4}$. In an AD mouse model, TREM2 was demonstrated to act as a sensor for anionic lipids associated with amyloid- $\beta$ (A $\beta$ ) accumulation or degenerating neurons ${ }^{5}$. In addition, recent studies have further demonstrated, both in vivo and in vitro, that TREM2 is a receptor for $A \beta^{6}$.

TAR-DNA binding protein $43 \mathrm{kDa}$ (TDP-43) was first identified in 1995 as a DNA binding protein and plays a critical role in regulating gene expression ${ }^{7}$. It is the main component of the insoluble and ubiquitinated protein aggregates found in most ALS patients ${ }^{8}$. TDP-43 aggregates have also been identified in other neurodegenerative diseases, such as frontotemporal dementia (FTD) and $A D{ }^{9}$. Therefore, understanding the basis of neurotoxicity related to TDP-43 aggregates might be pertinent for CNS neurodegenerative diseases in general. In epidemiologic studies, whether TREM2 variants are risk factors for ALS is still under debate. A large population based study revealed that TREM2 variant $\mathrm{R} 47 \mathrm{H}$ increases the risk for sporadic ALS ${ }^{10}$. However, other groups found that TREM2 R47H is not associated with ALS ${ }^{11}$. Notably, all these studies focused on TREM2 variant $\mathrm{R} 47 \mathrm{H}$, while other TREM2 variants are largely understudied. Nevertheless, spatial gene expression analysis in both ALS mouse model 
and sporadic ALS patients implicates TREM2-mediated mechanism in disease pathogenesis ${ }^{12,13}$. In addition, the level of soluble TREM2 in cerebrospinal fluid (CSF) of ALS patients was shown to have a strong positive correlation with disease progression in late stage ${ }^{14}$. However, whether or how TREM2 participates in TDP-43-related neurodegeneration remains unknown.

Towards this end, we used viral-transduction to overexpress human TDP-43 (hTDP-43) in the mouse CNS as well as a transgenic mouse model that inducibly expresses hTDP43 bearing defective nuclear localization signal (rNLS8) ${ }^{15}$. We found that TREM2 mediates microglial phagocytic clearance of pathological hTDP-43 proteins and that TREM2 deficiency facilitates motor neuron degeneration. We further demonstrated that microglial TREM2 interacts with pathological hTDP-43, suggesting a potential molecular mechanism underlying the neuroprotective function for microglia in TDP-43-mediated neurodegeneration.

\section{Results}

TREM2 deficiency facilitates hTDP-43-induced behavioral deficits and neurodegeneration.

Intracerebroventricular (ICV) injection of adeno-associated virus (AAV) into postnatal day $0(\mathrm{P} 0)$ mice results in widespread transduction of neurons in the brain. We injected AAV9 into P0 neonatal mice to express hTDP-43 protein fused with a GFP tag (AAV9.CAG.hTDP43-GFP) (Fig. 1a). In this neonatal ICV injection model, the hTDP-43 protein was widely expressed throughout the brain and spinal cord 21 days after AAV injection, including motor cortex, hippocampus, thalamus and brainstem (Extended Data Fig. 1a-d). Immunostaining for NeuN, Iba1, GFAP and CNPase (markers for 
neurons, microglia, astrocytes and oligodendroglia, respectively) indicated that hTDP-43 was exclusively expressed in neurons (Extended Data Fig. 1e, f). Moreover, In line with the clinical characteristics of hTDP-43 pathology, we found hTDP-43 expression in both the nucleus and cytoplasm of neurons 5 weeks after AAV injection (Fig. 1b, c). In addition, pathological inclusions of phosphorylated hTDP-43 (p-hTDP-43) were also detected using p409/410 antibodies at this time point, with more severe pathology in motor cortex (Fig. 1d, Extended Data Fig. 1g). We also observed significant motor deficits, as illustrated by dramatically impaired hindlimb clasping in hTDP-43 expressing mice at day 14 when compared with control mice (injected with AAV9.GFP) (Fig. 1e). These findings indicate that neonatal ICV injection of hTDP-43 virus can induce typical TDP43 pathology and motor deficits in WT mice.

To evaluate the function of TREM2 in hTDP-43-related motor deficits, we compared behavioral outcomes in TREM2 knockout (KO) and wild-type (WT) mice expressing hTDP-43. We found that mortality after hTDP-43 overexpression was significantly higher in TREM2 KO mice than that in WT mice (Fig. 1f). Additionally, TREM2 KO mice expressing hTDP-43 exhibited hindlimb clasping 5 days earlier than WT mice (Fig. 19). Of note, TREM2 KO mice remained hunched in the ensuing observation period, while this behavioral trait began to recover in WT mice approximately 40 days post-virus injection (Fig. 19). These results indicate worse hTDP-43 related motor dysfunction in TREM2 KO mice when compared with WT mice.

Interestingly, at 5 weeks post-virus injection, WT and TREM2 KO mice have similar hindlimb clasping response (Fig. 1g) and weight loss (Extended Data Fig. 2a). However, we found that locomotor impairment was significantly greater in TREM2 KO mice than in WT mice (reduced travel distance in open field testing, Extended Data Fig. 2b, c). 
Interestingly, travel distance by WT mice expressing hTDP-43 was slightly greater than in WT mice expressing control virus (Extended Data Fig. 2b, c). A similar behavioral phenotype was reported for the rTg4510 mouse model of tauopathy ${ }^{16}$. Rotarod performance during the recovery phase (10 weeks post-injection) also revealed greater impairment of motor function (i.e., reduction in latency to fall) in hTDP-43 expressing TREM2 KO mice than in WT mice (Fig. 1h). To study the association of hTDP-43-induced motor impairment with neurodegeneration, we performed Nissl staining at 5 weeks post-virus injection. Shrinkage of neuronal cell populations was detected in both WT and TREM2 KO mice expressing hTDP-43, with lower neuronal numbers in TREM2 KO mice (Fig. 1i, j). Similarly, NeuN immunostaining showed that NeuN loss in the primary motor cortex was greater in TREM2 KO mice than in WT mice expressing hTDP-43 (Extended Data Fig. 2d, e).

To determine whether motor function recovery at later time points correlated with levels of pathological hTDP-43 protein, we isolated soluble and sarkosyl-insoluble fractions from whole brains to measure the total hTDP-43 and phosphorylated hTDP-43 (p-hTDP-43) protein levels at 10 weeks post-virus injection (Extended Data Fig. 3a). Pathological TDP-43 aggregates resist solubilization in sarkosyl ${ }^{17}$. p-TDP-43 has been reported to be one of the major components of the aggregated inclusions critical for ALS progression 18,19. Western blot analysis revealed that levels of both hTDP-43 and p-hTDP-43 were much higher in TREM2 KO mice when compared to WT mice in the soluble fraction (Extended Data Fig. 3b). Phospho-Ser409/410 antibody also detected a $\sim 20 \mathrm{kD}$ fragment in the soluble fractions (Extended Data Fig. 3b). Notably, Ser409/410 is at the extreme C-terminal of TDP-43 protein, suggesting that either the full length hTDP-43 was cleaved to generate C-terminal fragments in TREM2 KO mice, or the C-terminal fragments were less efficiently cleared by TREM2 KO mice. The 
sarkosyl-insoluble fraction in brains of TREM2 KO mice also contained more p-hTDP-43 (Extended Data Fig. 3c). Together, these results indicate that the higher level of pathological hTDP-43 in the brains of TREM2 KO mice likely contributes to their more severe, relatively irreversible motor dysfunction compared with WT mice.

\section{TREM2 deficiency abolishes the hTDP-43-induced CD11c $^{+}$microglia subpopulation.}

As the CNS resident immune cells, microglia undergo significant phenotypic changes following CNS injury and neurodegeneration ${ }^{20}$. We therefore evaluated the effects of TREM2 deficiency on microglial phenotypes in response to hTDP-43-related neurodegeneration. We employed an integrated set of cell surface antibodies to enable mapping of microglial phenotypes via mass cytometry (CyTOF), a high dimensional single cell analysis method that can identify functional cell subpopulations ${ }^{21}$. Microglia antibody panel based on surface markers were listed in supplementary Table1. The microglial subpopulations and phenotypic changes were further analyzed by immunohistochemistry (Fig. 2a).

For CyTOF analysis, we first gated $\mathrm{CD} 45^{+} \mathrm{CD} 11 \mathrm{~b}^{+}$myeloid cells from the brain of WT mice and then used a t-SNE map to obtain an overview of brain myeloid cells (Fig. 2b). The $\mathrm{CD} 45^{+} \mathrm{CD} 11 \mathrm{~b}^{+}$sub-population of microglia was further categorized by FlowSOM-guided clustering with expression of CX3CR1 (Fig. 2c). Both t-SNE map and FlowSOM-guided clustering analysis revealed a major microglia population characterized by $\mathrm{CD} 45^{\mathrm{mid}}: \mathrm{CD} 11 \mathrm{~b}^{+}: \mathrm{CX} 3 \mathrm{CR} 1^{\mathrm{hi}}: \mathrm{F} 4 / 80^{+}: \mathrm{CD}^{+} 4^{+}: \mathrm{MeTK}^{+}:$Siglec- $\mathrm{H}^{+}: \mathrm{CD} 11 \mathrm{c}^{-}$ signature (Fig. 2d).

Frequency analysis of microglia based on manual gating revealed that numbers of 
microglia in the primary motor cortex were similarly increased in both WT and TREM2 KO mice expressing hTDP-43 at 5 weeks post-virus injection (Fig. 2e). These results are consistent with hTDP-43-increased microglia number using immunostaining analysis in CX3CR $1^{\text {GFP/+}}$ :WT and CX3CR $1^{\text {GFP/+ }}$ :TREM2 KO mice (Extended Data Fig. 4a, b). Transgenic $\mathrm{CX} 3 \mathrm{CR} 1^{\mathrm{GFP} /+}$ mice expressing GFP under the control of the fractalkine promoter allow better visualization of microglial morphology ${ }^{22}$. Quantitative analyses revealed that microglia differed morphologically, with TREM2 KO mice exhibiting less evidence of activation in response to hTDP-43 (i.e., they had smaller soma size; Extended Data Fig. 4c).

Interestingly, spanning-tree progression analysis (SPADE) revealed that sub-clusters of microglia had a distinctive CD11 $c^{\text {high }}$ phenotype, which was only found in WT hTDP-43 expressing mice but not in WT control mice (Fig. 2f). Moreover, in TREM2 KO hTDP-43 expressing mice, CD11 $\mathrm{c}^{\text {high }}$ microglial subpopulation was absent (Fig. 2f). CD11 ${ }^{+}$ microglia have been reported in a mouse model of $A D$ as an activated subpopulation that clusters around amyloid plaques ${ }^{23}$. We next evaluated the brains of hTDP-43 expressing mice for the distribution of $\mathrm{CD}_{11 \mathrm{C}^{+}}$microglia by immunostaining (Fig. $\mathbf{2 g}$ ). Indeed, we found a highly activated microglia subpopulation expressing CD11c in the primary motor cortex of WT mice after hTDP-43 overexpression, while there were few CD11 $^{+}$microglia in TREM2 KO mice expressing hTDP-43 (Fig. 2g, h). To further explore the function of $\mathrm{CD} 11 \mathrm{c}^{+}$microglia, we performed co-staining for Iba1 and CD11c with GFP-hTDP-43. We observed that microglia with phagocytosed GFP-hTDP-43 (phagocytic microglia) had higher CD11c expression and accounted for $7 \%$ of total microglia (Extended Data Fig. 4d, e). Together, our results suggest that TREM2 deficiency abolishes the hTDP-43-induced $\mathrm{CD}_{11 \mathrm{c}^{+}}$subpopulation of microglia, which plays a critical role in phagocytosing hTDP-43. 
TREM2 deficiency locks microglia into a homeostatic status in TDP-43 neurodegeneration.

To investigate how TREM2 deficiency might affect microglial response, we stereotactically injected GFP-hTDP-43 virus into the primary motor cortex of 2-month-old mice and performed a comprehensive analysis of microglial morphology and phagocytosis (Fig. 3a and Extended Data Fig. 5a). At day 14 in this adult local injection model, hTDP-43 inclusions were found predominantly in neuronal nuclei and diffusely in cytoplasm (Extended Data Fig. 5b). hTDP-43 expression in WT and TREM2 KO groups did not differ significantly at day 14 , as revealed by the density analysis of GFP-hTDP-43 particles (Extended Data Fig. 5c, d), indicating similar viral transduction efficiency. This is further confirmed by immunoblotting against GFP or TDP-43 (Extended Data Fig. 5e). As expected, microglia accumulation was abundant in areas of hTDP-43 expression at 14 days post-virus injection of both WT and TREM2 KO mice (Extended Data Fig. 5f, g). In addition, WT microglia exhibited a canonical reactive phenotype, less ramified with enlarged soma and shorter processes in response to hTDP-43 expression (Fig. 3b-e). However, this hTDP-43-induced reactive phenotype was significantly attenuated in TREM2 KO mice (Fig. 3b-e). These data indicate that TREM2 deficiency reduces microglial activation following TDP-43 neurodegeneration.

We next examined two typical microglia homeostatic markers, P2Y12 and TMEM119 ${ }^{24}$, in response to hTDP-43 expression. P2Y12 and TMEM119 were largely confined to microglial processes under basal conditions in both WT and TREM2 KO mice (Fig. 3f, g). We found that hTDP-43 induced remarkable downregulation of P2Y12 and TMEM119 in WT microglia (Fig. 3f-i). However, both P2Y12 and TMEM119 expression were unchanged from baseline in TREM2 KO microglia in response to hTDP-43 (Fig. 3f-i). 
These results suggest that TREM2 deficiency attenuates microglial activation and locks microglia into a homeostatic status in TDP-43 neurodegeneration.

We further characterized CD11 $\mathrm{c}^{+}$microglia subpopulation in hTDP-43 expressing adult mice. Consistent with the neonatal ICV injection model, we found a dramatic upregulation of CD11c in microglia from WT mice in response to local hTDP-43

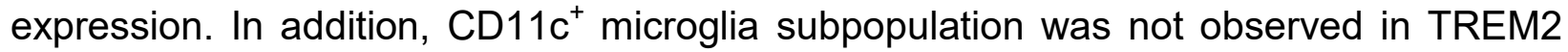
KO mice expressing GFP-hTDP-43 (Fig. 3j). More importantly, we found that CD11c ${ }^{+}$ microglia are mostly associated with GFP-hTDP-43 in WT mice but not in TREM2 KO mice by co-localization analysis (Fig. $\mathbf{3 k}, \mathbf{I}$ ). These results demonstrate that TREM2-dependent CD11c ${ }^{+}$microglia phagocytose pathological hTDP-43 proteins, which manifests the critical function of TREM2 in microglia reactive phenotype towards TDP-43 neurodegeneration.

\section{TREM2 deficiency reduces clearance of pathological hTDP-43 by microglia.}

A recent study suggested that microglia actively participate in clearing hTDP-43 in a transgenic TDP-43 mouse model ${ }^{25}$. Additionally, TREM2 is important for phagocytosis of cell debris from apoptotic neurons and amyloid plaques by microglia ${ }^{26,27}$. We therefore tested whether TREM2 deficiency affects microglia-mediated hTDP-43 clearance. To this end, we first performed immunostaining for CD68, a lysosomal marker upregulated in phagocytic cells, at 2 weeks following hTDP-43 expression in the primary motor cortex. Our results showed that hTDP-43 induced a dramatic increase of CD68 expression in WT but not in TREM2 KO mice (Fig. 4a). The analysis of CD68 and CX3CR1-GFP co-localization further confirmed the upregulation of CD68 in the individual microglia was reduced in TREM2 KO mice (Fig. 4b). In addition, these $\mathrm{CD}^{+} 8^{+}$microglia have high CD11c expression and are co-localized with GFP-hTDP-43 (Fig. 4c). Next, we directly 
investigated whether hTDP-43 was phagocytosed by microglia. We tagged hTDP-43 with GFP and stained microglial with lba1. At 4 weeks following hTDP-43 expression, triple-immunostaining of DAPI, Iba1 and GFP-hTDP-43 showed that hTDP-34 were in the cytoplasm but not nucleus of $\mathrm{Iba}^{+}$microglia (Fig. 4d). A large number of GFP-hTDP-43 were present in $\mathrm{Iba1^{+ }}$ microglia in WT mice, but were rarely observed inside $\mathrm{Iba}^{+}$microglia in TREM2 KO mice (Fig. 4d, e, Extended Data Fig. 5h). Co-localization analysis further confirmed that GFP-hTDP-43 existed in soma of WT microglia, but not TREM2 KO microglia (Fig. 4f). Consistent with the neonatal ICV injection model, we found that phagocytic microglia (colocalized with hTDP-43) had a higher CD11c expression compared with non-phagocytic microglia (Fig. $\mathbf{4 g}, \mathbf{h}$ ). These data further demonstrate that TREM2 is required for hTDP-43-induced CD11c+ subpopulation of microglia which has strong phagocytic functions.

TREM2 deficiency leads to greater brain tissue accumulation of hTDP-43 protein. To characterize the dynamic changes of hTDP-43 pathology resulting from microglial phagocytic clearance, we examined total and p-TDP-43 level at 4 weeks following hTDP-43 expression. Our immunostaining results showed that p-TDP-43 was completely co-localized with GFP-hTDP-43 clusters in both WT and TREM2 KO mice (Fig. 5a, b). However, TREM2 KO mice had significantly more hTDP-43 and p-TDP-43 accumulation than WT mice (Fig. 5c, d). In addition, compared with WT mice, average size of GFP-hTDP-43 clusters was much larger in TREM2 KO mice (Fig. 5e). Particle size distribution analysis revealed that hTDP-43 clusters with size below $40 \mu \mathrm{m}^{2}$ or over 200 $\mu \mathrm{m}^{2}$ were much higher in TREM2 KO than in WT mice (Fig. 5f).

Using Western blot against GFP or TDP-43, we consistently found the higher level of hTDP-43 in TREM2 KO mice than in WT mice in radioimmunoprecipitation assay buffer 
(RIPA)-soluble fractions (Fig. 5g). Immunoblotting against p-hTDP-43 revealed a $\sim 27$ kD fragment in the soluble fraction, the level of which was significantly higher in TREM2 KO mice following hTDP-43 expression. Interestingly, the phosphorylated full length hTDP-43 was not detected in the adult local injection model, suggesting that the cleaved products are more prone to posttranslational modification (Fig. 5g). To test whether the large GFP-hTDP-43 particles with high p-TDP-43 content were aggregated, we then isolated sarkosyl-insoluble fractions from brain tissues and performed Western blot analysis. Indeed, p-hTDP-43 was significantly more abundant in the sarkosyl-insoluble fraction of TREM2 KO brains than in WT brains following hTDP-43 expression (Fig. $\mathbf{5 g}$ ). Consistent with the observations from the soluble fractions, cleaved p-hTDP-43 fragments were the major component of the hyperphosphorylated hTDP-43 (Fig. $\mathbf{5 g}$ ). Together, these results indicate that microglia TREM2 is critical for microglial phagocytosis and clearance of TDP-43. In TREM2 KO mice, the uncleared hTDP-43 clusters are prone to be cleaved and then form hyperphosphorylated aggregates.

We next assessed neuronal loss in the motor cortex via NeuN staining 4 weeks after hTDP-43 expression. Consistent with the neonatal ICV injection model, we found that hTDP-43 expression lead to reduced NeuN staining, which was further aggravated in TREM2 KO mice (Extended Data Fig. 6a, b). By co-staining with Iba1, we found that a large number of microglia in WT mice were located close to presumably damaged neurons, following hTDP-43 expression (Extended Data Fig. 6c, d). However, the proximity of microglia to neurons was unremarkable in TREM2 KO mice. In addition, we found that those neuron-associated microglia had high CD11c expression (Extended Data Fig. 6e), suggesting a phagocytic function. Thus, these results collectively demonstrate that TREM2 deficiency leads to greater brain tissue accumulation of hTDP-43 protein and aggravates TDP-43-induced neurodegeneration. 


\section{TDP-43 interacts with TREM2 in vitro and in vivo.}

The TDP-43 protein in ALS patient blood and CSF is likely released from cells via extracellular vesicles ${ }^{28,29}$. We hypothesize that hTDP-43 released into the CNS parenchyma from degenerating neurons can be detected by microglia through TREM2. To test this hypothesis, we used human iPSC-derived neurons infected with GFP-hTDP-43 virus and evaluated hTDP-43 release. By day 21 after virus infection, hTDP-43 was robustly expressed throughout the cytoplasm and neuronal damage was extensive (Extended Data Fig. 7a). We collected the culture medium and performed Western blot to examine whether hTDP-43 was released extracellularly. Indeed, extracellular hTDP-43 was detectable in the culture medium directly or by capture via bead-immobilized GFP antibody (Extended Data Fig. 7b). Interestingly, by using antibody recognizing N-terminal hTDP-43, we observed cleaved products at around $\sim 25$ $\mathrm{kD}$ in the culture medium input, suggesting a mix of full-length and $\mathrm{N}$-terminal fragments of hTDP-43 were released into the extracellular space (Extended Data Fig. 7b).

Next, to test whether TREM2 can interact with hTDP-43, we performed the co-immunoprecipitation (co-IP) experiments to examine their potential interaction. To this end, we transfected human embryo kidney (HEK) cells with myc-tagged hTREM2 (myc-hTREM2) or control plasmids. hTREM2 was immunoprecipitated using an hTREM2 antibody. Immunoblot against hTDP-43 revealed the co-IP of endogenous hTDP-43 from HEK cells (Fig. 6a). We then performed liquid chromatography with tandem mass spectrometry (LC-MS/MS) to characterize the interaction between TREM2 and TDP-43. Specifically, annotated MS/MS spectra of $V^{124}$ LVEVLADPLDHR $^{136}$ from hTREM2 and $T^{103}$ SDLIVLGLPWK ${ }^{114}$ from hTDP-43 were obtained from anti-hTREM2 immunoprecipitates (Fig. 6b). The two peptides are a fragment of the extracellular 
ligand-binding domain (ECD) of hTREM2 and a fragment of the RNA recognition motif 1 (RRM1) of hTDP-43, respectively. To further explore which domain(s) of hTDP-43 interacted with TREM2, we co-transfected HEK cells with myc-hTREM2 and GFP-hTDP-43 C terminal fragment (residues 216-414) or GFP control plasmids (Extended Data Fig. 7c). GFP or GFP-tagged proteins were captured using bead-immobilized GFP antibody. Immunoblot using anti-Myc revealed the co-IP of GFP-hTDP-43 C terminal fragment with myc-hTREM2, indicating the interaction of hTREM2 with the hTDP-43 C-terminal fragment (Extended Data Fig. 7d). In addition, co-IP using bead-immobilized myc antibody obtained similar results (Extended Data Fig. 7e). Therefore, these results together suggest that hTDP-43 is able to interact with TREM2 in vitro.

To evaluate the possible interaction between TREM2 and TDP-43 in vivo, we used a new inducible mouse model of ALS-rNLS8, which expresses mutant hTDP43 (hTDP43- $\Delta$ NLS) in neurons in a doxycycline (DOX)-dependent manner ${ }^{15}$. We confirmed that mutant hTDP-43 accumulated in cytoplasm after expression (Fig. 6c. d), as the hTDP43- $\Delta$ NLS lacks nuclear localization sequence (NLS). Consistent with previous studies ${ }^{15}$, we observed typical clasping phenotype when hTDP43- $\triangle$ NLS was induced during removal of DOX diet, with a rapid functional recovery as early as 2 weeks back on DOX (Fig. 6e, f). In addition, we found dramatic microglia activation during the recovery period (Fig. $\mathbf{6 g}$, h). We then performed immunoprecipitation using a biotinylated mTREM2 antibody in rNLS8 mouse brain lysates. Immunoblot against mTREM2 and hTDP-43 revealed the co-IP of endogenous mTREM2 with hTDP-43 (Fig. 6i). We then analyzed the anti-mTREM2 immunoprecipitates from rNLS mouse cortex tissue by LC-MS/MS. Similarly, we detected MS/MS spectrum of $N^{99}$ LQAGDAGLYQcQSLR ${ }^{114}$ (c is carbaminomethylated cysteine) from mTREM2. In addition, we obtained peptide 
$\mathrm{F}^{152}$ TEYETQVK ${ }^{160}$ from hTDP-43 in immunoprecipitates, suggesting mTREM2 is physically associated with hTDP-43 (Fig. 6j).

The next plausible question was whether there are potential interactions between TREM2 and TDP-43 in human ALS patients. To this end, we obtained frozen autopsied specimens of ALS patients from Mayo Clinic Brain Bank for Neurodegenerative Disorders and examined the interaction. Basic characteristics of human tissue donors are listed in supplementary Table 2. First, in addition to well-known increased expression of TDP-43 in ALS patients ${ }^{8}$, we found that TREM2 expression in both cortex and spinal cord from ALS patients was greater than those from non-neurological controls (Fig. 7a, b). More importantly, an endogenous hTDP-43/TREM2 complex was co-immunoprecipitated from ALS patient spinal cord tissue lysates (Fig. 7c). Taken together, our results indicate the interactions between TREM2 and hTDP-43 in vivo from both mouse and human tissues.

\section{TREM2 in contact with residues $312-343$ of $h$ TDP-43 in silico}

To further investigate the interaction between TREM2 and hTDP-43 at the atomic level, we performed computational analysis. Because hTDP-43 is intrinsically disordered ${ }^{30}$ and has a high propensity for aggregation ${ }^{31}$, we did not conduct surface plasmon resonance or biolayer interferometry analysis of TREM2 interaction with an intact hTDP-43. Our in vitro study already showed that TREM2 interacted with residues 216-414 of hTDP-43. Therefore, we carried out microsecond molecular dynamics (MD) simulations using forcefield FF12MC and the crystal structures of the hTREM2 ECD and low-complexity domain (LCD) fragments of hTDP-43 ${ }^{32,33}$. The four LCD fragments were $\mathrm{N}^{312} \mathrm{FGAFS}^{317}$ (NFG), $\mathrm{A}^{321} \mathrm{MMAAA}^{326}$ (AMM), $\mathrm{S}^{333} \mathrm{WGMMGMLASQ}^{343}$ (SWG), and $\mathrm{G}^{396}$ FNGGFG $^{402}$ (GFN) (Fig. 7d-e). They were chosen because of their involvement in 
irreversible stress granules ${ }^{33}$. Our visual inspection suggested the structural fitness between the residues in complementarity-determining regions (CDRs) of the TREM2 ECD and those in four LCD fragments of hTDP-43.

Four sets of 20 distinct, independent, unrestricted, unbiased and isobaric-isothermal MD simulations were carried out in silico. Each of the four hTDP-43 LCD fragments was manually docked into the same ECD region to have the LCD fragment surrounded by CDR2 on one side and CDR1 and CDR3 on the other side. We then used the TREM2 ECD in complex with each of the four hTDP-43 LCD fragments. Interestingly, the simulations showed that AMM and SWG moved out of the CDR1-3 to interact with $\beta$-strands residues $75-78$ and $65-70$ of the ECD, respectively (Fig. $\mathbf{7 d}$, e). More interestingly, GFN and NFG remained in the CDR1-3, and NFG (but not GFN) moved closer to R47 of the ECD with its two phenyl rings interacting with W44 and W70 of the ECD (Fig. 7f, g). There was an overlap for the binding sites on the ECD for NFG and GFN (Fig. 7h), but the binding sites for NFG, AMM, and SWG were distinct (Fig. 7h). These results suggest that TREM2 is in contact with the LCD residues $312-343$ that

encompass NFG, AMM, and SWG and constitute a potential epitope of hTDP-43 for TREM2, whereas GFN may represent another epitope. In addition, our simulations revealed that none of the four LCD fragments interact with the two glycosylation sites (N20 and N79) of the ECD domain of TREM2. Therefore, the interaction between TREM2 and hTDP-43 is likely independent of the glycosylation state of the ECD domain.

\section{DISCUSSION}

A major challenge to ALS research is identifying the intrinsic mechanisms that can sense an aberrant CNS environment during the initial stage of disease, and protect motor 
neurons from degeneration ${ }^{34}$. Our study provides a comprehensive analysis of the impact of TREM2 deficiency on microglial phenotypic and functional change in mouse models of TDP-43 proteinopathy. These results demonstrate that TREM2 regulates the microglial response to, and phagocytic clearance of, pathological TDP-43, thus reducing TDP-43 proteinopathy and neurodegeneration. We further reveal that TDP-43 interacts with TREM2 both in vitro and in vivo. This explains why mice with TREM2 deficiency exhibit reduced microglial clearance of TDP-43 and worse motor deficits. Our study suggests a protective role of microglial TREM2 in TDP-43 clearance to alleviate TDP-43 neurodegeneration.

\section{TREM2 mediates microglial activation in the course of TDP-43 pathology}

The intrinsic heterogeneity of microglia allows them to acquire multiple phenotypes in response to extrinsic stimuli ${ }^{35,36}$. Our study provides strong evidence of phenotypic impact of TREM2 on microglia response to TDP-43 pathology. Two recent studies have demonstrated that TREM2 deficiency attenuated microglial responses to $A \beta$ and tau pathologies ${ }^{37,38}$. In contrast, elevated TREM2 gene dosage conferred a rescuing effect in $A D$ mice by altering microglial morphology and reprogramming microglia responsivity 39. Here we found that TREM2 deficiency significantly blocks the microglial activation in response to TDP-43 accumulation. At least two potential mechanisms could underlie TREM2-dependent microglia activation: (1) The direct interaction with pathological TDP-43 activates TREM2 signaling pathway via DNAX-activation protein 12 (DAP12) and phosphorylation of Syk ${ }^{40}$. (2) Neuronal contact-dependent activation is another possibility. This is supported by increased number of microglia-neuron physical interaction in response to TDP-43 pathology. In addition, prior study showed that lack of TREM2 impairs microglial migration towards damaged neurons ${ }^{41}$. Thus, one can presume that TDP-43 damaged neurons express signals sensed by TREM2 that might 
trigger microglial activation. Together, our study shed new light on the "find me" mechanisms that underline TREM2-mediated microglial activation in response to TDP-43 related neurodegeneration.

The next important question to address is what molecular changes occur during microglia phenotypic reprogramming after sensing stimuli. We showed TREM2 deficiency prevents the downregulation of two microglia homeostatic markers P2Y12 and TMEM119 in the context of TDP-43 related neurodegeneration. In addition, microglial morphology was locked into homeostatic states in TREM2 KO mice when compared with WT mice in response to TDP-43. However, how these molecular changes can remodel the cytoskeleton and morphology, underlying phenotypic change, remains unknown. In the context of $A D$ mice, brain transcriptomic analysis demonstrated that elevated TREM2 gene dosage upregulated reactive microglia genes and dampened multiple disease-associated microglia genes ${ }^{39}$. Therefore, it is likely that the activation of microglial TREM2 by TDP-43 may regulate the transcription factors that affect homeostatic gene expression in microglia. Future comprehensive transcriptome-wide and network analysis studies are needed to investigate the potential molecular candidates that could be tapped to regulate microglia activation in the course of TDP-43 pathology.

Interestingly, we found that TREM2 deficiency did not diminish the expansion of microglial numbers in our disease model, which is reminiscent of other microglia receptors involved in proliferation. Previous study demonstrated that TREM2 is important for microglial proliferation in response to KA treatment and promotes microglia survival via $\mathrm{Wnt} / \beta-C a t e n i n$ pathway ${ }^{42}$. Therefore, it is likely that TREM2 may promote microglial proliferation in a context-dependent manner. These results also raised an important 
question as to the major driver for microglia proliferation in response to TDP-43. Previous studies have reported that colony-stimulating factor-1 receptor (CSF1R) is critical in microglial proliferation in response to peripheral nerve injury and in seizures 43,44. In addition, TREM2 and CSF1R share the similar adaptor protein DAP12 and downstream signals ${ }^{45}$. Thus, it would be interesting to explore whether CSF1R mediated pathway can independently serve as a main regulator for microglial proliferation in TDP-43-related neurodegeneration.

\section{TREM2 mediates microglial phagocytic clearance of pathological hTDP-43}

Our study demonstrates that TREM2 can directly mediate microglia phagocytosis of pathological TDP-43 proteins. The presence of hTDP-43 in microglia is unlikely due to the expression of AAV-hTDP-43. AAV virus is known to have very limited ability to infect microglia ${ }^{46}$. Instead, we provided strong evidence that microglia actively phagocytosed pathological hTDP-43 in WT mice which was significantly attenuated in TREM2 KO mice. This is consistent with observations in AD mouse models in which TREM2 mediated microglial phagocytosis can limit amyloid plaque growth ${ }^{37}$. Phagocytosis describes the cellular process of eating, including receptor mediated recognition, engulfment, and degradation of particulate organisms or structures with size over $0.5 \mu \mathrm{m}{ }^{47}$. As professional phagocytes, microglia orchestrate innate brain immune response by eliminating detrimental structures ${ }^{47,48}$. Typical microglia phagocytosis receptors include Toll-like receptors (TLRs) and TREM2. The former has high affinity to bind pathogen-associated molecular patterns (PAMPS), and the latter can recognize apoptotic cellular substances ${ }^{49}$. In addition, other receptors like complement receptors (CR3), pyrimidinergic receptors (P2Y6) and TAM receptors are also involved in microglia phagocytosis ${ }^{50-52}$. Although our results strongly suggest that microglial TREM2 is a key receptor in phagocytosis of TDP-43, it would be of interest to explore whether other 
microglia phagocytosis receptors could work together with TREM2.

Our study sheds novel light on the antigen presenting function of microglia related to the phagocytosis of TDP-43. Microglia have been reported to serve as antigen-presenting cells in response to inflammatory challenge ${ }^{53}$ and to other neurodegenerative diseases, like $A D$ and $P D{ }^{54,55}$. After uptake of the pathological TDP-43 proteins, the further degradation of TDP-43 likely leads to the antigen presentation. We found that microglia do not express CD11c under normal conditions, which is in agreement with the poor antigen presenting ability of microglia in general ${ }^{56}$. However, we identified a distinct microglia subpopulation that highly expressed CD11c in response to hTDP-43 expression in WT mice. This CD11 ${ }^{+}$subpopulation of microglia is dependent on TREM2 and is highly phagocytic towards hTDP-43. In addition, considering the function of CD11c in antigen-presenting cells, these observations raised the question of whether CD11c $\mathrm{c}^{+}$microglia could serve as those cells during TDP-43 related neurodegeneration. Indeed, increased CD4 ${ }^{+} \mathrm{T}$ cell accumulation has been reported in patient with $\mathrm{ALS}^{57}$ and other chronic neurodegeneration pathologies ${ }^{58}$. Moreover, $\mathrm{T}$ cell related immunotherapy has been applied in clinical trial and brings new hope for ALS patients ${ }^{59}$. These lines of evidence warrant future studies on the interaction of the $\mathrm{CD} 11 \mathrm{c}^{+}$microglia and infiltrated T cell in TDP-43 related neurodegeneration.

Importantly, our data showed that TDP-43 induced CD11 $\mathrm{c}^{+}$upregulation is dependent on TREM2, as CD11 $\mathrm{c}^{+}$microglia are absent in TREM2 KO mice. We further demonstrated the beneficial function of $\mathrm{CD} 11 \mathrm{c}^{+}$microglia in clearance of pathological TDP-43 proteins. These findings are consistent with an $A D$ mouse model where strong upregulation of microglial CD11c was significantly attenuated by TREM2 deficiency ${ }^{5}$. The CD11c ${ }^{+}$ microglia are localized near amyloid plaques, potentially to counteract amyloid 
deposition ${ }^{23}$. In other disease models, like a cuprizone-induced demyelination model, $\mathrm{CD}_{11 \mathrm{c}^{+}}$microglia can accelerate the repair of damaged white matter ${ }^{60}$. Our results of TREM2-dependent CD11C expression in response to TDP-43 raised these intriguing questions: (1) how TREM2 regulates microglial CD11c expression, (2) the function of

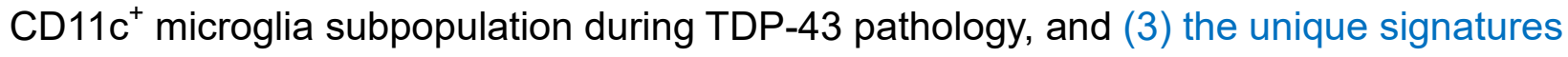
of the $\mathrm{CD}_{11 \mathrm{c}^{+}}$microglia. Previous transcriptional analysis of $\mathrm{CD}_{11 \mathrm{c}^{+}}$microglia accumulating around amyloid plaques revealed a higher expression of TREM2 in a mouse model of Alzheimer's disease ${ }^{23}$. Whether TREM2 is upregulated in CD11c ${ }^{+}$ microglia subpopulation in the context of TDP43 pathology needs further investigation.

We are cognizant of the fact that hTDP-43 overexpression model cannot perfectly mimic ALS patient pathology. Nevertheless, our study still provides insight into the molecular targets of reprogramming microglia via TREM2 in sensing and regulating TDP-43-related neurodegeneration.

\section{TDP-43 interacts with TREM2}

It has been reported that potential ligands for TREM2 are lipids, APOE, and $A \beta^{5,6,61}$. Our present results demonstrate that TDP-43 also interacts with TREM2 both in vitro and in vivo and thus may act as a ligand for TREM2. In addition, our computational simulation provides the potential TDP-43 binding sites on TREM2. This interaction between TREM2 and TDP-43 may represent an intriguing mechanism for microglial TREM2 mediated sensing and clearance of pathological TDP-43 protein. Previous studies have shown that TDP-43 can be released by extracellular vesicles and then taken up by other neurons and contribute to neuronal damage ${ }^{62}$. It is also possible that TDP-43 can be released into the extracellular space as free cargos by unconventional protein secretion pathways which are induced by cellular stress. Indeed, we are able to detect extracellular hTDP-43 in in vitro cultures of iPSC neurons. Therefore, neuronal TDP-43 accumulation may lead 
to its release, which can be sensed and phagocytosed via microglial TREM2.

TREM2-mediated clearance mechanism is supported by structural analysis showing that neurodegenerative disease related mutations in TREM2 affects its ligand binding ability 63. Indeed, $A D$-associated mutations compromise interactions of TREM2 with $A \beta^{6}$ and GLU ${ }^{64}$. Based on our in vitro study which showed hTREM2 interacted with residue 216-414 of hTDP-43, and given the limitations of current computing technologies and the intrinsic instability of hTDP-43, we performed fragment-based MD simulations instead of MD simulations of TREM2 in complex with a full-length TDP-43 model with statistical relevance. Without any bias from disease-associated mutations in TREM2 and hTDP-43, our fragment-based MD simulations suggest that TREM2 is in contact with a potential epitope of hTDP-43 (residues 312-343). Interestingly, this epitope has the NFG fragment that abuts R47 of TREM2 and the SWG fragment that contains many mutations of the TDP-43 in ALS patients. Consistently, there are correlations among mutations in TDP-43 and/or TREM2 and the propensity of TDP-43 for aggregation ${ }^{65}$. While further studies such as in situ cross-linking are needed to biochemically confirm the TREM2 interactions with residues 312-343 of hTDP-43 in vivo, our present immunoprecipitation and MD simulation data offer new insight into the potential interaction site of TREM2 and TDP-43, highlighting that TDP-43 is an intriguing new ligand for microglial TREM2. However, we cannot ignore the fact that TDP-43 may also bind other microglia receptors. For example, previous study showed that both microglia scavenger receptor CD36 and TLR2 interact with a-synuclein ( $\alpha$ Syn) and promote microglia uptake aggregated human $\alpha$ Syn in PD model ${ }^{66}$. Moreover, TREM2 has been reported to promote microglia phagocytosis by upregulating CD36 expression ${ }^{67}$. Thus, future studies are needed to investigate whether other mediators are involved in allosteric modulation of the direct interaction between microglial TREM2 and TDP-43 or in enabling the indirect interaction between the two. In 
summary, our study found that TREM2-mediated microglial activation is an early protective response to TDP-43. The underlying mechanism may involve the interaction between TDP-43 and TREM2 that mediated phagocytosis of TDP-43 by CD11c ${ }^{+}$ microglia subpopulation. These findings point out microglial TREM2 as a potential therapeutic target for ameliorating TDP-43-related neurodegeneration.

\section{Methods}

Mice. The TREM2 knockout (KO) mouse strain was kindly provided by Dr. Marco Colonna at the Washington University School of Medicine, St. Louis. Wild-type (WT) C57BL/6J mouse strain, CX3CR $1{ }^{\mathrm{GFP} /+}$ mice and $\mathrm{rNLS} 8{ }^{22}$ were purchased from Jackson Labs. Mouse lines were housed with littermates with free access to food and water on a 12 hour light/dark cycle. All animal procedures, including husbandry, were performed under the guidelines set forth by the Mayo Clinic Institutional Animal Care and Use Committee.

Human samples. Post-mortem brain samples were dissected from the frozen brains of 16 ALS cases (age $67 \pm 9.4$ years, mean \pm s.d.) and 12 controls (age $74.7 \pm 10.6$ years) from the Brain Bank for Neurodegenerative Disorders at Mayo Clinic Jacksonville. The study was approved by Mayo Biospecimen Committee. For clinic pathological studies, cases were included only if they had good quality medical documentation and there was diagnostic concurrence of at least minimum of two neurologists. A summary of cases, including clinical and pathologic analyses, are listed in supplementary Table 2.

Cell culture and transfection. Human embryonic kidney (HEK) 293T cells were cultured in DMEM (Gibco, Thermo Fisher Scientific) supplemented with 10\% FBS (Gibco, 
Thermo Scientific). Cells were cultured at $37^{\circ} \mathrm{C}$ with $5 \% \mathrm{CO}_{2}$. HEK293 cells were co-transfected with plasmids encoding wide-type myc-human TREM2 and GFP-human TDP-43 (residues 216-414, Addgene, 28197) or GFP control by the calcium phosphate precipitation method. The expression of TREM2 or TDP-43 was assessed 24-72 $\mathrm{h}$ after infection.

Induced iPSC cultures were prepared as previously reported ${ }^{68}$. 6 -well plates were coated with hESC Qualified Geltrex (Gibco, ThermoFisher) and incubated at $37^{\circ} \mathrm{C}$ and $5 \%$ $\mathrm{CO}_{2}$ for at least $1 \mathrm{~h}$. Differentiated iPSC neurons were cultured in media composed of $\mathrm{KO}$ DMEM/F-12, 2\% B27 supplement (Gibco, ThermoFisher), $100 \mathrm{U} / \mathrm{mL}$ penicillin, $100 \mathrm{lg} / \mathrm{mL}$ streptomycin, 1\% Glutamax, $1 \mathrm{mmol} / \mathrm{L}$ dibutyryl cyclic-adenosine monophosphate (cAMP; Sigma), $5 \mathrm{lg} / \mathrm{mL}$ plasmocin (Invivogen), $1 \mathrm{mmol} / \mathrm{L}$ sodium pyruvate and $10 \mathrm{ng} / \mathrm{mL}$ each of BDNF (PreproTech), IGF1 (Corning) and GDNF (PeproTech). To express proteins in iPSC neurons, $1 \mu \mathrm{L}$ each of AAV9.CAG.hTDP-43-GFP $(9.0 \mathrm{E}+13$ viral genomes/mL; AAV9 empty vector as control) and AAV1.Tdtomato $(5.0 \mathrm{E}+12$ viral genomes $/ \mathrm{mL}$; Addgene, 59462) were added to $1 \mathrm{~mL}$ culture medium.

Intracerebroventricular injections. AAV was injected intracerebroventricularly (ICV) into C57BL/6 mouse or TREM2 KO mouse pups on postnatal day 0 (P0). ICV injections were performed as described ${ }^{69}$. Newborn mice were put onto a cold metal plate for 2-3 mins to induce hypothermic anesthesia. Cryoanesthetized neonates were injected using 10 uL Hamilton syringes affixed with a 32-gauge needle at an angle of 45 degree to a depth of $3 \mathrm{~mm}$. The injection site was located at 2/5 of the distance from the lambda suture to each eye and $2 \mathrm{uL}$ of virus $(9.0 \mathrm{E}+12$ viral genomes $/ \mathrm{mL})$ was slowly injected into each ventricle. After injection, pups were allowed to completely recover on a warming blanket and then returned to the home cage. 
Stereotaxic intracerebral injection. Adult wildtype C57BL/6 mice or TREM2 KO mice at 2 months of age were anesthetized by $2 \%$ isoflurane. $0.2 \mu \mathrm{L}$ AAV $(9.0 \mathrm{E}+12$ viral genomes $/ \mathrm{mL}$ ) was then injected into the right primary motor cortex at the following stereotaxic coordinates: $1.4 \mathrm{~mm}$ anterior to bregma, $1.5 \mathrm{~mm}$ lateral to the midline, 0.5 $\mathrm{mm}$ ventral to the dura, with bregma set at zero. The microinjections were carried out at a rate of $0.04 \mu \mathrm{L} / \mathrm{min}$, using an automated stereotaxic injection apparatus (Model UMC4, World Precision Instruments Inc.). The microsyringe was left in place for an additional 10 mins after each injection.

Immunofluorescence staining. Tissue was prepared as previously reported ${ }^{70}$. Mice were anesthetized with isoflurane $\left(5 \%\right.$ in $\left.\mathrm{O}_{2}\right)$ and then intracardially perfused with $40 \mathrm{ml}$ cold PBS, followed by $40 \mathrm{ml}$ cold $4 \%$ paraformaldehyde (PFA). Brains were removed and post-fixed in 4\% PFA for an additional 6 hours, then transferred to $30 \%$ sucrose in PBS for three days. $20 \mu \mathrm{m}$ slices were cut with a cryostat (Leica). For immunofluorescence staining, the sections were blocked for 60 mins with $10 \%$ goat serum in TBS buffer containing $0.4 \%$ Triton X-100 (Sigma), and then incubated overnight at $4{ }^{\circ} \mathrm{C}$ with a primary IgG antibody: rabbit anti-NeuN (1:500, Abcam, ab104225), rabbit anti-CD68 (1:500, Abcam, ab125212), hamster anti-CD11c (1:200, Thermo, 14011482), rabbit anti-P2Y12 (1:400, Anaspec, AS-55043A), rabbit anti-lba1 (1:500, Abcam, ab178847), rabbit anti-p-TDP-43 (1:200, Proteintech, 22309-1-AP), mouse anti-GFAP (1:500, CST, 3670), rabbit anti-CNPase (1:500, Proteintech, 13427-1-AP) or rabbit anti-TMEM119 (1:500, Abcam, ab209064). After three washes with TBST, sections were exposed to appropriate secondary antibody for 60 mins at room temperature (1:500, Alexa Fluor goat anti-rabbit 594, Thermo, a32740 or 1:500, Alexa Fluor goat anti-ArHm 647, Abcam, ab173004), washed and mounted and coverslipped with Fluoromount-G 
(SouthernBiotech). Fluorescent images were captured with a confocal microscope (LSM510, Zeiss), and cells of interest were counted and fluorescence signal intensity was quantified using ImageJ software (National Institutes of Health, Bethesda, MD).

\section{Immunohistochemistry}

Free floating brain sections $(30 \mu \mathrm{m})$ were rinsed 3 times in TBS, and incubated in $3 \%$ $\mathrm{H}_{2} \mathrm{O}_{2}$ for 30 minutes to quench endogenous peroxidase activity. IHC staining for hTDP43 antibody (mouse, 1:500, Proteintech, 60019-2-lg) and p-hTDP43 antibody (mouse, 1:500, Proteintech, 22309-1-AP) were performed using M.O.M. immunodetection kit (Vector Laboratories BMK-2202) following the manufacturer's instructions. After primary antibody (1:500), biotinylated appropriate secondary antibodies were added for 2 hours at room temperature. Vectastain-ABC Kit (Vector Laboratories) and ImmPACT DAB substrate peroxidase (HRP) kit (Vector Laboratories) were used to amplify and detect signals. Subsequently, sections were washed, mounted, and dehydrated through $70 \%$, $95 \%$, and $100 \%$ ethanol solutions. After immersing in xylene, coverslips were placed on slides using DEPEX mounting medium (Electron Microscopy Science) for observation.

NissI staining. Sample slices were incubated in 100\% ethanol for 6 mins, and then defatted in Xylene for 15 mins, followed by another 10 mins in 100\% ethanol. After rinsing in distilled water, the slides were stained with $0.5 \%$ cresyl violet acetate for 15 mins, and again rinsed in distilled water. The sections were then placed in differentiation buffer ( $0.2 \%$ acetic acid in $95 \%$ ethanol) for 2 mins, dehydrated with ethanol and Xylene, and then mounted with Depex medium. Neuronal number in the layer 5 of primary motor cortex was quantified following previous established standard criteria: (1) pyramidal neurons exhibited a characteristic triangular shape with a single large apical dendrite extending vertically towards the pial surface, (2) non-pyramidal neurons were identified 
by the absence of the preceding criteria, by the presence of two or more small calibre processes, and by their generally smaller size ${ }^{71}$.

Mass cytometry/CyTOF of isolated brain cells. Microglia antibody panel based on surface markers (supplementary Table1) was designed for brain mass cytometry/cytometry by time of flight (CyTOF). All the antibodies were provided by Mayo CyTOF core (Rochester, MN). The whole mouse brain was dissociated into a single-cell suspension using brain tissue dissociation kits following the manufacturer's instructions (Adult Brain Dissociation Kit, Miltenyi Biotec, 130-107-677). Collected cells were then incubated with metal-conjugated antibodies in cell staining buffer (ProductMaxpar Cell Staining Buffer, Fluidigm, CA). The barcoded sample was loaded onto a Helios CyTOF® system (Fluidigm) using an attached autosampler and were acquired at a rate of 200-400 events per second. Data were collected as .FCS files using the CyTOF software (Version 6.7.1014). After acquisition intrafile signal drift was normalized to the acquired calibration bead signal using the CyTOF software. Cleanup of cell debris, removal of doublets and dead cells was performed using FloJo software version 10.5.3 (Ashland, OR). Cleaned FCS files were analyzed by Cytobank (Santa Clara, CA).

Isolation of RIPA-soluble and sarkosyl-insoluble proteins. Mouse brain tissue was lysed in RIPA lysis buffer (Cell signaling), supplemented with protease inhibitors cocktail and phosphor inhibitor (Thermo Fisher Scientific), and kept on ice for 30 mins. The brain homogenate was then ultracentrifuged at $50,000 \mathrm{~g}$ for 15 mins at $4^{\circ} \mathrm{C}$. The supernatant was transferred to a new tube as RIPA-soluble protein fraction. The pellet was homogenized in $10 \%$ sucrose buffer before being ultracentrifuged at $50,000 \mathrm{~g}$ for 15 mins at $4^{\circ} \mathrm{C}$. After ultracentrifuge, the pellet was discarded and the supernatant was transferred to a new tube and incubated with sarkosyl (Sodium lauroyl sarcosinate) at a 
final concentration of $1 \%$ for 1 hour at $37^{\circ} \mathrm{C}$. Following incubation, the samples were ultracentrifuged at $50,000 \mathrm{~g}$ for 30 mins at $4^{\circ} \mathrm{C}$. The supernatant was discarded and the pellet was re-suspended to obtain the insoluble protein fraction. Soluble and insoluble fractions were then boiled in SDS-PAGE loading buffer for immunoblotting. Blots were probed with antibodies for mouse anti-hTDP-43 (1:1000, Proteintech, 60019-2-lg), rabbit anti-total TDP-43 (1:1000, Proteintech, 10782-2-AP), mouse anti-human TDP-43, phospho Ser409/410 (1:2000 , COSMOBIO, CAC-TIP-PTD-M01), rabbit anti-GFP (1:1000, Cell signaling, 2956s) and mouse anti-GAPDH (1:5000, Santa Cruz, sc-32233).

Immunoprecipitation assay. For in vitro binding assays, we transfected HEK 293T cell with myc tagged TREM2 and GFP-hTDP-43 or GFP control plasmids. Cell lysates were prepared in IP lysis buffer (50 mM Tris-HCl, pH 7.5, 0.5\% Nonidet P-40, $150 \mathrm{Mm} \mathrm{NaCl}$, 5Mm EDTA) supplemented with protease inhibitors cocktail and phosphotase inhibitor (Thermo Fisher Scientific), and kept on ice for 30 mins. The lysates were then centrifuged at $14,000 \mathrm{~g}$ for 15 mins at $4^{\circ} \mathrm{C}$. The supernatant protein concentration was measured and normalized between samples. Myc-Trap agarose beads were used to pull down the Myc-TREM2 protein following the manufacturer's instructions (Chromotek, yta-10). GFP-Trap agarose beads were used to pull down the GFP-human TDP-43 (residues 216-414) or GFP protein (Chromotek, gta-10). Blots were probed with antibodies for mouse anti-Myc antibody (1:1000, Sigma, SAB4700447), rabbit anti-TDP-43 (1:1000, Proteintech, 10782-2-AP), rabbit anti-GFP (1:1000, Cell signaling, 2956s).

For in vivo binding assays, TDP-43/TREM2 interaction was detected in rNLS8 mouse with 4 weeks DOX off and 2 weeks DOX on. Mouse TREM2 was immunoprecipitated from 400-500 ug protein per sample using 1.5 ug mouse TREM2 biotinylated antibody 
(R\&D, BAF1729). After overnight incubation at $4^{\circ} \mathrm{C}$, biotinylated antibody was pulled down using NeutrAvidin Agarose beads (Thermo Fisher 29200) for at least $2 \mathrm{~h}$ at $4^{\circ} \mathrm{C}$. Avidin beads were collected by centrifugation at 2,000 $\times \mathrm{g}$ for 3 mins and washed four times with IP lysis buffer, and then boiled in SDS-PAGE loading buffer for immunoblotting. Blots were probed with antibodies for rat anti-mouse TREM2 antibody [5F4] (1:500, abcam, ab252876) and mouse anti-hTDP-43 (1:1000, Proteintech, 60019-2-lg).

Endogenous TDP-43/TREM2 interactions were also determined using frozen autopsied specimens of ALS patients' spinal cord. Lysates containing 400-500 $\mu \mathrm{g}$ protein were incubated with rabbit anti-human TREM2 antibody (Cell Signaling, 91068) / Dynabeads protein $\mathrm{A}$ (Thermo fisher, 22810) at $4^{\circ} \mathrm{C}$ overnight. Protein $\mathrm{A}$ beads were collected by centrifugation at 2,000 $\times \mathrm{g}$ for 3 mins and washed four times with IP lysis buffer, and then boiled in SDS-PAGE loading buffer for immunoblotting. Blots were probed with antibodies for goat anti-human TREM2 antibody, (1:1000, R\&D, AF1828) and mouse anti-h-TDP-43 (1:1000, Proteintech, 60019-2-lg).

Immunoblotting. Mouse brain tissue or human tissue samples were lysed in RIPA lysis buffer (Cell signaling), supplemented with protease inhibitors cocktail and phosphatase inhibitor (Thermo Fisher Scientific), and kept on ice for 30 mins. The lysates were then centrifuged at $14,000 \times \mathrm{g}$ for $30 \mathrm{mins}$ at $4^{\circ} \mathrm{C}$. The supernatant protein concentration was measured and normalized between samples, and was boiled in SDS loading buffer. Prepared samples were subjected to $4 \%-20 \%$ SDS-PAGE (Bio-Rad laboratories).

Liquid Chromatography with tandem mass spectrometry (LC-MS/MS) and affinity purification coupled with mass spectrometry (AP-MS) interactome analysis. To detect interacting proteins of TREM2, HEK 293 cells or rNLS mice brains were lysed in 
lysis buffer (50 mM Tris-HCl, protease inhibitors cocktail (Thermo Fisher Scientific), phosphatase inhibitor (Thermo Fisher Scientific), and 0.002\% zwittergent 3-16) and kept on ice for additional 30 mins. After centrifugation, the supernatant were incubated with rabbit anti-human TREM2 antibody (Cell Signaling, 91068) or anti-mouse TREM2 antibody on a rotator, respectively. After 3 washes, immunoprecipitated proteins were eluted by boiling in Lammaeli buffer for immunoblotting. Alternatively, immunoprecipitated proteins were eluted by boiling in $1 \%$ SDS, resolved by non-reducing gel and subjected to reduction, alkylation and trypsin digestion using an in-gel digestion protocol. The extracted tryptic peptides were dried in a speed vac. The dried peptides were reconstituted in sample loading buffer $(0.2 \%$ formic acid, $0.1 \%$ TFA, $0.002 \%$ zwittergent 3-16) and analyzed by nano-ESI-LC/MS/MS with a $Q$ Exactive mass spectrometer coupled to a dionex nano-LC system (Thermo Scientific; Waltham, MA) with a $32 \mathrm{~cm}, 100 / 15$ um, Acclaim 2.2 um column. The LC system used a gradient with solvent $\mathrm{A}(2 \% \mathrm{ACN}, 0.2 \%$ formic acid, in water) and solvent $\mathrm{B}(80 \% \mathrm{ACN}, 10 \%$ IPA, $0.2 \%$ formic acid, in water) with a $400 \mathrm{~nL} / \mathrm{min}$ flow rate as follows: $4-5 \mathrm{~min} 5 \%$ B; 5 - 95 min 5-45 \% linear gradient; 95-98 min 45-95\% linear gradient; 98-102 min 95\% B; 102-104 $\min 95-10 \%$ B linear gradient; $104-107$ min 10\% B; 107-115 min 10-95\% B; 115-118min 95\% B; 118-121 min 95-5\% B linear gradient; 121-127 min 5\% B. Mass spectrometer was operated in data-dependent mode with a MS survey scan $(350-1800 \mathrm{~m} / \mathrm{z})$ in Orbitrap (70,000 resolution $200 \mathrm{~m} / \mathrm{z}, 3 \times 106$ AGC target and $100 \mathrm{~ms}$ maximal ion time) and $20 \mathrm{MS} / \mathrm{MS}$ fragments scans (17,500 resolution, $2 \times 105$ AGC target, 50 ms maximal ion time, 28 normalized collision energy, $2.5 \mathrm{~m} / \mathrm{z}$ isolation window) with a dynamic range set to 25 seconds. The tandem mass spectra were searched using SEQUEST ${ }^{72}$ search algorithms against a Uniprot database (human 2019_07 release for 293T ColP and mouse 2019_08 release supplemented with human TDP-43 protein sequence for mouse CoIP) through the Proteome Discoverer platform (version 2.5, Thermo Scientific). The 
search parameters included a maximum of two missed cleavages; carbamidomethylation at cysteine as a fixed modification; N-terminal acetylation, deamidation at asparagine and glutamine, oxidation at methionine as variable modifications; the monoisotopic peptide tolerance, $5 \mathrm{ppm}$ and MS/MS tolerance, $0.02 \mathrm{Da}$. The target-decoy analysis was used for validation. The false discovery rate (FDR) was set to $1 \%$ at both peptide and protein level.

Hindlimb clasping. To examine hindlimb clasping, mice were gently removed from their home cage and suspended by the tail for $10 \mathrm{~s}$ and provided a hindlimb clasping score. If the mouse exhibits normal escape extension without limb clasping, it was assigned a score of 4 . If one hindlimb exhibits incomplete splay and loss of mobility and toes exhibit normal splay, it received a score of 3 . If both hindlimbs exhibit incomplete splay and loss of mobility and toes exhibit normal splay, it received a score of 2 . If both hindlimbs exhibit clasping with curled toes and immobility, it received a score of 1 . If forelimbs and hindlimbs exhibit clasping and are crossed, curled toes and immobility, it received a score of 0 .

Open field testing. Locomotor activity of mice was assessed in sound-insulated, rectangular activity chambers with continually running fans, infrared lasers, and sensors (Med Associates, St Albans, VT, USA). Prior to testing, mice were acclimatized to the room for $1 \mathrm{~h}$. Mice were habituated for 5 mins in the Open Field chamber (without recording) then placed back to home cage for another 5 mins. Afterwards, mice were introduced back to the chambers and activities were monitored for 30 mins. Locomotor function was quantified by the total distance mice travelled in the chamber which was recorded on a computer with Med-PC software Version 4.0. 
Rotarod. The Rotarod performance test evaluates mouse balance and motor coordination. Mice were brought to the test room $1 \mathrm{~h}$ before testing. Rotarod tests were performed using a five-lane Rotarod apparatus (Med Associates Inc), started at 4 revolutions per minute and steadily accelerated to 40 revolutions per minute over a 5 mins period. Each mouse was tested 3 times at 10-min intervals.

Molecular dynamics (MD) simulations. The LCD fragment was manually docked into the paratope region of the crystal structure of the ECD (Residues 21-219) of human TREM2 (PDB ID: 6B8O) as described above. The resulting complex was neutralized with counterions and then energy-minimized according to a protocol described below. In this complex, all ionic residues were treated as AMBER residues of ASP, GLU, ARG and LYS, all histidine residues were treated as HIE, and all cysteine residues were treated as CYX. The topology and coordinate files of the complex were generated by the tLeAP module of AmberTools 13 (University of California, San Francisco). The energy minimization and MD simulations were performed using SANDER and PMEMD of AMBER 16 (University of California, San Francisco) with force field FF12MC ${ }^{32}$. The energy minimization used dielectric constant of 1.0 and 100 cycles of steepest-descent minimization followed by 900 cycles of conjugate-gradient minimization. The energy-minimized model was solvated with the TIP3P water using "solvate box $\mathrm{m}$ TIP3PBOX 8.2" and energy minimized for 100 cycles of steepest-descent minimization followed by 900 cycles of conjugate-gradient minimization using the SANDER module ${ }^{73}$. The resulting system was heated from $5 \mathrm{~K}$ to $320 \mathrm{~K}$ at a rate of $10 \mathrm{~K} / \mathrm{ps}$ under constant temperature and constant volume, equilibrated for $10^{6}$ time steps under constant temperature of $320 \mathrm{~K}$ and constant pressure of 1 atm employing isotropic molecule-based scaling, and finally simulated, without any harmonic restraint, in 20 316-ns, distinct, independent, unrestricted, unbiased, and isobaric-isothermal MD 
simulations with FF12MC using the PMEMD module of AMBER 16 with a periodic boundary condition at $320 \mathrm{~K}$ and $1 \mathrm{~atm}$. The 20 unique seed numbers for initial velocities of Simulations 1-20 were taken from Ref. ${ }^{74}$. All simulations used (i) a dielectric constant of 1.0, (ii) Langevin thermostat ${ }^{75}$ with a collision frequency of $2 \mathrm{ps}^{-1}$, (iii) the Particle Mesh Ewald method to calculate electrostatic interactions of two atoms at a separation of $>8 \AA^{76}$, (iv) $\Delta t=1.00 \mathrm{fs}$ of the standard-mass time ${ }^{32,77}$, (v) the SHAKE-bond-length constraints applied to all bonds involving hydrogen, (vi) a protocol to save the image closest to the middle of the "primary box" to the restart and trajectory files, (vii) the revised alkali and halide ions parameters ${ }^{78}$, (viii) a cutoff of $8.0 \AA$ for nonbonded interactions, (ix) the atomic masses of the entire simulation system (both solute and solvent) that were reduced uniformly by tenfold ${ }^{32,77}$, and $(x)$ default values of all other inputs of the PMEMD module. The FF12MC parameters are available in the Supporting Information of previous literature ${ }^{77}$. The complex conformations were saved at $100-p s$ intervals in all simulations. The cluster analysis of all saved complex conformations was performed using the CPPTRAJ module of AmberTools 16 (University of California, San Francisco) with the average-linkage algorithm ${ }^{89}$ (epsilon $=2.0 \AA$; RMS on all alpha carbon atoms of Residues 32-51, 60-97, and 108-124 of TREM2 and the entire LCD fragment). Centering the coordinates of the complex and imaging the coordinates to the primary unit cell were performed prior to the cluster analysis. The most populated conformation of the MD simulations identified by the cluster analysis was used as the simulation-refined model of the complex shown in Fig. 7.

Statistics. Statistical details of the experiments, including sample sizes and statistical tests are described in figure legends. Results are displayed as mean \pm SEM. All statistical analysis was performed with GraphPad Prism software (version 8). Samples sizes are comparable to similar studies ${ }^{25,37,79}$. No statistical methods were used to 
predetermine sample sizes. All data were normally distributed and standard deviations were calculated to determine whether the data met assumptions of the statistical approach. Mean values for multiple groups was compared using a two-way ANOVA followed by Tukey's post hoc test. Comparison of two groups was performed using a two-tailed unpaired Student's $t$-test. $P$ values $<0.05$ were considered as statistically significant.

\section{Data availability}

Further information and requests for resources and reagents should be directed to and will be fulfilled by the lead contact, Dr. Long-Jun Wu (wu.longjun@mayo.edu).

\section{Acknowledgments}

The authors thank Dr. Marco Colonna (Washington University) for providing TREM2 KO mice; Dr. Ronald L. Klein (Louisiana State University) for providing AAV virus, Dr. Vanda A. Lennon (Mayo Clinic Rochester) for thoughtful discussion and manuscript editing; Dr. Wilfried Rossoll (Mayo Clinic Florida) for insightful discussion and suggestions; Dr. Aaron J. Johnson and Dr. Kevin D. Pavelko (Mayo Clinic Rochester Immune Monitoring Core) for assistance with CyTOF sample preparation and data analysis; Dr. Akhilesh Pandey and Dr. Jun Zhong (Mayo Clinic Rochester Proteomics Core) for assistance with LC-MS/MS and data analysis; Dr. Charles Howe and Dr. Benjamin Clarkson (Mayo Clinic Rochester) for providing iPSC-derived neurons; Dr. Richard M Weinshilboum (Mayo Clinic Rochester) for assistance with biochemistry experiments. The current study is supported by NIH grants R21AG064159 and R01NS088627 to L.J.W and R01AG066395 to G.B and N.Z. 


\section{Author contributions}

M.X., Y.U.L. and L.-J.W. designed the experiments and wrote the manuscript; M.X. and Y.U.L. performed most of the experiments; S.Z., Y.L., L.Z., U.S., Y.Z., N.Z., C.C.L performed some of the experiments; M.X. and D.B.B. performed data analysis; Y.-P.P. designed, performed, and analyzed the MD simulation study; Y.A.M., L.W., D.W.D., M.P.M. and G.B. provided resources for specific experiments.

\section{Competing interests}

The authors declare no competing interests.

\section{References}

1. Hickman, S., Izzy, S., Sen, P., Morsett, L. \& El Khoury, J. Microglia in neurodegeneration. Nat Neurosci 21, 1359-1369 (2018).

2. Ulland, T.K. \& Colonna, M. TREM2 - a key player in microglial biology and Alzheimer disease. Nat Rev Neurol 14, 667-675 (2018).

3. Guerreiro, R., et al. TREM2 variants in Alzheimer's disease. N Engl J Med 368, 117-127 (2013).

4. Jonsson, T., et al. Variant of TREM2 associated with the risk of Alzheimer's disease. N Engl J Med 368, 107-116 (2013).

5. Wang, Y., et al. TREM2 lipid sensing sustains the microglial response in an Alzheimer's disease model. Cell 160, 1061-1071 (2015).

6. Zhao, Y., et al. TREM2 Is a Receptor for beta-Amyloid that Mediates Microglial Function. Neuron 97, 1023-1031 e1027 (2018).

7. Ou, S.H., Wu, F., Harrich, D., Garcia-Martinez, L.F. \& Gaynor, R.B. Cloning and characterization of a novel cellular protein, TDP-43, that binds to human immunodeficiency virus type 1 TAR DNA sequence motifs. J Virol 69, 3584-3596 (1995).

8. Neumann, M., et al. Ubiquitinated TDP-43 in frontotemporal lobar degeneration and amyotrophic lateral sclerosis. Science 314, 130-133 (2006). 
9. Cairns, N.J., et al. TDP-43 in familial and sporadic frontotemporal lobar degeneration with ubiquitin inclusions. Am J Pathol 171, 227-240 (2007).

10. Cady, J., et al. TREM2 variant p.R47H as a risk factor for sporadic amyotrophic lateral sclerosis. JAMA Neurol 71, 449-453 (2014).

11. Rayaprolu, S., et al. TREM2 in neurodegeneration: evidence for association of the p.R47H variant with frontotemporal dementia and Parkinson's disease. Mol Neurodegener 8, 19 (2013).

12. Krasemann, S., et al. The TREM2-APOE Pathway Drives the Transcriptional Phenotype of Dysfunctional Microglia in Neurodegenerative Diseases. Immunity 47, 566-581 e569 (2017).

13. Maniatis, S., et al. Spatiotemporal dynamics of molecular pathology in amyotrophic lateral sclerosis. Science 364, 89-93 (2019).

14. Cooper-Knock, J., et al. A data-driven approach links microglia to pathology and prognosis in amyotrophic lateral sclerosis. Acta Neuropathol Commun 5, 23 (2017).

15. Walker, A.K., et al. Functional recovery in new mouse models of ALS/FTLD after clearance of pathological cytoplasmic TDP-43. Acta Neuropathol 130, 643-660 (2015).

16. Wang, X., et al. Early intervention of tau pathology prevents behavioral changes in the rTg4510 mouse model of tauopathy. PLoS One 13, e0195486 (2018).

17. Furukawa, Y., Kaneko, K., Watanabe, S., Yamanaka, K. \& Nukina, N. A seeding reaction recapitulates intracellular formation of Sarkosyl-insoluble transactivation response element (TAR) DNA-binding protein-43 inclusions. J Biol Chem 286, 18664-18672 (2011).

18. Hasegawa, M., et al. Phosphorylated TDP-43 in frontotemporal lobar degeneration and amyotrophic lateral sclerosis. Ann Neurol 64, 60-70 (2008).

19. Yousef, A., et al. Neuron loss and degeneration in the progression of TDP-43 in frontotemporal lobar degeneration. Acta Neuropathol Commun 5, 68 (2017).

20. Perry, V.H., Nicoll, J.A. \& Holmes, C. Microglia in neurodegenerative disease. Nat Rev Neurol 6, 193-201 (2010).

21. Mrdjen, D., et al. High-Dimensional Single-Cell Mapping of Central Nervous System Immune Cells Reveals Distinct Myeloid Subsets in Health, Aging, and Disease. Immunity 48, 380-395 e386 (2018).

22. Jung, S., et al. Analysis of fractalkine receptor CX(3)CR1 function by targeted deletion and green fluorescent protein reporter gene insertion. Mol Cell Biol 20, 4106-4114 (2000).

23. Kamphuis, W., Kooijman, L., Schetters, S., Orre, M. \& Hol, E.M. Transcriptional profiling of CD11c-positive microglia accumulating around amyloid plaques in a mouse model for Alzheimer's disease. Biochim Biophys Acta 1862, 1847-1860 
(2016).

24. Eyo, U.B., et al. P2Y12R-Dependent Translocation Mechanisms Gate the Changing Microglial Landscape. Cell Rep 23, 959-966 (2018).

25. Spiller, K.J., et al. Microglia-mediated recovery from ALS-relevant motor neuron degeneration in a mouse model of TDP-43 proteinopathy. Nat Neurosci 21, 329-340 (2018).

26. Takahashi, K., Rochford, C.D. \& Neumann, H. Clearance of apoptotic neurons without inflammation by microglial triggering receptor expressed on myeloid cells-2. J Exp Med 201, 647-657 (2005).

27. Hsieh, C.L., et al. A role for TREM2 ligands in the phagocytosis of apoptotic neuronal cells by microglia. J Neurochem 109, 1144-1156 (2009).

28. Steinacker, P., et al. TDP-43 in cerebrospinal fluid of patients with frontotemporal lobar degeneration and amyotrophic lateral sclerosis. Arch Neurol 65, 1481-1487 (2008).

29. Sproviero, D., et al. Pathological Proteins Are Transported by Extracellular Vesicles of Sporadic Amyotrophic Lateral Sclerosis Patients. Front Neurosci 12, 487 (2018).

30. Francois-Moutal, L., et al. Structural Insights Into TDP-43 and Effects of Post-translational Modifications. Front Mol Neurosci 12, 301 (2019).

31. Arnold, E.S., et al. ALS-linked TDP-43 mutations produce aberrant RNA splicing and adult-onset motor neuron disease without aggregation or loss of nuclear TDP-43. Proc Natl Acad Sci U S A 110, E736-745 (2013).

32. Pang, Y.P. FF12MC: A revised AMBER forcefield and new protein simulation protocol. Proteins 84, 1490-1516 (2016).

33. Guenther, E.L., et al. Atomic structures of TDP-43 LCD segments and insights into reversible or pathogenic aggregation. Nat Struct Mol Biol 25, 463-471 (2018).

34. Hardiman, O., et al. Amyotrophic lateral sclerosis. Nat Rev Dis Primers 3, 17071 (2017).

35. Li, Q., et al. Developmental Heterogeneity of Microglia and Brain Myeloid Cells Revealed by Deep Single-Cell RNA Sequencing. Neuron 101, 207-223 e210 (2019).

36. Liu, Y.U., et al. Neuronal network activity controls microglial process surveillance in awake mice via norepinephrine signaling. Nat Neurosci 22, 1771-1781 (2019).

37. Parhizkar, S., et al. Loss of TREM2 function increases amyloid seeding but reduces plaque-associated ApoE. Nat Neurosci 22, 191-204 (2019).

38. Leyns, C.E.G., et al. TREM2 function impedes tau seeding in neuritic plaques. Nat Neurosci 22, 1217-1222 (2019).

39. Lee, C.Y.D., et al. Elevated TREM2 Gene Dosage Reprograms Microglia Responsivity and Ameliorates Pathological Phenotypes in Alzheimer's Disease 
Models. Neuron 97, 1032-1048 e1035 (2018).

40. Konishi, H. \& Kiyama, H. Microglial TREM2/DAP12 Signaling: A Double-Edged Sword in Neural Diseases. Front Cell Neurosci 12, 206 (2018).

41. Mazaheri, F., et al. TREM2 deficiency impairs chemotaxis and microglial responses to neuronal injury. EMBO Rep 18, 1186-1198 (2017).

42. Zheng, $\mathrm{H}$., et al. TREM2 Promotes Microglial Survival by Activating Wnt/beta-Catenin Pathway. J Neurosci 37, 1772-1784 (2017).

43. Guan, Z., et al. Injured sensory neuron-derived CSF1 induces microglial proliferation and DAP12-dependent pain. Nat Neurosci 19, 94-101 (2016).

44. Gu, N., et al. Spinal Microgliosis Due to Resident Microglial Proliferation Is Required for Pain Hypersensitivity after Peripheral Nerve Injury. Cell Rep 16, 605-614 (2016).

45. Otero, K., et al. Macrophage colony-stimulating factor induces the proliferation and survival of macrophages via a pathway involving DAP12 and beta-catenin. Nat Immunol 10, 734-743 (2009).

46. Maes, M.E., Colombo, G., Schulz, R. \& Siegert, S. Targeting microglia with lentivirus and AAV: Recent advances and remaining challenges. Neurosci Lett 707, 134310 (2019).

47. Sierra, A., Abiega, O., Shahraz, A. \& Neumann, H. Janus-faced microglia: beneficial and detrimental consequences of microglial phagocytosis. Front Cell Neurosci 7, 6 (2013).

48. Brown, G.C. \& Neher, J.J. Microglial phagocytosis of live neurons. Nat Rev Neurosci 15, 209-216 (2014).

49. Fu, R., Shen, Q., Xu, P., Luo, J.J. \& Tang, Y. Phagocytosis of microglia in the central nervous system diseases. Mol Neurobiol 49, 1422-1434 (2014).

50. Hong, S., et al. Complement and microglia mediate early synapse loss in Alzheimer mouse models. Science 352, 712-716 (2016).

51. Koizumi, S., et al. UDP acting at P2Y6 receptors is a mediator of microglial phagocytosis. Nature 446, 1091-1095 (2007).

52. Tufail, Y., et al. Phosphatidylserine Exposure Controls Viral Innate Immune Responses by Microglia. Neuron 93, 574-586 e578 (2017).

53. Gottfried-Blackmore, A., et al. Acute in vivo exposure to interferon-gamma enables resident brain dendritic cells to become effective antigen presenting cells. Proc Natl Acad Sci U S A 106, 20918-20923 (2009).

54. Das, R. \& Chinnathambi, S. Microglial priming of antigen presentation and adaptive stimulation in Alzheimer's disease. Cell Mol Life Sci 76, 3681-3694 (2019).

55. Harms, A.S., et al. MHCII is required for alpha-synuclein-induced activation of microglia, CD4 $\mathrm{T}$ cell proliferation, and dopaminergic neurodegeneration. $J$ 
Neurosci 33, 9592-9600 (2013).

56. Bulloch, K., et al. CD11c/EYFP transgene illuminates a discrete network of dendritic cells within the embryonic, neonatal, adult, and injured mouse brain. $J$ Comp Neurol 508, 687-710 (2008).

57. Sheean, R.K., et al. Association of Regulatory T-Cell Expansion With Progression of Amyotrophic Lateral Sclerosis: A Study of Humans and a Transgenic Mouse Model. JAMA Neurol 75, 681-689 (2018).

58. Brochard, V., et al. Infiltration of CD4+ lymphocytes into the brain contributes to neurodegeneration in a mouse model of Parkinson disease. J Clin Invest 119, 182-192 (2009).

59. Thonhoff, J.R., et al. Expanded autologous regulatory T-lymphocyte infusions in ALS: A phase I, first-in-human study. Neurol Neuroimmunol Neuroinflamm 5, e465 (2018).

60. Sato-Hashimoto, M., et al. Microglial SIRPalpha regulates the emergence of $\mathrm{CD} 11 \mathrm{c}(+)$ microglia and demyelination damage in white matter. Elife 8(2019).

61. Atagi, Y., et al. Apolipoprotein E Is a Ligand for Triggering Receptor Expressed on Myeloid Cells 2 (TREM2). J Biol Chem 290, 26043-26050 (2015).

62. Feiler, M.S., et al. TDP-43 is intercellularly transmitted across axon terminals. $J$ Cell Biol 211, 897-911 (2015).

63. Kober, D.L., et al. Neurodegenerative disease mutations in TREM2 reveal a functional surface and distinct loss-of-function mechanisms. Elife 5(2016).

64. Yeh, F.L., Wang, Y., Tom, I., Gonzalez, L.C. \& Sheng, M. TREM2 Binds to Apolipoproteins, Including APOE and CLU/APOJ, and Thereby Facilitates Uptake of Amyloid-Beta by Microglia. Neuron 91, 328-340 (2016).

65. Ling, J.P., Pletnikova, O., Troncoso, J.C. \& Wong, P.C. TDP-43 repression of nonconserved cryptic exons is compromised in ALS-FTD. Science 349, 650-655 (2015).

66. Panicker, N., et al. Fyn kinase regulates misfolded alpha-synuclein uptake and NLRP3 inflammasome activation in microglia. J Exp Med 216, 1411-1430 (2019).

67. Kim, S.M., et al. TREM2 promotes Abeta phagocytosis by upregulating C/EBPalpha-dependent CD36 expression in microglia. Sci Rep 7, 11118 (2017).

68. Clarkson, B.D.S., Patel, M.S., LaFrance-Corey, R.G. \& Howe, C.L. Retrograde interferon-gamma signaling induces major histocompatibility class I expression in human-induced pluripotent stem cell-derived neurons. Ann Clin Transl Neurol 5, 172-185 (2018).

69. Kim, J.Y., Grunke, S.D., Levites, Y., Golde, T.E. \& Jankowsky, J.L. Intracerebroventricular viral injection of the neonatal mouse brain for persistent and widespread neuronal transduction. J Vis Exp, 51863 (2014).

70. Peng, J., et al. Microglia and monocytes synergistically promote the transition 
from acute to chronic pain after nerve injury. Nat Commun 7, 12029 (2016).

71. Gittins, R. \& Harrison, P.J. Neuronal density, size and shape in the human anterior cingulate cortex: a comparison of Nissl and NeuN staining. Brain Res Bull 63, 155-160 (2004).

72. Eng, J.K., McCormack, A.L. \& Yates, J.R. An approach to correlate tandem mass spectral data of peptides with amino acid sequences in a protein database. $J \mathrm{Am}$ Soc Mass Spectrom 5, 976-989 (1994).

73. Jorgensen, W.L., Chandreskhar, J., Madura, J.D., Impey, R.W. \& Klein, M.L. Comparison of simple potential functions for simulating liquid water. J. Chem. Phys. 79, 926-935 (1983).

74. Pang, Y.-P. Use of 1-4 interaction scaling factors to control the conformational equilibrium between $\alpha$-helix and $\beta$-strand. Biochem. Biophys. Res. Commun. 457, 183-186 (2015).

75. Larini, L., Mannella, R. \& Leporini, D. Langevin stabilization of molecular-dynamics simulations of polymers by means of quasisymplectic algorithms. J Chem Phys 126, 104101 (2007).

76. Darden, T.A., York, D.M. \& Pedersen, L.G. Particle mesh Ewald: An N log(N) method for Ewald sums in large systems. J. Chem. Phys. 98, 10089-10092 (1993).

77. Pang, Y.P. Low-mass molecular dynamics simulation for configurational sampling enhancement: More evidence and theoretical explanation. Biochem Biophys Rep 4, 126-133 (2015).

78. Joung, I.S. \& Cheatham, T.E. Determination of alkali and halide monovalent ion parameters for use in explicitly solvated biomolecular simulations. J. Phys. Chem. B 112, 9020-9041 (2008).

79. Leyns, C.E.G., et al. TREM2 function impedes tau seeding in neuritic plaques. Nat Neurosci (2019). 
Figure legends

Fig 1. TREM2 deficiency aggravates hTDP-43-induced behavioral deficits and neurodegeneration.

GFP tagged hTDP-43 protein (GFP-hTDP-43) expression was induced via intracerebroventricular injection of AAV9.CAG.hTDP43-GFP in neonatal mice (AAV9.CAG.GFP as control). a, Study design and timeline for the neonatal ICV injection model. b, Representative images of GFP-hTDP-43 expression in both nucleus and cytosol of neurons in the primary motor cortex of WT mice at 35 days post-infection (dpi); Scale bar, $10 \mu \mathrm{m}$. c-d, Representative images of hTDP-43 (c) or p-hTDP-43 (d) expression in the motor cortex at 35 dpi after AAV-control or AAV-hTDP-43 infection; Scale bar, $20 \mu \mathrm{m}$. e, Representative images of typical clasping phenotype in WT mice expressing hTDP-43 at 14 dpi. f, Kaplan-Meier survival curves show the percentage of mice alive at each postnatal day up to $60 \mathrm{dpi}(\mathrm{n}=20$ per group). $\mathbf{g}$, Hindlimb clasping response scores collected over 70 days ( $n=10$ per group). $h$, Average latency to fall

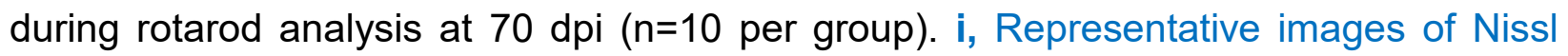
staining in the primary motor cortex of indicated groups at $35 \mathrm{dpi}$; Dashed lines indicate the borders of layer 4\&5; Scale bar, $100 \mu \mathrm{m}$; High magnification images as indicated by area in dotted white box showing neuronal morphology of the cortical layer $\mathrm{V}$ at the bottom; Scale bar, $50 \mu \mathrm{m}$. Red arrows indicate neuronal shrinkage and red arrowheads indicate a significant loss of Nissl staining. j, Quantification of neuron numbers in the primary motor cortex of indicated groups at $35 \mathrm{dpi}$ ( $\mathrm{n}=10$ per group). Data represented as

mean \pm SEM. Significance was calculated using two-way ANOVA followed by Tukey's post hoc test; $n$.s., not significant; ${ }^{*} P<0.05,{ }^{* *} P<0.01,{ }^{* * *} P<0.001$. 
Fig 2. TREM2 deficiency abolishes hTDP-43-induced $\mathrm{CD}_{11 \mathrm{c}^{+}}$microglia subpopulation.

hTDP-43 protein expression was induced via intracerebroventricular injection of AAV9.CAG.hTDP-43 in neonatal mice (AAV9.CAG.Empty as control). a, Schematic workflow for cytometry by time of flight mass spectrometry (CyTOF) and immunostaining. b, t-SNE map displaying $C D 45^{+} \mathrm{CD} 11 \mathrm{~b}^{+}$sampled cells from the brain of steady-state 6 to 8-week-old WT mice analyzed by mass cytometry. Colors correspond to FlowSOM-guided clustering of cell populations $(n=8$, representative of 4 independent experiments). c, Spanning-tree progression analysis of density-normalized events (SPADE) on $C D 45^{+} \mathrm{CD} 11 \mathrm{~b}^{+}$cell populations. The size of each spot is determined by the number of cells within this population. d, Microglia were plotted onto a t-SNE. Plots represent distinguishing cell surface markers for microglia of 6-8 week-old WT mice. Clustering analysis revealed a major microglia population characterized by

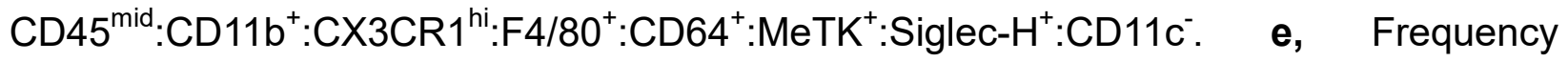
analysis of microglia based on manual gating of indicated groups at $35 \mathrm{dpi}(n=8$, representative of 4 independent experiments). Increased number of microglia was observed in both WT and TREM2 KO mice expressing hTDP-43. f, SPADE reveals a unique CD11 ${ }^{+}$microglia sub-population in WT mice expressing hTDP-43 at $35 \mathrm{dpi}$. In hTDP expressing TREM2 KO mice, $\mathrm{CD}_{11 \mathrm{c}^{+}}$microglia sub-population was largely abolished. The size of each spot is determined by the number of cells within this population. The color code shows the expression level of CD11c. g, Representative images of CD11c (purple) expression by immunostaining in the primary motor cortex of indicated groups at $35 \mathrm{dpi}$; Scale bar, $100 \mu \mathrm{m}$. High magnification images as indicated by area in dotted white box showing the single microglia at the bottom; Scale bar, $10 \mu \mathrm{m} . \mathbf{h}$, Average frequency of microglia expressing CD11c in the primary motor cortex of indicated groups at $35 \mathrm{dpi}(\mathrm{n}=8$ per group). CD11c microglia subpopulation was present 
in the WT mice expressing hTDP-43; however, few in TREM2 KO mice expressing hTDP-43 were observed. Data represented as mean \pm SEM. Significance was calculated using two-way ANOVA followed by Tukey's post hoc test; n.s., not significant; ${ }^{*} P<0.05$, ${ }^{* *} P<0.01,{ }^{* * *} P<0.001$.

Fig 3. TREM2 deficiency locks microglia into a homeostatic state in TDP-43-induced neurodegeneration.

In adult local injection model, hTDP-43 or GFP-hTDP-43 was expressed in the primary motor cortex of 2-month-old mice via stereotactic intracerebral injection of AAV9.CAG.hTDP-43 or AAV9.CAG.hTDP43-GFP (AAV9.CAG.Empty or AAV9.CAG.GFP as control). a, Study design and timeline for local hTDP-43 expression model. b, Representative images (upper panels; Scale bar, $100 \mu \mathrm{m}$ ) and transformed skeletal images (lower panels; Scale bar, $10 \mu \mathrm{m}$ ) of GFP-expressing microglia in the primary motor cortex of indicated groups at $14 \mathrm{dpi}$. c-e, Quantification of GFP-expressing microglia number of branches (c, n=10 per group), soma size (d, n=15 per group) and process length (e, n=10 per group) in the primary motor cortex of indicated groups at 14 dpi. WT microglia displayed a reactive phenotype as reduced branches, increased soma size and shorter processes in response to hTDP-43 expression. However, this hTDP-43-induced reactive phenotype was significantly attenuated in TREM2 KO mice. f, Representative images of P2Y12 expression (red) in the primary motor cortex of indicated groups at $7 \mathrm{dpi}$; Scale bar, $100 \mu \mathrm{m}$. g, Representative images of TMEM119 expression (red) in the primary motor cortex of indicated groups at $7 \mathrm{dpi}$; Scale bar, 100 $\mu \mathrm{m} . \mathbf{h}, \mathbf{i}$, Quantification of relative P2Y12 and TMEM119 positive area in the primary motor cortex of indicated groups at $7 \mathrm{dpi}$ ( $\mathrm{n}=10$ per group). j, Representative images of CD11c (purple), hTDP-43 (green), and DAPI staining (blue) in the primary motor cortex of indicated groups at $28 \mathrm{dpi}$. Scale bar, $100 \mu \mathrm{m}$. High magnification images as indicated 
by area in dotted white box showing on the right; Scale bar, $10 \mu \mathrm{m}$. k. Analysis of co-localization of CD11c (purple curves) with GFP-hTDP-43 (green curves) in the primary motor cortex of indicated groups at $28 \mathrm{dpi}$. Fluorescence intensity profiles of CD11c and GFP-hTDP-43 show the distribution of fluorescence across the yellow dotted arrows in (j). I, Average frequency of hTDP-43 associated CD11c ${ }^{+}$microglia in the primary motor cortex of indicated groups at $28 \mathrm{dpi}(\mathrm{n}=7$ per group). Results indicate that CD11 $\mathrm{c}^{+}$microglia subpopulation was present in WT mice upon hTDP-43 expression. However, $\mathrm{CD} 11 \mathrm{c}^{+}$microglia were not observed in TREM2 KO mice expressing GFP-hTDP-43. Data are represented as mean \pm SEM. Significances were calculated using Two-way ANOVA, Tukey's post-hoc analysis (c-e, $\mathbf{h}$ and $\mathbf{i}$ ) and student $\mathbf{t}$ test (I); n.s., not significant; ${ }^{\star} P<0.05,{ }^{* *} P<0.01,{ }^{* * *} P<0.001$.

Fig 4. TREM2 deficiency impairs microglial phagocytosis of pathological hTDP-43 protein.

hTDP-43 or GFP-hTDP-43 was expressed in the primary motor cortex of 2-month-old mice via stereotactic intracerebral injection of AAV9.CAG.hTDP-43 or AAV9.CAG.hTDP43-GFP (AAV9.CAG.Empty or AAV9.CAG.GFP as control). a, Representative images of CD68 expression (red) in microglia (CX3CR1-GFP, green) in the primary motor cortex of indicated groups at $14 \mathrm{dpi}$; Scale bar, $100 \mu \mathrm{m}$. b, Quantification of relative CD68 positive area in the primary motor cortex of indicated groups at 14 dpi ( $n=5$ per group). Results show hTDP-43 induced increase of CD68 expression in WT but not in TREM2 KO mice. c, Representative images of CD68 (red) and CD11c (white) expression in microglia phagocytosing GFP-hTDP-43 (green) in the primary motor cortex of WT mice at $14 \mathrm{dpi}$; Scale bar, $10 \mu \mathrm{m}$. d, Representative images of microglia (Iba1, red) phagocytosis of GFP-hTDP-43 (green) in the primary motor cortex of WT but not TREM2 KO mice at $28 \mathrm{dpi}$, as indicated by the arrowheads; Scale 
bar, $20 \mu \mathrm{m}$. e, Quantification of hTDP-43 associated microglia (Iba1) in the primary motor cortex of WT and TREM2 KO mice at $28 \mathrm{dpi}$ ( $\mathrm{n}=7$ per group). Result indicates decreased hTDP-43 associated microglia in TREM2 KO mice. f, Analysis of co-localization of Iba1 (red curves) and GFP-hTDP-43 (green curves) in the primary motor cortex of WT and TREM2 KO mice at $28 \mathrm{dpi}$. Fluorescence intensity profiles of Iba1 and GFP-hTDP-43 show the distribution of fluorescence across the white dotted

arrows in (d). g, Representative images of co-localization of CD11c (white) with Iba1 (red) in microglia phagocytosing GFP-hTDP-43 (green) in the primary motor cortex of WT mice at $28 \mathrm{dpi}$; Scale bar, $10 \mu \mathrm{m}$. h, Analysis of co-localization of Iba1 (red curves), CD11c (grey curves) and GFP-hTDP-43 (green curves) in the primary motor cortex of WT mice at 28 dpi. Fluorescence intensity profiles of Iba1, CD11c, and GFP-hTDP-43 show the distribution of fluorescence across the yellow dotted arrows in (g). Data are represented as mean \pm SEM. Significances were calculated using Two-way ANOVA, Tukey's post-hoc analysis (b) and student t test (e); n.s., not significant; ${ }^{*} P<0.05,{ }^{* *} P<$ $0.01,{ }^{* * *} P<0.001$.

Fig 5. TREM2 deficiency leads to increased accumulation of pathological hTDP-43 protein.

a, Representative images of co-localization of p-TDP-43 (red) with GFP-hTDP-43 in the primary motor cortex of WT and TREM2 KO mice at $28 \mathrm{dpi}$, as indicated by white arrowheads; Scale bar, $100 \mu \mathrm{m}$. High magnification images as indicated by area in dotted white box showing on the right; Scale bar, $10 \mu \mathrm{m}$. b, Analysis of co-localization of p-TDP-43 (red curves) and GFP-hTDP-43 (green curves) in the primary motor cortex of WT and TREM2 KO mice at $28 \mathrm{dpi}$. Fluorescence intensity profiles of p-TDP-43 and GFP-hTDP-43 show the distribution of fluorescence across the yellow dotted arrow in (a). c-e, Quantification of hTDP-43 area (c, n=14 per group), p-TDP-43 area (d, n=10 per 
group) and GFP ${ }^{+}$particle size (e, n=10 per group) in the primary motor cortex of WT and TREM2 KO mice at $28 \mathrm{dpi}$. Results indicate higher hTDP-43, p-TDP-43 and GFP ${ }^{+}$in TREM2 KO mice. f, hTDP-43 particle size distribution in the primary motor cortex of WT and TREM2 KO mice at $28 \mathrm{dpi}$. Particles with size below $40 \mu \mathrm{m}^{2}$ or over $200 \mu \mathrm{m}^{2}$ are highlighted by blue ( $n=9$ per group). g, GFP-hTDP-43 and p-hTDP-43 (Ser403/404) immunoblots of the soluble fractions and Sarkosyl-insoluble fraction from primary motor cortex of WT and TREM2 KO mice at $28 \mathrm{dpi}$. Western blots were independently repeated four times ( $n=4$ per group). GAPDH was used as loading control. TREM2 KO mice show high level of GFP-hTDP-43 and p-hTDP-43 as compared to WT mice. Data represented as mean \pm SEM. Significance was calculated using student $t$ test; $n$.s., not significant; ${ }^{*} P$ $<0.05,{ }^{* *} P<0.01$.

Fig 6. hTDP-43 interacts with TREM2 in vitro and in vivo in mouse brain.

a, hTDP-43 was co-immunoprecipitated with myc-hTREM2 in HEK 293 cells overexpressing hTREM2. Experiments were independently repeated three times. b, Annotated MS/MS spectra of $V^{124}$ LVEVLADPLDHR $^{136}$ from hTREM2 and $T^{103}$ SDLIVLGLPWK ${ }^{114}$ from hTDP-43 identified in anti-hTREM2 immunoprecipitates from HEK 293 cells. c, Representative images of hTDP-43 expression in cytosol in the primary motor cortex of rNLS mice at 3 weeks off DOX diet; Scale bar, $20 \mu \mathrm{m}$. d, hTDP-43 immunoblots of primary motor cortex of rNLS mice at 3 weeks off DOX diet. e, rNLS mice showed clasping phenotype at 2 weeks off DOX diet. f, Hindlimb clasping response scores over 4 weeks off DOX diet followed by 2 weeks DOX on ( $n=12$ per group). g, Representative images of microglia (Iba1, red) in the primary motor cortex of indicated groups at 4 weeks off DOX diet followed with 2 weeks DOX on. $\mathbf{h}$, Quantification of microglial density ( $n=10$ per group) in the primary motor cortex of indicated groups at 4 weeks off DOX diet followed by 2 weeks DOX on. Results indicate 
increased microglia number in rNLS mice. i, Endogenous mTREM2 was co-immunoprecipitated with hTDP-43 in the motor cortex of rNLS mice using mTREM2 antibody at 4 weeks off DOX diet followed with 2 weeks DOX on. Experiments were independently repeated three times. j, Annotated MS/MS spectra of $\mathrm{N}^{99}$ LQAGDAGLYQcQSLR $^{114}$ (c is carbaminomethylated cysteine) from mouse TREM2 and $\mathrm{F}^{152}$ TEYETQVK ${ }^{160}$ from hTDP-43 in anti-mTREM2 immunoprecipitates from rNLS mouse cortex tissue at 4 weeks off DOX diet followed by 2 weeks DOX on. Data represented as mean \pm SEM. Significances were calculated using Two-way ANOVA, Tukey's post-hoc analysis (f) and student $\mathrm{t}$ test (h); n.s., not significant; ${ }^{*} P<0.05,{ }^{* *} P<$ $0.01,{ }^{* * *} P<0.001$.

Fig 7. hTDP-43 interacts with TREM2 in human tissues and in silico.

a, TREM2 immunoblots of frozen autopsied spinal cord specimens of ALS patients and age matched controls. b, Quantification of TREM2 level relative to GAPDH ( $n=12$ for control, $n=16$ for ALS patients). Results indicate increased expression of TREM2 in ALS patient samples as compared to non-neurological controls. c, Endogenous TDP-43 of frozen autopsied spinal cord specimens from ALS patients was co-immunoprecipitated with TREM2. Experiments were independently repeated three times. d-g, Cartoon models of the human TREM2 extracellular ligand-binding domain in complex with four low-complexity domain fragments of TDP-43. $\mathbf{h}$, Surface model of the TREM2 complex showing overlapping binding sites of GFN and NFG and distinct binding sites of NFG, AMM, and SWG. (NFG: $N^{312} F_{G A F S}{ }^{317} ; \quad$ AMM: $A^{321} M_{M A A A}{ }^{326}$; SWG: $\mathrm{S}^{333}$ WGMMGMLASQ $^{343}$; GFN: $\mathrm{G}^{396} \mathrm{FNGGFG}^{402}$ ). Data represented as mean $\pm \mathrm{SEM}$. Significances were calculated using student $\mathrm{t}$ test; $n$.s., not significant; ${ }^{*} P<0.05$, ${ }^{* *} P<$ $0.01,{ }^{* * *} P<0.001$. 


\section{Extended Data Figure Legends}

\section{Extended Data Fig 1. Characterizations of hTDP-43 expression in a neonatal TDP-43 mouse model.}

GFP-hTDP-43 expression was induced via intracerebroventricular injection of AAV9.CAG.hTDP-43-GFP in neonatal mice (AAV9.CAG.GFP as control). a, Representative image of brain GFP-hTDP-43 expression in C57/BL6 (WT) mice at 21 days post-infection (dpi). Primary motor cortex (M1), supplementary motor area (M2) and lateral ventricle (LV) are separated by dashed line. Inset shows seeding hTDP-43 expression at higher magnification as indicated by the area in dotted yellow box; Scale bar, $100 \mu \mathrm{m}$. b, Representative image of spinal cord GFP-hTDP-43 expression in C57/BL6 (WT) mice at 21 days post-infection (dpi); Scale bar, $200 \mu \mathrm{m}$. c, Representative image of brain GFP-hTDP-43 expression in the brain regions of cerebral cortex, hippocampus, thalamus and brainstem in WT mice at 21 days post-infection (dpi); Scale bar, $100 \mu \mathrm{m}$. d, Quantification of GFP-hTDP-43 expression in the brain regions of cerebral cortex, hippocampus, thalamus, brainstem and spinal cord (SC) in WT mice at 21 days post-infection (dpi). e, Representative images of co-immunostaining of GFP-hTDP-43 (green) with NeuN (red), Iba1 (red), GFAP (red) or CNPase (red) in the primary motor cortex of WT mice expressing hTDP-43 at 21 dpi. f, Quantification of the percentage of co-localization of GFP-hTDP-43 with NeuN, Iba1, GFAP or CNPase in the primary motor cortex of WT mice expressing hTDP-43 at 21 dpi ( $n=7$ per group). $\mathbf{g}$, Representative images of $\mathrm{p}$-hTDP-43 expression in the brain regions of hippocampus, thalamus, brainstem at $35 \mathrm{dpi}$; Scale bar, $20 \mu \mathrm{m}$. Data represented as mean \pm SEM. 
Extended Data Fig 2. Characterizations of motor deficits and neuronal loss in a neonatal TDP-43 mouse model.

GFP-hTDP-43 expression was induced via intracerebroventricular injection of AAV9.CAG.hTDP-43-GFP in neonatal mice (AAV9.CAG.GFP as control). a, Body weights were measured separately for male and female in indicated groups at $35 \mathrm{dpi}(\mathrm{n}=$ 13 per group). b, Representative traces of locomotor activity in an open field test at 35 dpi. c, Average total distance traveled during open field test ( $n=25$ per group). d, Representative immunostaining images of NeuN (red) with DAPI (blue) in the primary motor cortex of indicated groups at $35 \mathrm{dpi}$. M1 and M2 are separated by dashed line. Insets show NeuN staining in layer 5 of primary motor cortex at higher magnification as indicated by the area in dotted white box; Scale bar, $20 \mu \mathrm{m}$. e, Quantification of NeuN positive cells in the primary motor cortex of indicated groups at $35 \mathrm{dpi}$ ( $\mathrm{n}=10$ per group).

Data represented as mean \pm SEM. Significance was calculated using Two-way ANOVA, Tukey's post-hoc analysis; $n$.s., not significant; ${ }^{* * *} P<0.001$.

Extended Data Fig 3. TREM2 deficiency leads to increased accumulation of hTDP-43 protein.

hTDP-43 protein expression was induced via intracerebroventricular injection of AAV9.CAG.hTDP-43 in neonatal mouse (AAV9.CAG.Empty as control). a, Schematic representation of the velocity sedimentation protocol for separating soluble from Sarkosyl-insoluble aggregated proteins from an aqueous homogenate of brain tissue. $\mathbf{b}$, Whole brain hTDP-43 and p-hTDP-43 (Ser403/404) immunoblots of the soluble fraction of WT and TREM2 KO mice at 70 dpi. c, Whole brain p-hTDP-43 immunoblots of the Sarkosyl-insoluble fraction of WT and TREM2 KO mice at 70 dpi. Western blots were independently repeated twice with $n=4$ for each group. 
Extended Data Fig 4. TREM2 deficiency attenuates hTDP-43-induced microglial activation.

hTDP-43 protein expression was induced via intracerebroventricular injection of AAV9.CAG.hTDP-43 in neonatal mouse (AAV9.CAG.Empty as control). a, Representative images of GFP-expressing microglia in the primary motor cortex of indicated groups at $35 \mathrm{dpi}$. M1 and M2 are separated by dashed line. Insets show microglia at higher magnification as indicated by the area in white box; Scale bar, $50 \mu \mathrm{m}$. b, c, Quantification of GFP-expressing microglia number (b) and soma size (c) in the primary motor cortex of indicated groups at $35 \mathrm{dpi}(\mathrm{n}=10$ per group). hTDP-43 increased microglia number in both $\mathrm{CX} 3 \mathrm{CR} 1^{\mathrm{GFP} /+}: \mathrm{WT}$ and $\mathrm{CX} 3 \mathrm{CR} 1^{\mathrm{GFP} /+}: \mathrm{TREM} 2 \mathrm{KO}$ mice. However, increased soma size was observed only in hTDP-43 CX3CR $1^{\mathrm{GFP} /+}$ :WT mice. d, Representative images of co-localization of CD11c (white) with Iba1 (red) in microglia phagocytosing GFP-hTDP-43 (green) in the primary motor cortex of WT group at $35 \mathrm{dpi}$. White arrowheads indicate phagocytic puncta of GFP-hTDP-43; Scale bar, 20 $\mu \mathrm{m}$. e, Pie chart representing the percentage of microglia phagocytosing GFP-hTDP-43 (green, phagocytic microglia) and those non-phagocytic microglia in the primary motor cortex of WT group at $35 \mathrm{dpi}$. Data represented as mean \pm SEM. Significance was calculated using two-way ANOVA followed by Tukey's post hoc test; $n$.s., not significant; ${ }^{* * *} P<0.001$.

Extended Data Fig 5. Characterizations of hTDP-43 expression mouse model via local virus injection in the primary motor cortex of adult mice.

GFP-hTDP-43 or hTDP-43 was expressed in the primary motor cortex of 2-month-old mice via stereotactic intracerebral injection of AAV9.CAG.hTDP-43-GFP or AAV9.CAG.hTDP (AAV9.CAG.GFP or AAV9.CAG.Empty as control). a, Schematic 
picture showing stereotactic virus injection site (upper). Representative image of GFP expression in the primary motor cortex of 2-month-old WT mice at $14 \mathrm{dpi}$ (lower); Dashed lines indicate the borders of layer 4\&5; Scale bar, $100 \mu \mathrm{m}$. b, Representative images of translocation of GFP-hTDP-43 from nuclear to cytosol (white arrowhead) and dendrites (white arrow) in motor neurons of 2-month-old WT mice expressing GFP-hTDP-43 via stereotactic intracerebral injection at $14 \mathrm{dpi}$. AAV1.CAG.tdTomato virus was co-injected to visualize neurons; Scale bar, $10 \mu \mathrm{m}$. c, Representative images of GFP-hTDP-43 expression in the primary motor cortex at $14 \mathrm{dpi}$; Scale bar, $100 \mu \mathrm{m}$. d, Quantification of GFP-hTDP-43 density in the primary motor cortex of WT and TREM2 mice at $14 \mathrm{dpi}(\mathrm{n}=7$ per group). e, GFP-hTDP-43 immunoblots of primary motor cortex of WT and TREM KO mice at $14 \mathrm{dpi}$. Western blots were independently repeated four times ( $\mathrm{n}=8$ per group). GAPDH was used as loading control. f, Representative images of GFP-expressing microglia in the primary motor cortex of indicated groups at $14 \mathrm{dpi}$; Scale bar, $100 \mu \mathrm{m} . \mathbf{g}$, Quantification of GFP-expressing microglia number in the primary motor cortex of indicated groups at $14 \mathrm{dpi}(\mathrm{n}=12$ per group). $\mathrm{h}$, Representative images of microglia (Iba1, red) phagocytosing GFP-hTDP-43 (green) in the primary motor cortex of WT but not TREM2 KO mice at $28 \mathrm{dpi}$; Scale bar, $100 \mu \mathrm{m}$. Data represented as mean \pm SEM. Significances were calculated using student t test (d) and Two-way ANOVA, Tukey's post-hoc analysis (g); n.s., not significant; ${ }^{*} P<0.05,{ }^{* *} P<0.01,{ }^{* * *} P<0.001$.

\section{Extended Data Fig 6. TREM2 deficiency facilitates hTDP-43-induced} neurodegeneration.

GFP-hTDP-43 or hTDP-43 was expressed in the primary motor cortex of 2-month-old mice via stereotactic intracerebral injection of AAV9.CAG.hTDP-43-GFP or AAV9.CAG.hTDP (AAV9.CAG.GFP or AAV9.CAG.Empty as control). a, Representative images of NeuN immunostaining (red) in the primary motor cortex of indicated groups at 
$28 \mathrm{dpi}$. Scale bar, $100 \mu \mathrm{m}$. b, Quantification of $\mathrm{NeuN}^{+}$cells in the primary motor cortex of indicated groups at $28 \mathrm{dpi}$ ( $\mathrm{n}=5$ per group). hTDP-43 induced neurodegeneration was more prominent in TREM2 KO mice as compared with WT mice. c, Representative images of microglia (GFP) interaction with $\mathrm{NeuN}^{+}$neurons (red) in the primary motor cortex of indicated groups at $28 \mathrm{dpi}$. Scale bar, $50 \mu \mathrm{m}$. d, Quantification of microglia-neuron interaction in the primary motor cortex of indicated groups at $28 \mathrm{dpi}(\mathrm{n}=$ 4 per group). Results show that hTDP-43 expression increased microglia and neuronal interaction in WT mice unlike in TREM2 KO mice. e, Representative images of CD11C (white) expression in microglia (CX3CR1-GFP, green) interacting with $\mathrm{NeuN}^{+}$neurons (red) in the primary motor cortex of WT groups at $28 \mathrm{dpi}$. Scale bar, $20 \mu \mathrm{m}$. Data represented as mean \pm SEM. Significance was calculated using two-way ANOVA followed by Tukey's post hoc test; $n$.s., not significant; ${ }^{* *} P<0.01,{ }^{* *} P<0.001$.

\section{Extended Data Fig 7. TDP-43 can be released from neurons and interact with TREM2 in vitro.}

a, Representative images of human iPSC derived neurons infected with AAV9.CAG.hTDP-43-GFP virus or control virus at 21dpi. AAV1.CAG.tdTomato virus used to visualize neurons; Scale bar, $100 \mu \mathrm{m}$. Inserts show neuron morphology at high magnification as indicated by the area in dotted white box. White arrowheads indicate GFP-hTDP-43 translocation in neurites. b, Immunoblots of TDP-43 contained within collected culture media of iPSCs (left) or in pulled down fractions by bead-immobilized GFP antibody (right). c, Representative images of HEK 293 cells co-transfected with myc-tagged human TREM2 (myc-hTREM2) and GFP-hTDP-43 C terminal fragment (residues 216-414) or GFP control plasmids. Scale bar, $20 \mu \mathrm{m}$. d, myc-hTREM2 was co-immunoprecipitated with GFP-hTDP-43 in HEK 293 cells using bead-immobilized GFP antibody. Experiments were independently repeated three times. e, GFP-hTDP-43 
was co-immunoprecipitated with myc-hTREM2 in HEK 293 cells using bead-immobilized myc antibody. Experiments were independently repeated three times.

\section{Extended Data Fig 8. Full-length Western blot images.}

a, Full-length blot image for Figure $5 \mathrm{~g}$. b, Full-length blot image for Figure 6a. c, Full-length blot image for Figure 6f. d, Full-length blot image for Figure $6 \mathrm{i}$.

\section{Extended Data Fig 9. Full-length Western blot images.}

a, Full-length blot image for Figure 7a. b, Full-length blot image for Figure 7c. c, Full-length blot image for Extended Data Figure 3b. d, Full-length blot image for Extended Data Figure 3c. e, Full-length blot image for Extended Data Figure 5d.

\section{Extended Data Fig 10. Full-length Western blot images.}

a, Full-length blot image for Extended Data Figure 7d. b, Full-length blot image for Extended Data Figure 7e. 
a

Survival rate

Hindlimb clasping score
Neuronal loss

TDP-43 pathology

Microglia response b

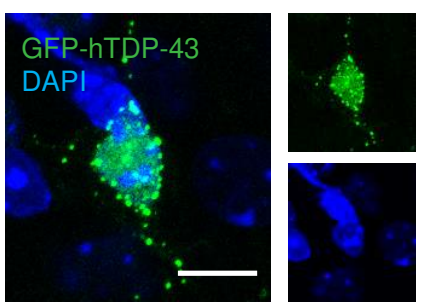

ICV injection on PO

5 weeks

Rotarod test

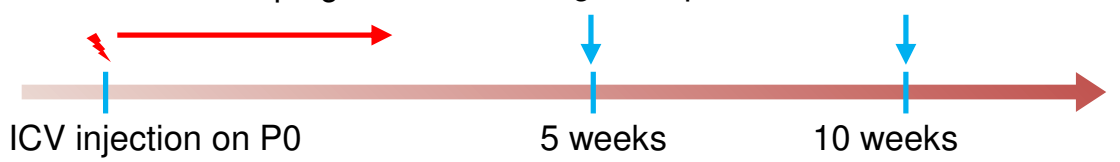

C

Motor cortex

AAV-Control
hTDP-43

\section{AAV-hTDP-43}

d
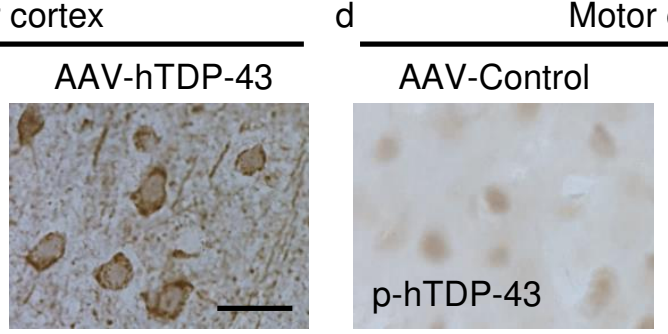

Motor cortex
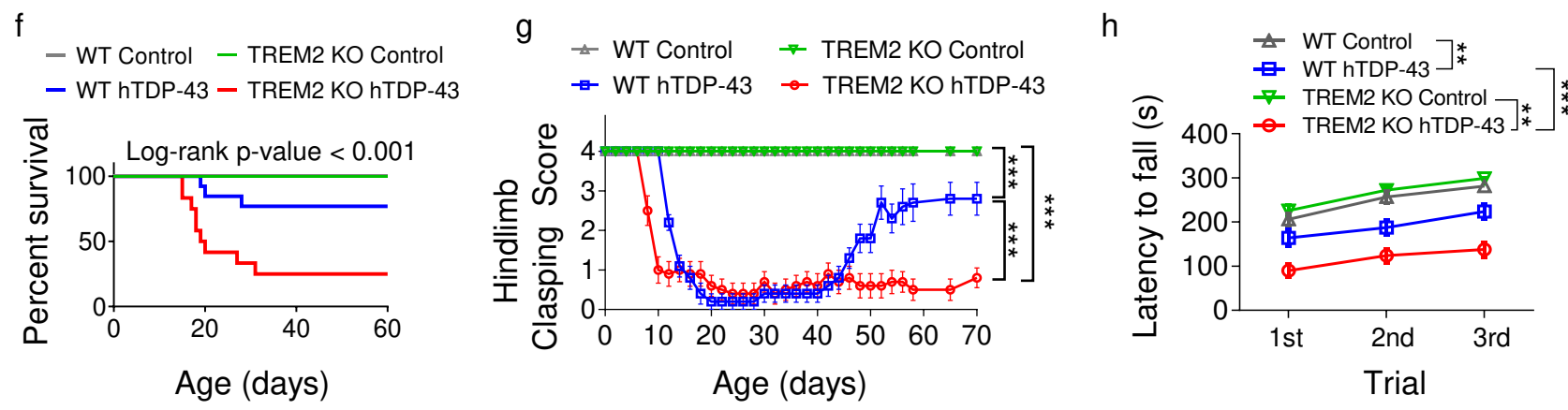

\section{,}

WT
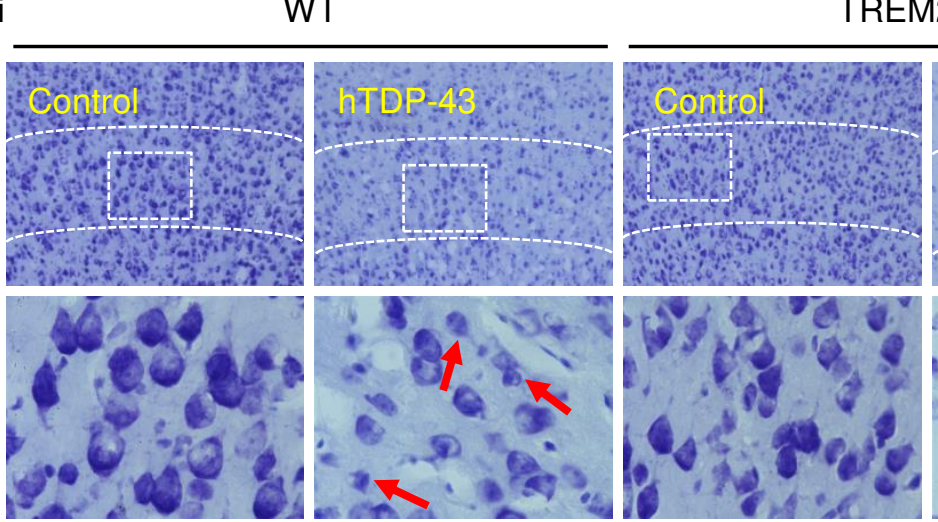

TREM2 KO

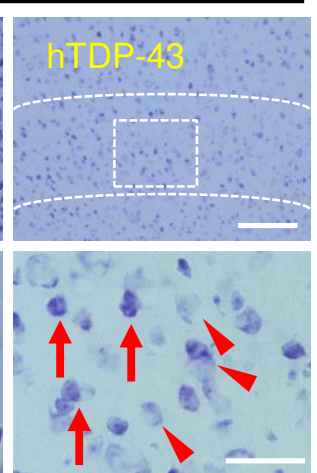

- Control

$\triangle$ hTDP-43

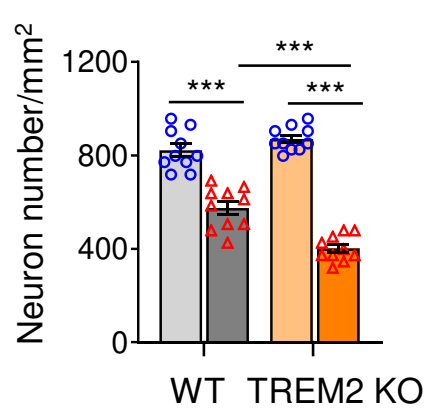


a

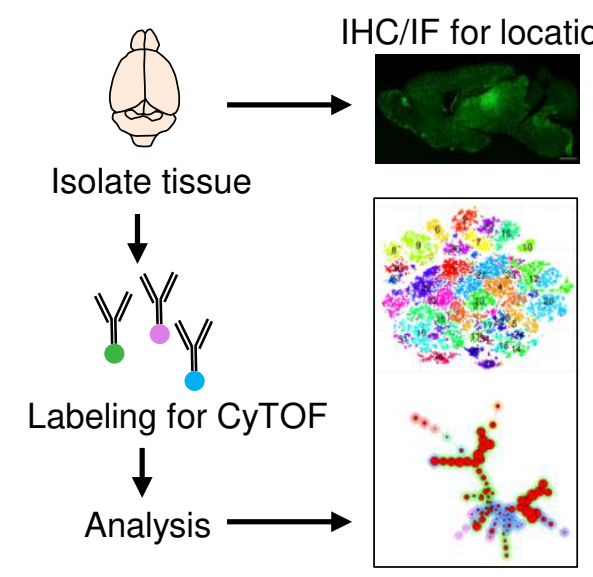

C

\section{CD45+CD11 ${ }^{+}$}

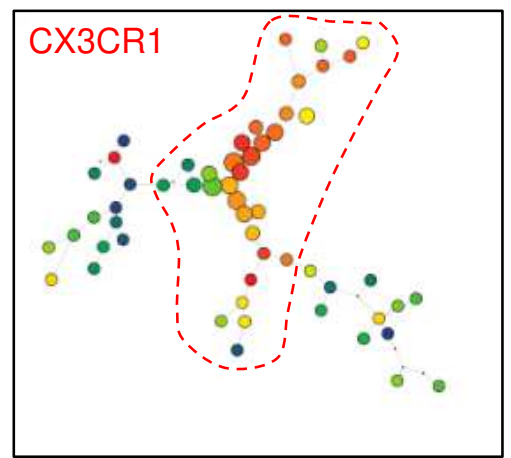

e

$$
\begin{array}{ll}
\text { - } & \text { Control } \\
\text { 4 } & \text { hTDP-43 }
\end{array}
$$

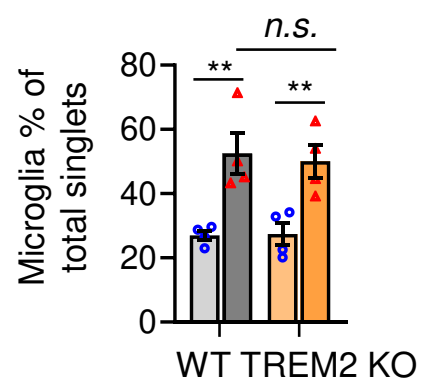

g

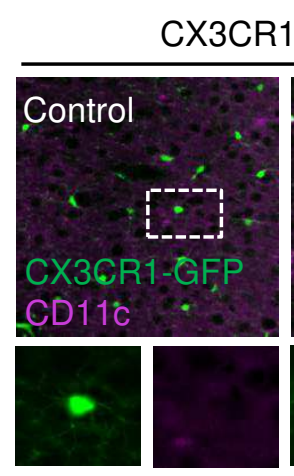

b

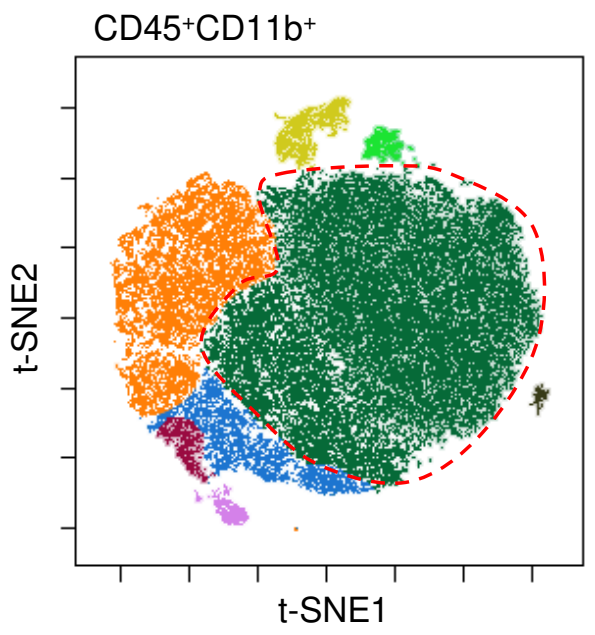

d

Microglia
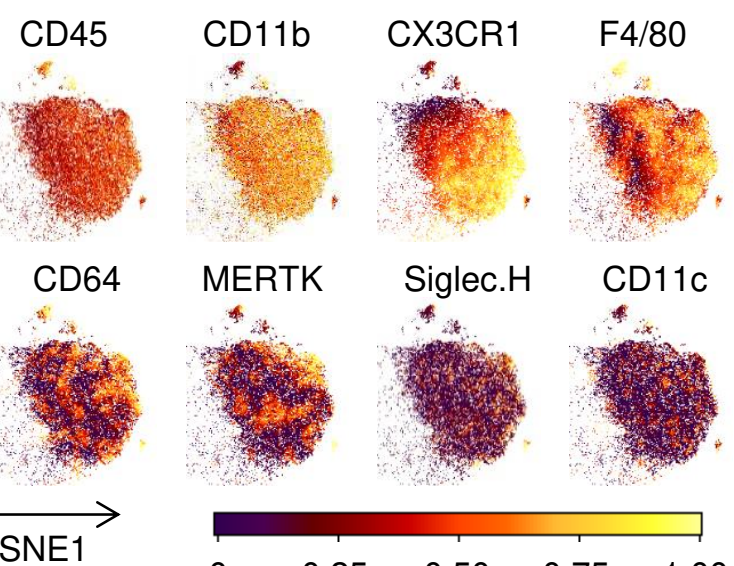

High

Low
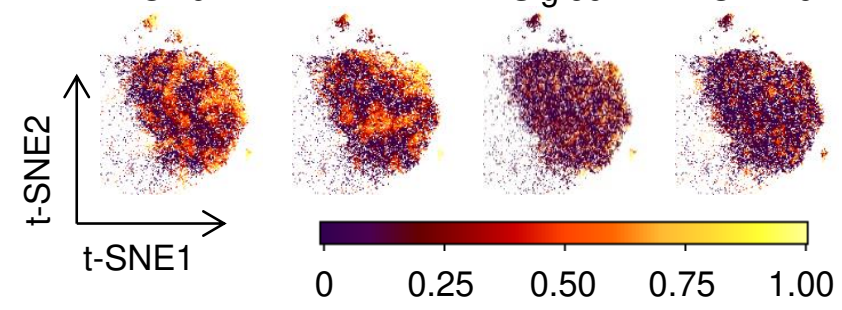
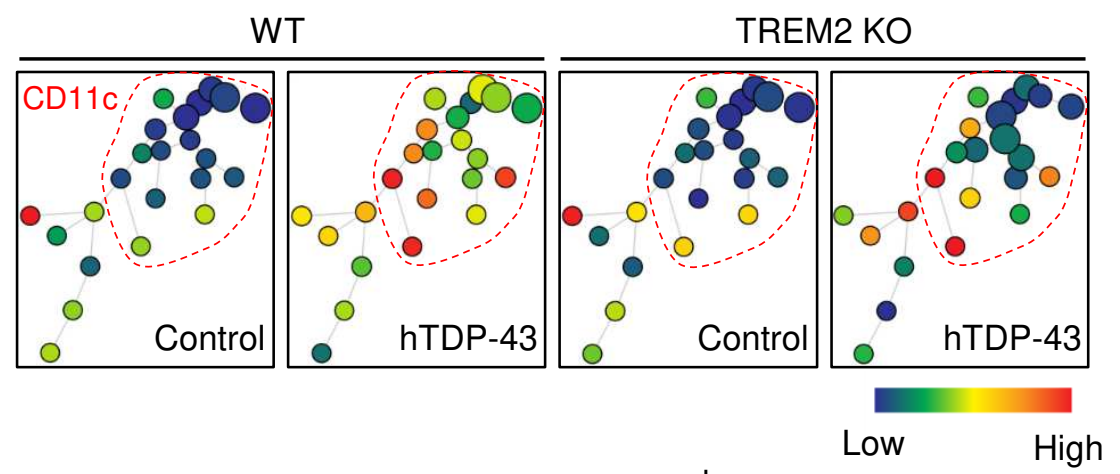

CX3CR1 $1^{\mathrm{GFP} /+}:$ TREM2 KO

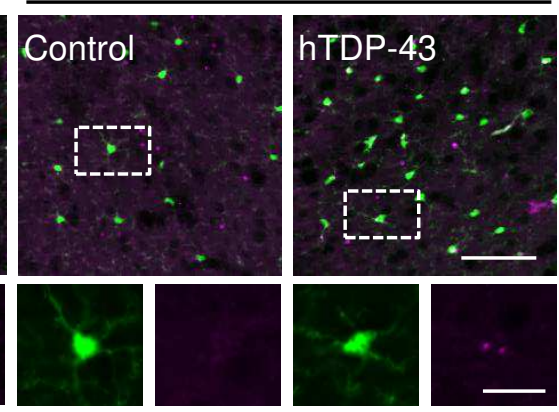

h

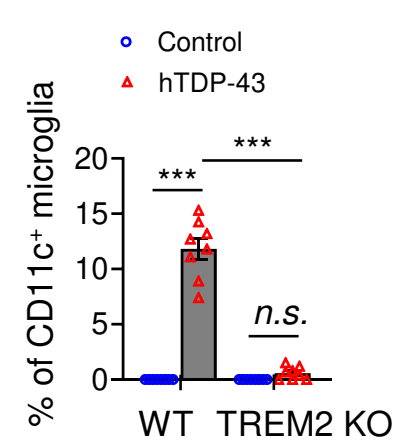


8 weeks old

$$
\begin{aligned}
& \text { Intracerebral } \\
& \text { virus injection }
\end{aligned}
$$

Microglia function

TDP-43 pathology

Neuronal loss

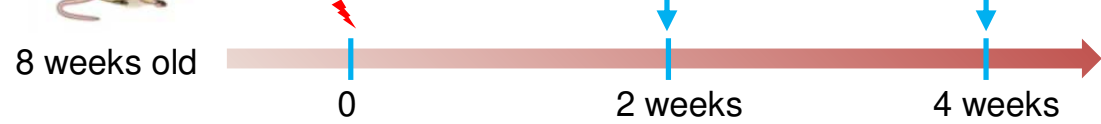

b
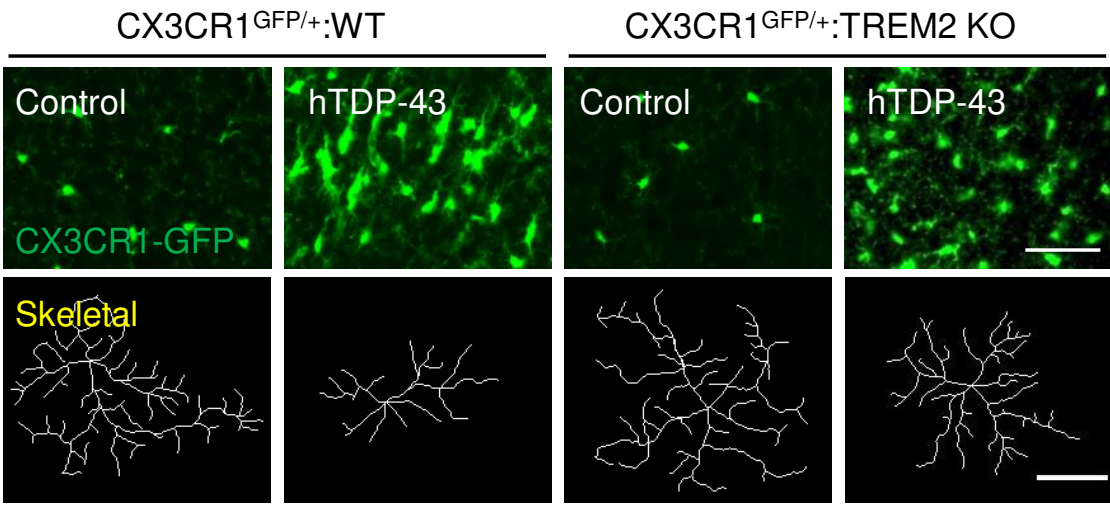

f

CX3CR1 $1 \mathrm{GFP} /+:$ CX3CR1GFP/+:

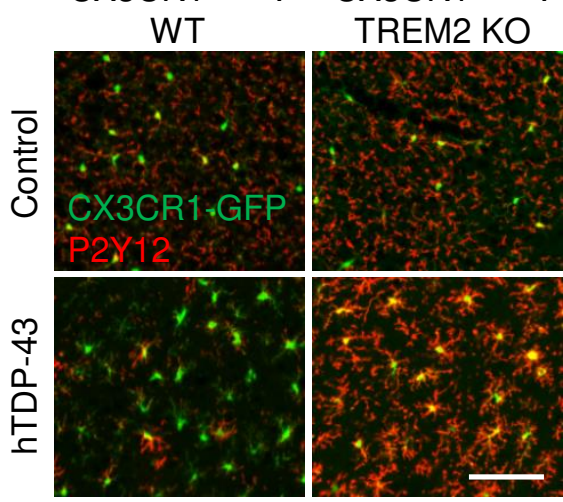

9 CX3CR1GFP/+.
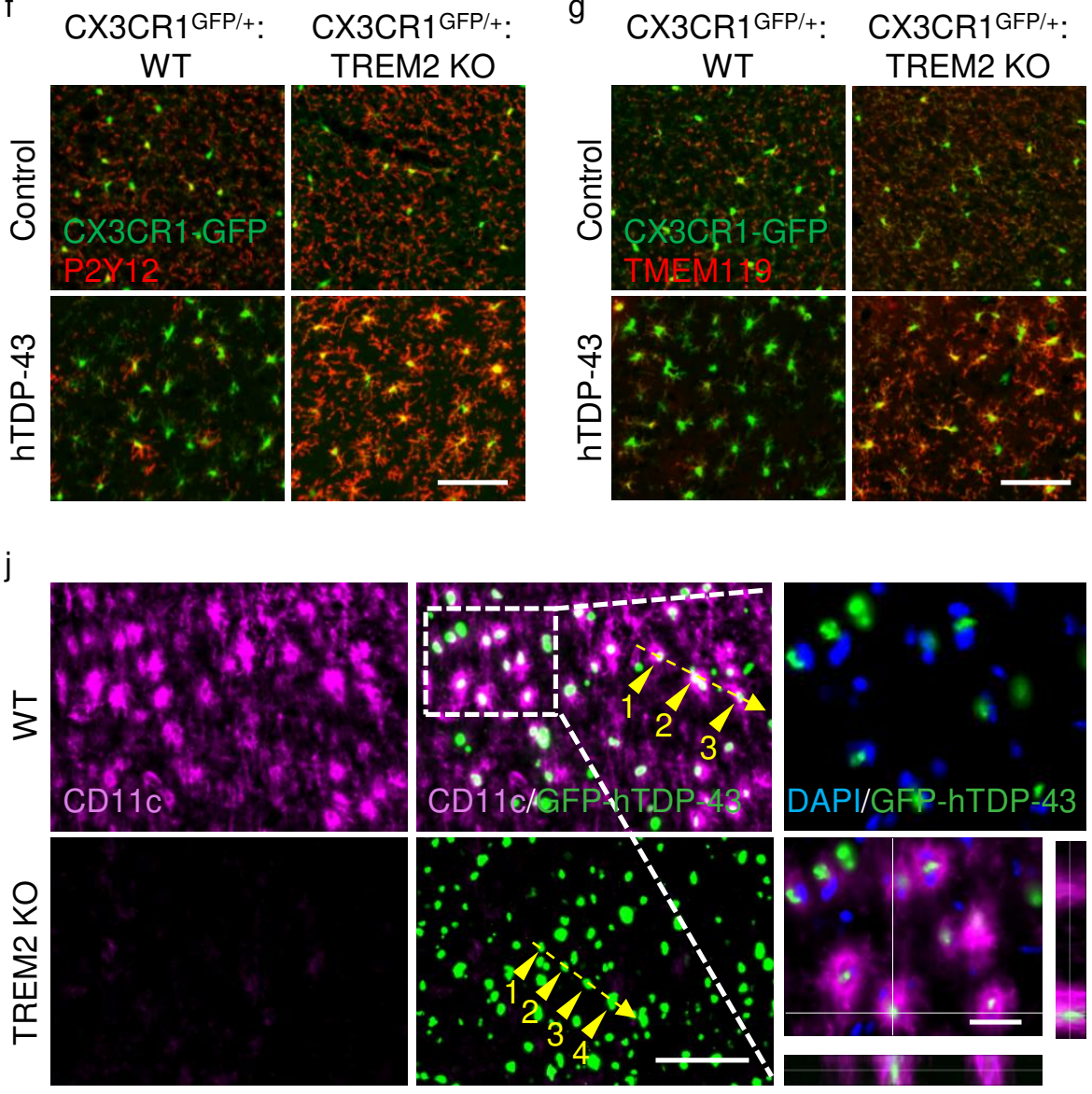

k
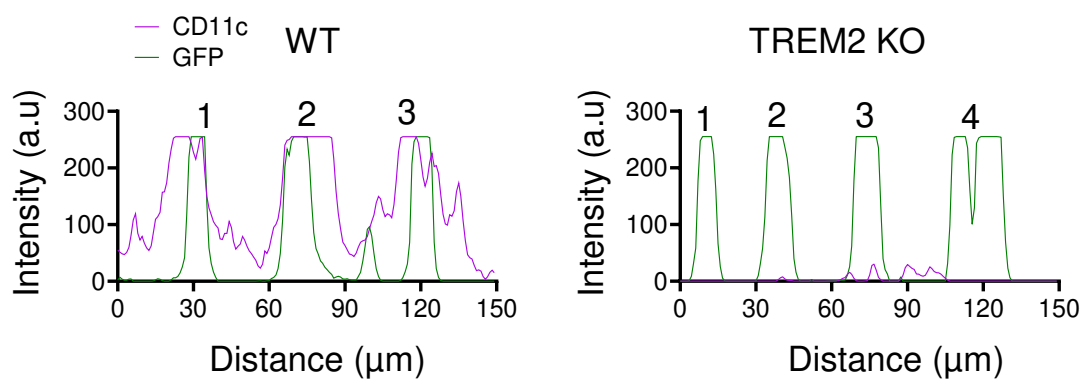

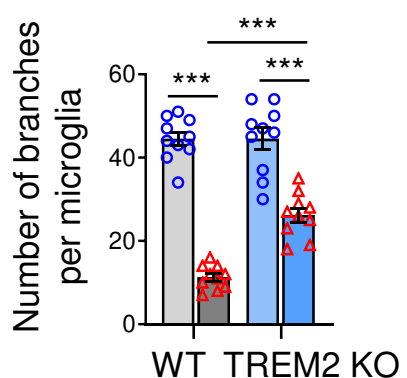

d

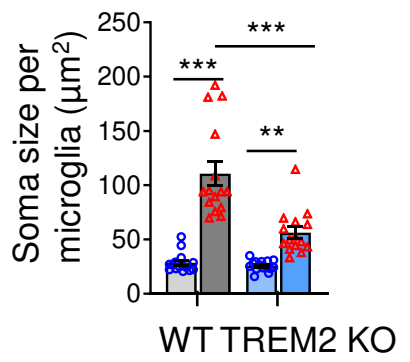

e
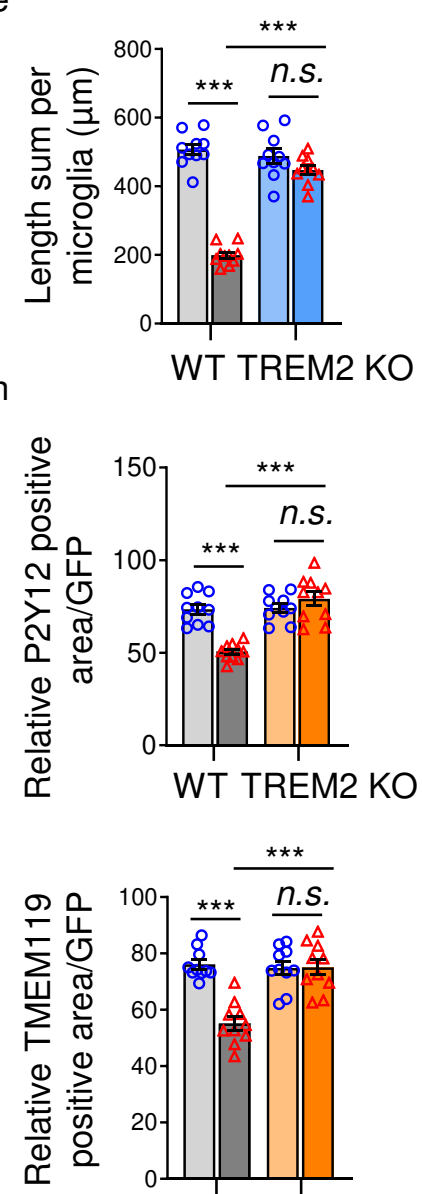

WT TREM2 KO

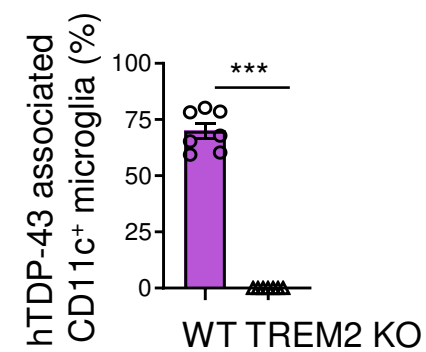


a
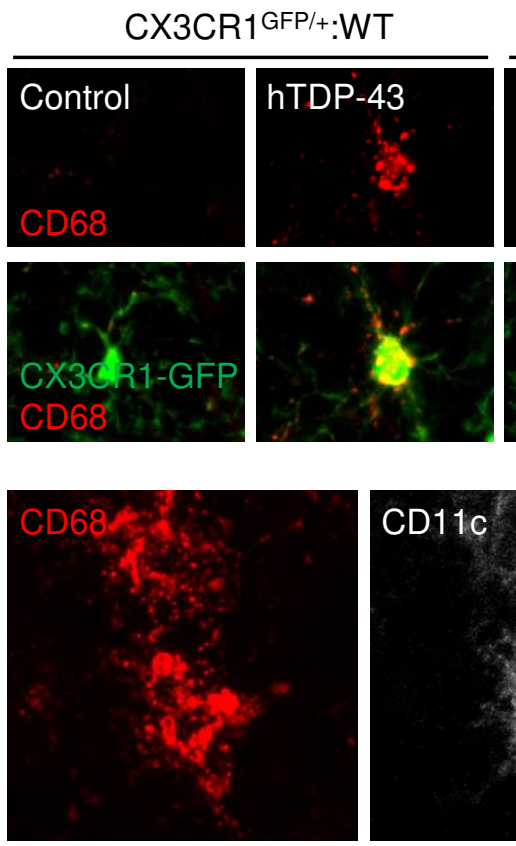

d

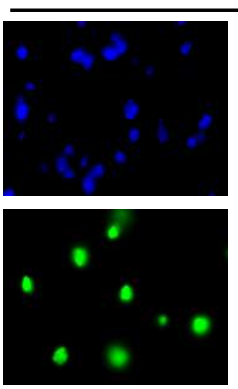

WT

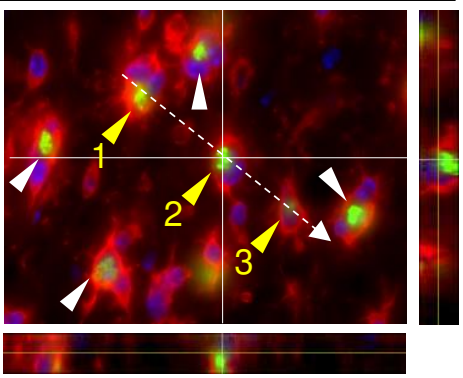

CX3CR1 $1^{\mathrm{GFP} /+}:$ TREM2 KO

\begin{tabular}{c}
\hline Control \\
hTDP-43 \\
\end{tabular}
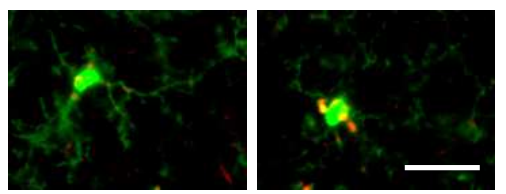

b

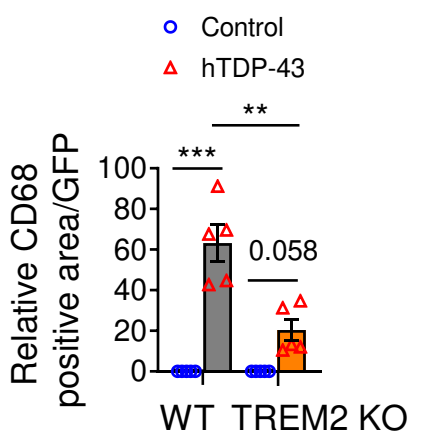

DAPI/lba1/GFP-hTDP-43
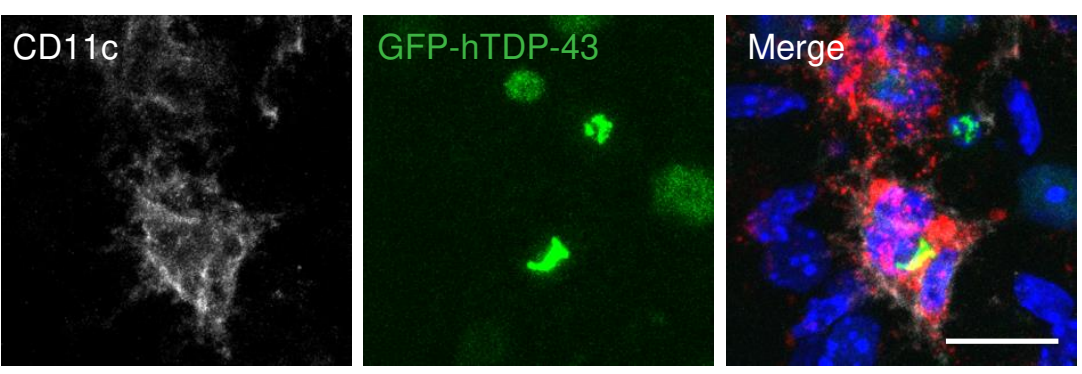

TREM2 KO
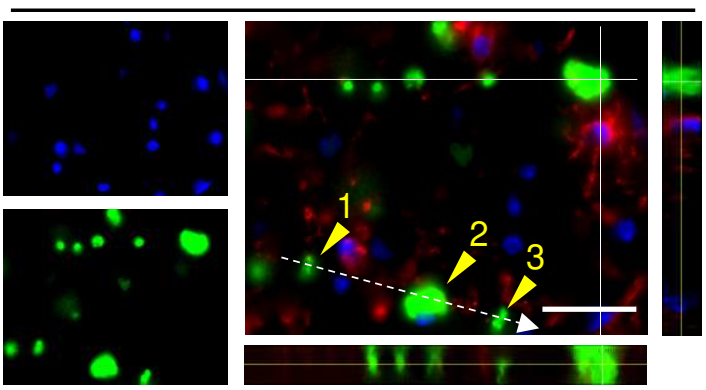

e

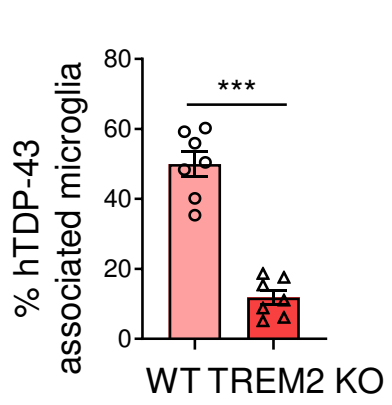

g

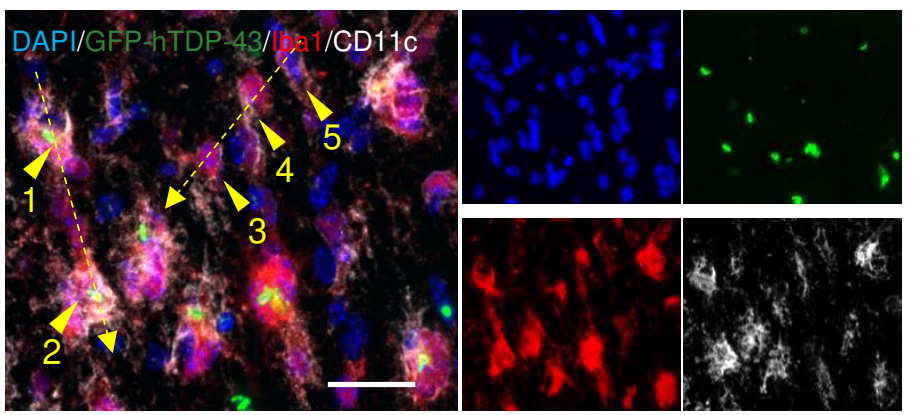

- Iba1 WT

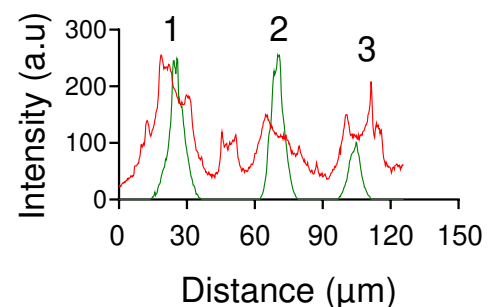

Distance $(\mu \mathrm{m})$
TREM2 KO
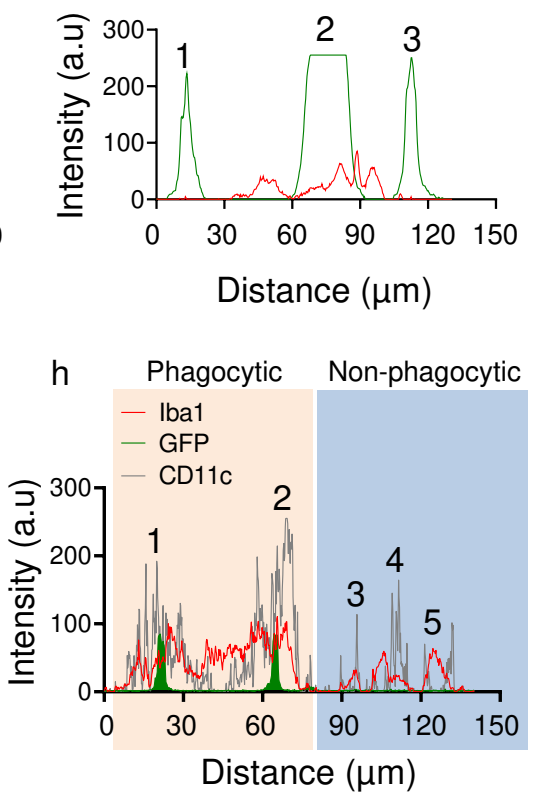
a
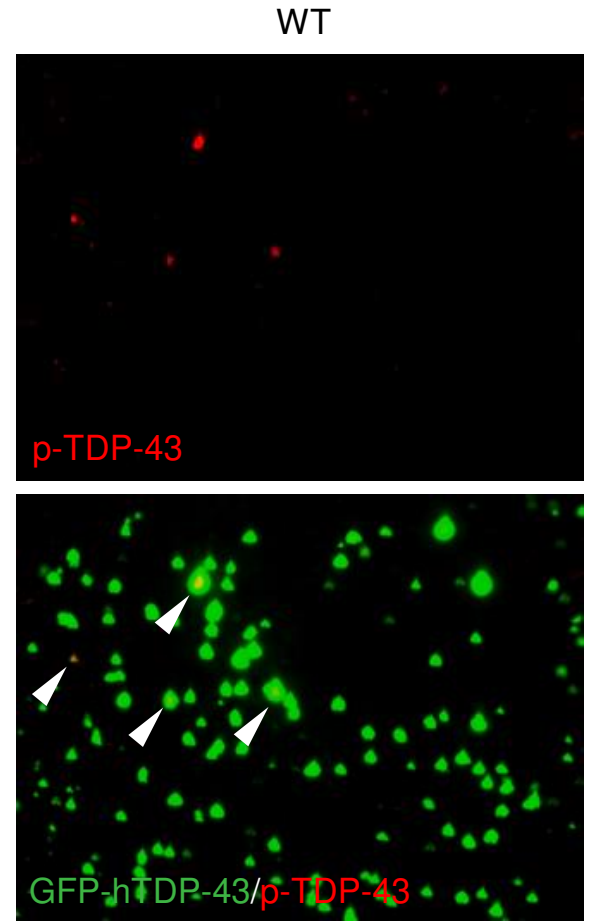

b

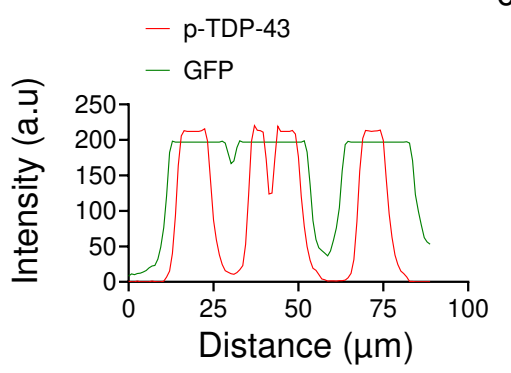

$c$

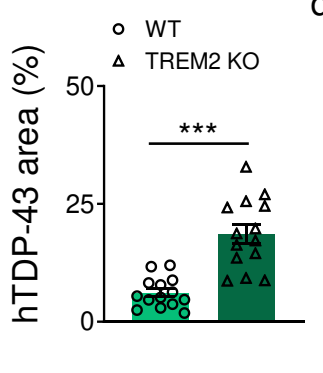

TREM2 KO
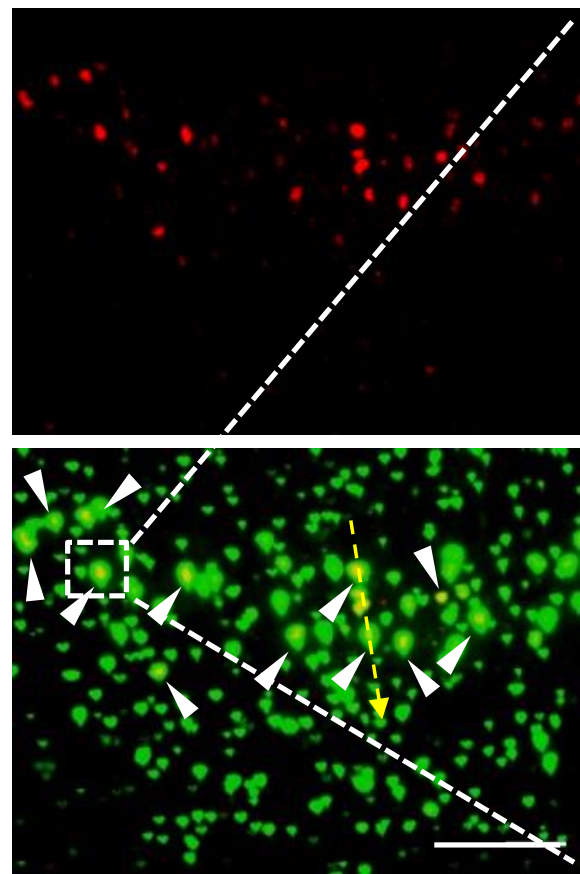

d

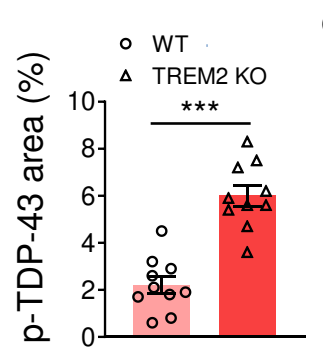

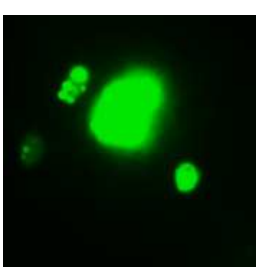
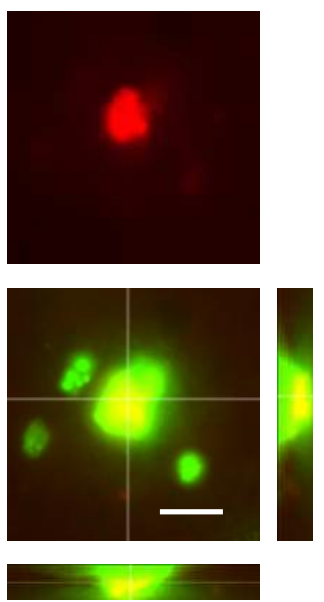

e

- WT $\Delta$ TREM2 KO

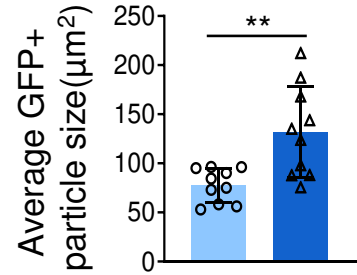

f

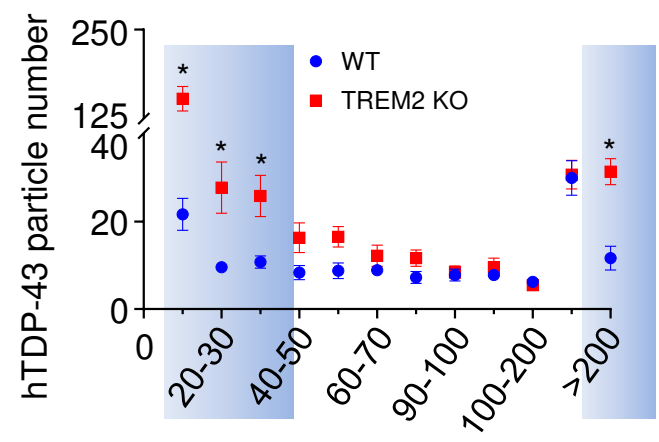

Particle size $\left(\mu \mathrm{m}^{2}\right)$ g

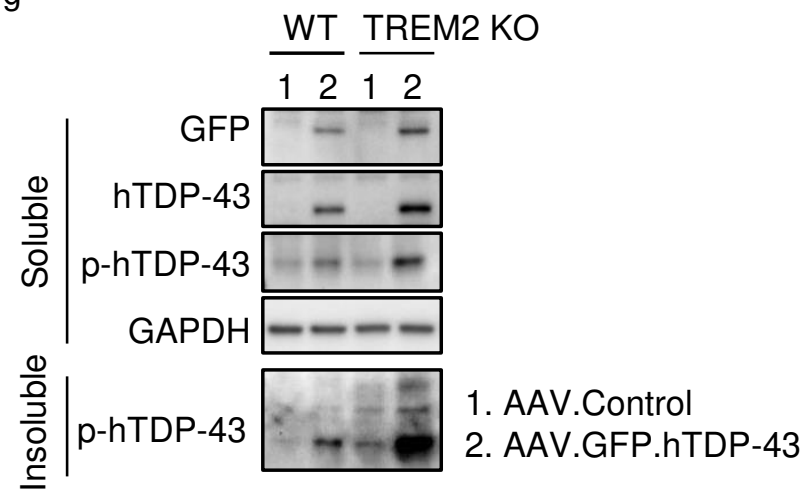


a

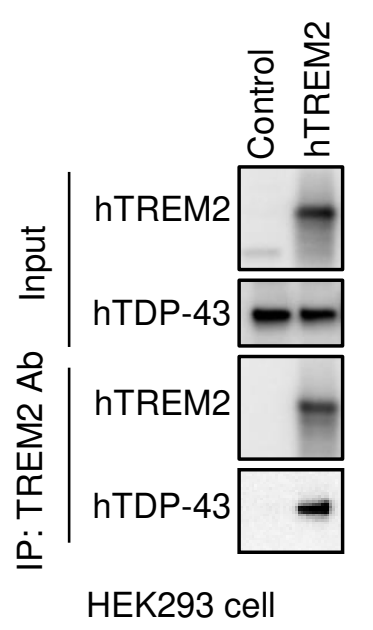

C

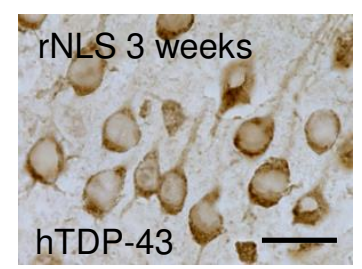

d Control rNLS

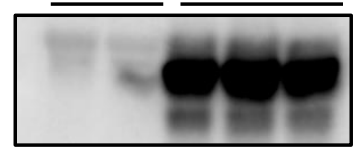

hTDP-43

g
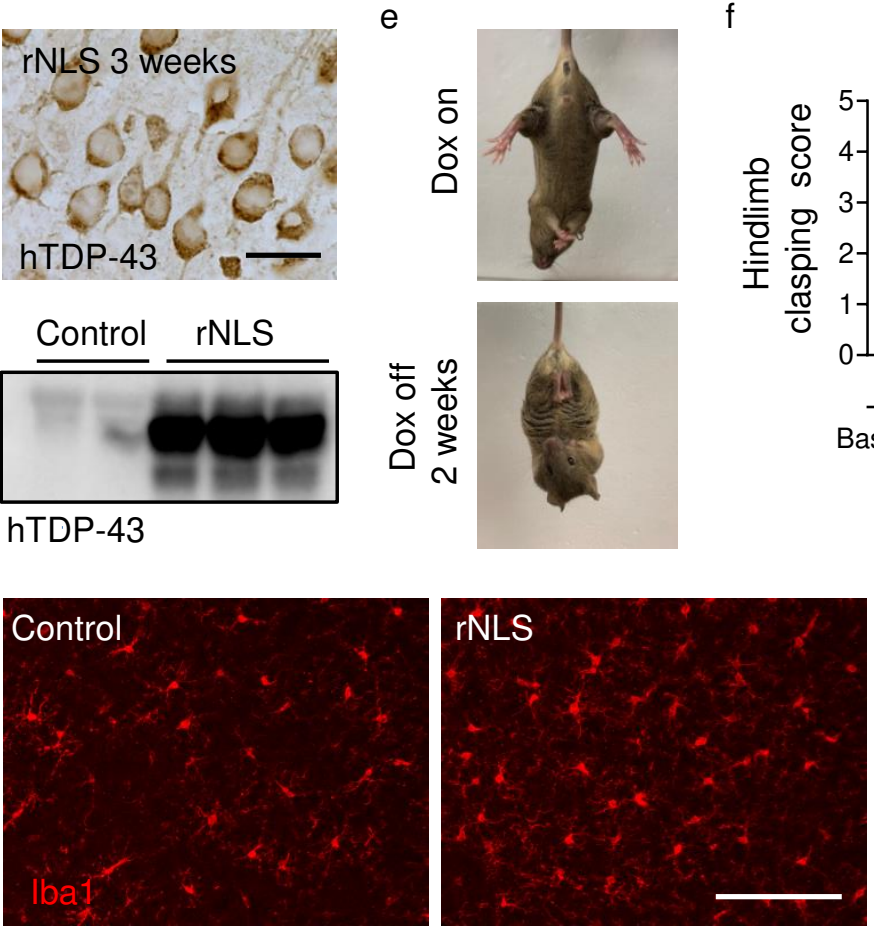

4 weeks DOX off +2 weeks DOX on

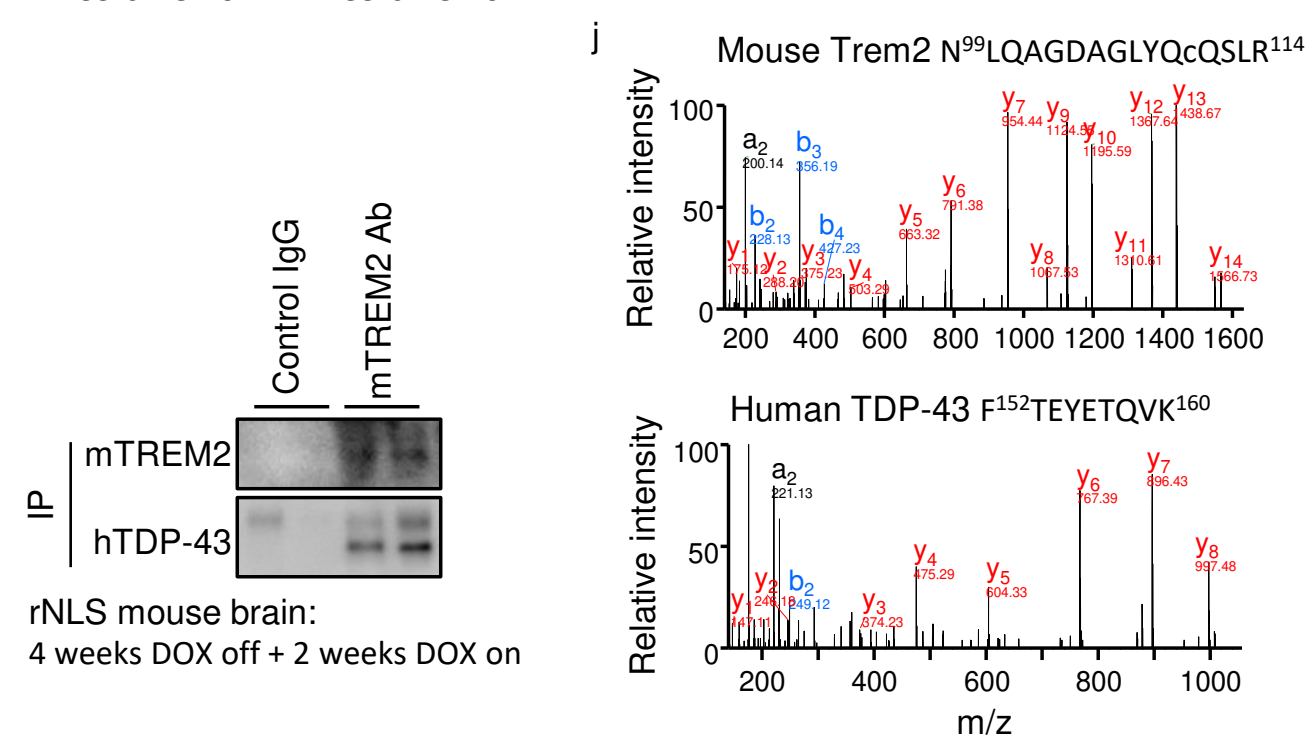

rNLS mouse brain:

4 weeks DOX off +2 weeks DOX on

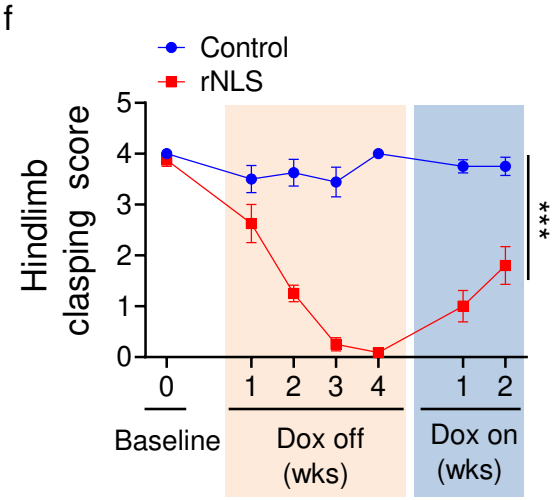

Human TREM2 V'124LVEVLADPLDHR ${ }^{136}$

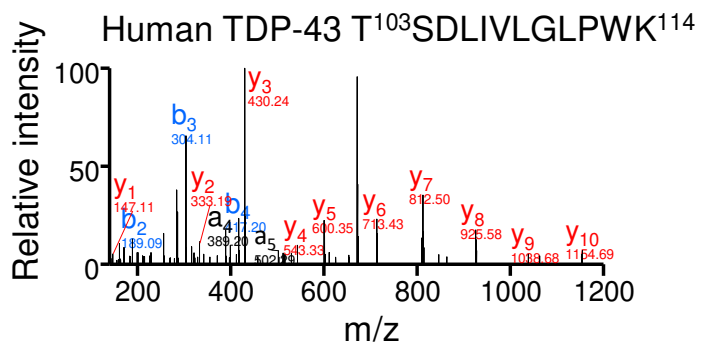

$\mathrm{h}$

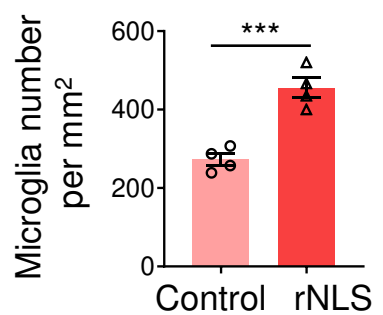


a

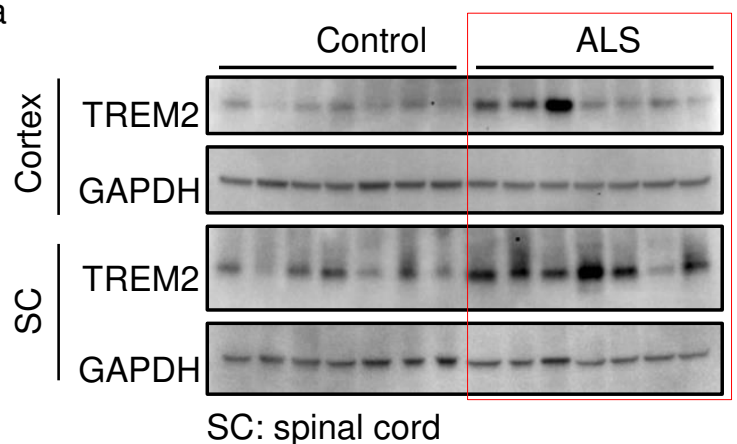

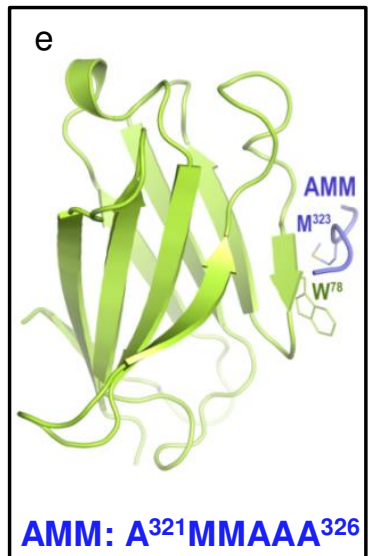

b

C

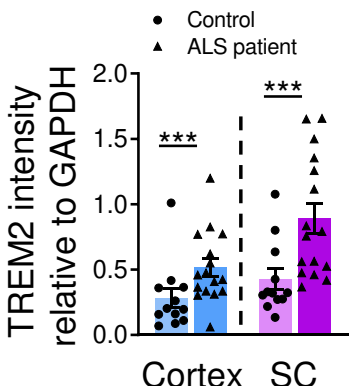

Control ALS

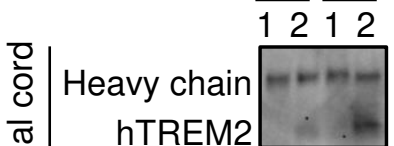

Heavy chain

$\ddot{\text { hTDP-43 }}$

1. Rabbit IgG

2. hTREM2 Ab
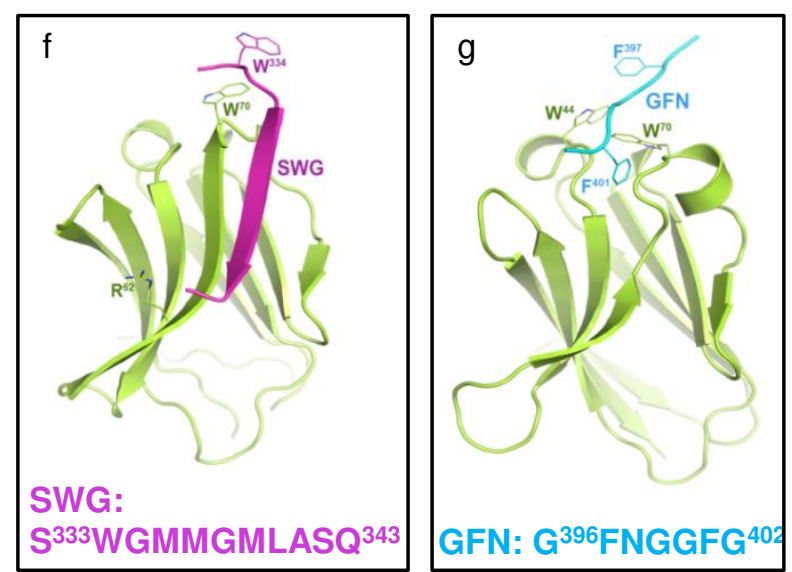

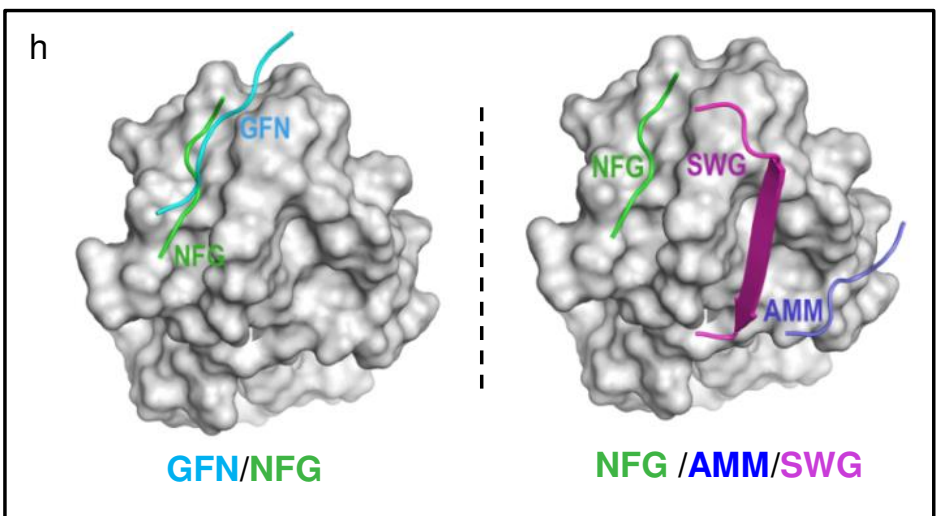



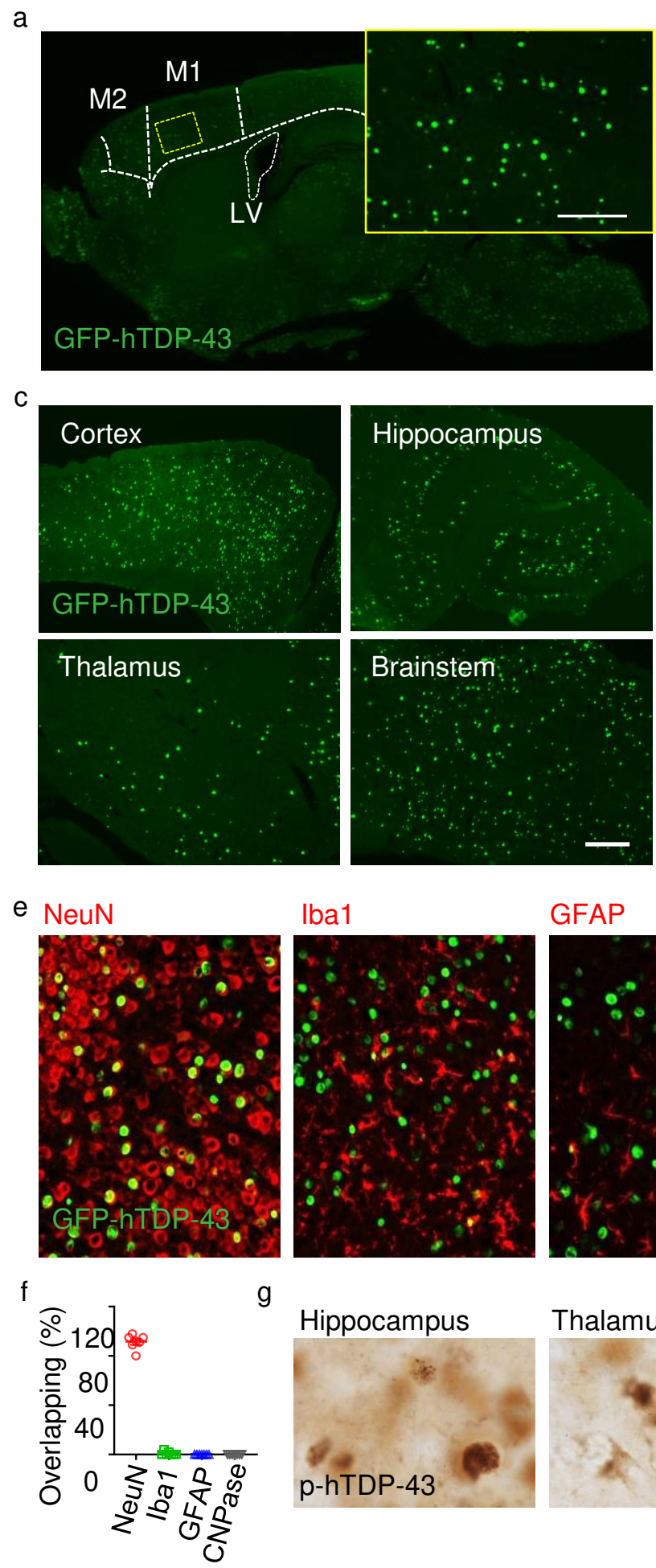

\begin{abstract}
Iba1
\end{abstract}
GFAP

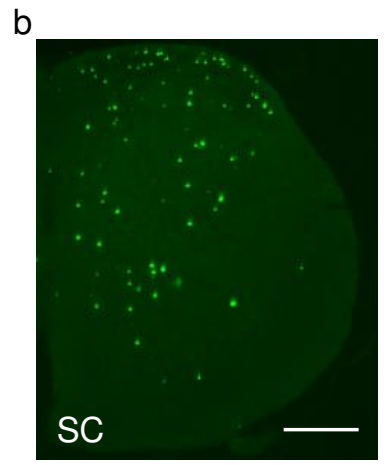

d
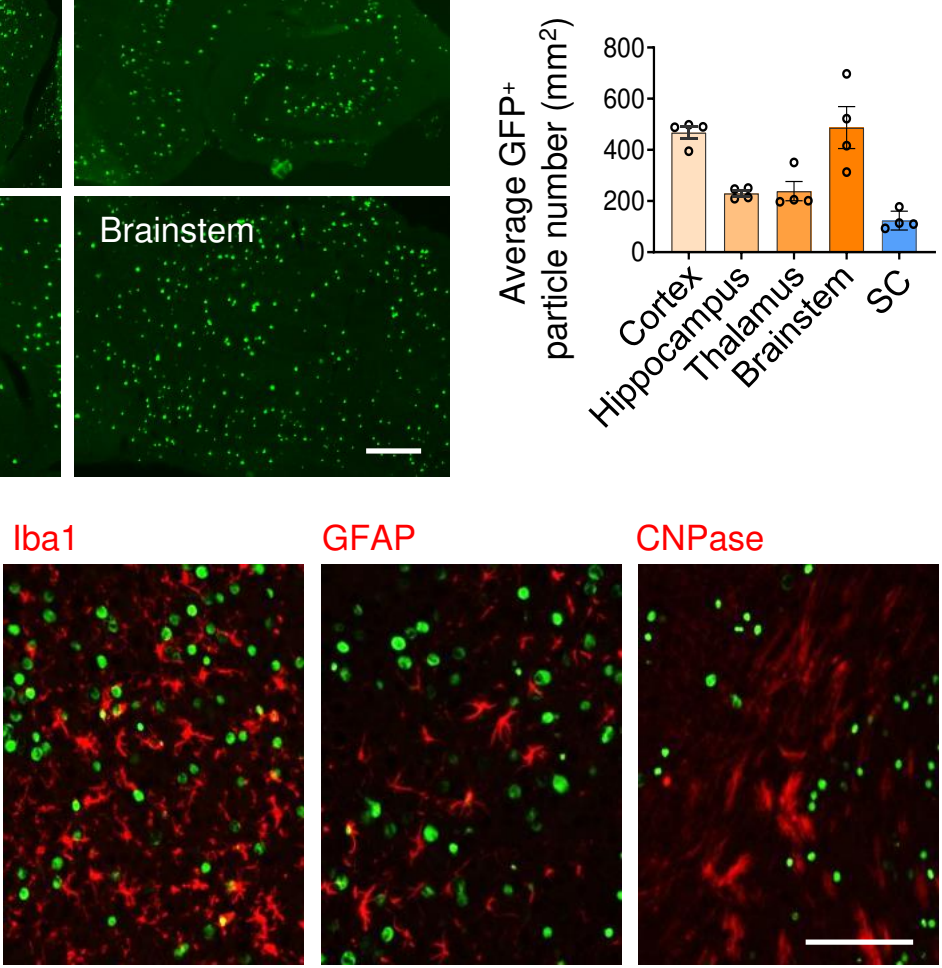

CNPase

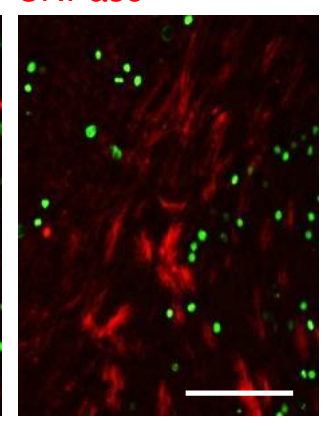

Thalamus

Brainstem

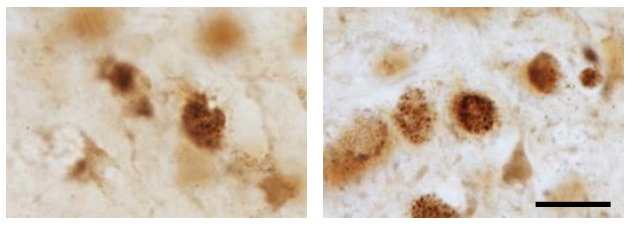


a

- Control $\Delta$ hTDP-43

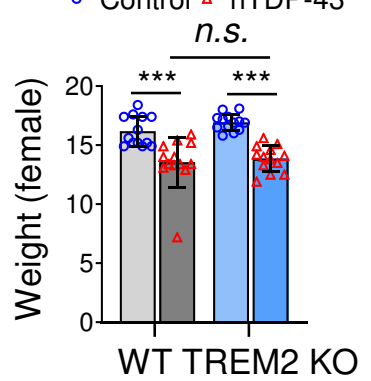

d
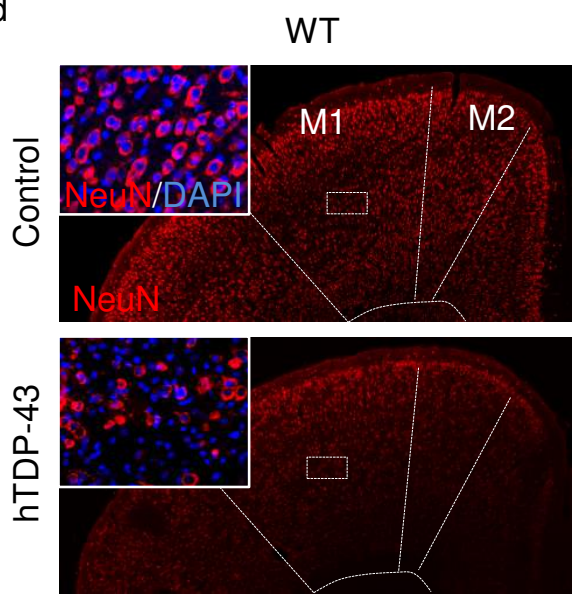

b

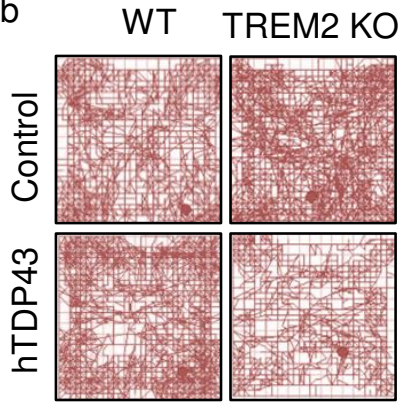

TREM2 KO
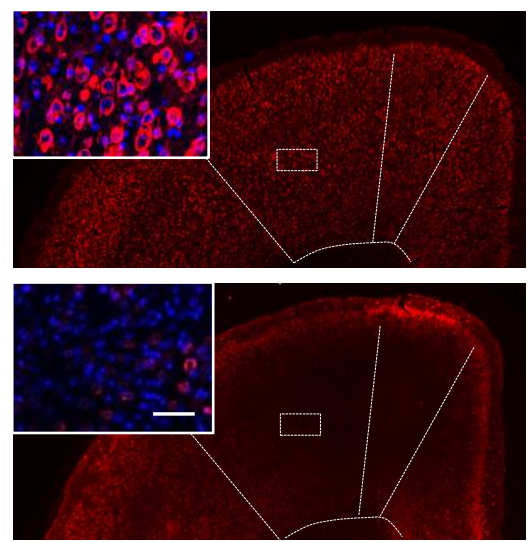

- Control a hTDP-43

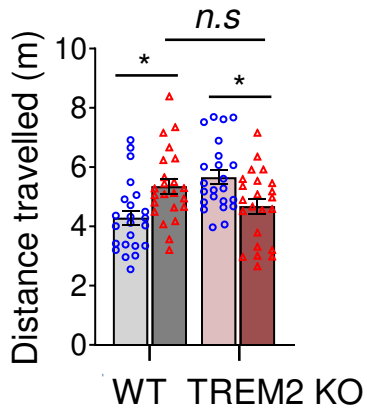

e

- Control

$\triangle$ hTDP-43

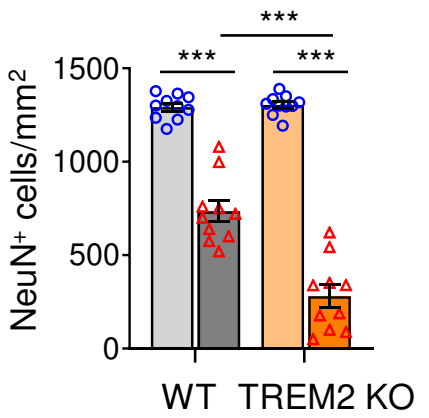




\section{Extended Data Figure 3}

a

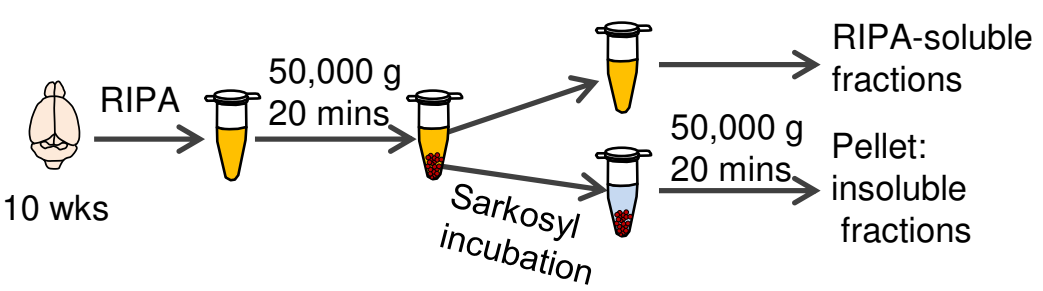

b

Soluble fractions
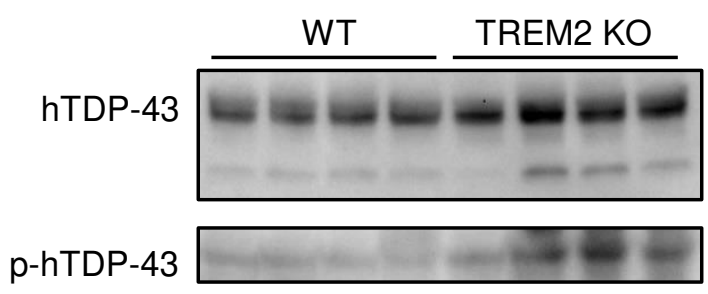

GAPDH $=m-m$

C

Insoluble fractions

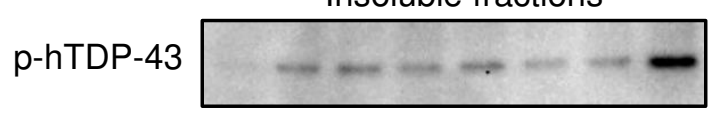


a
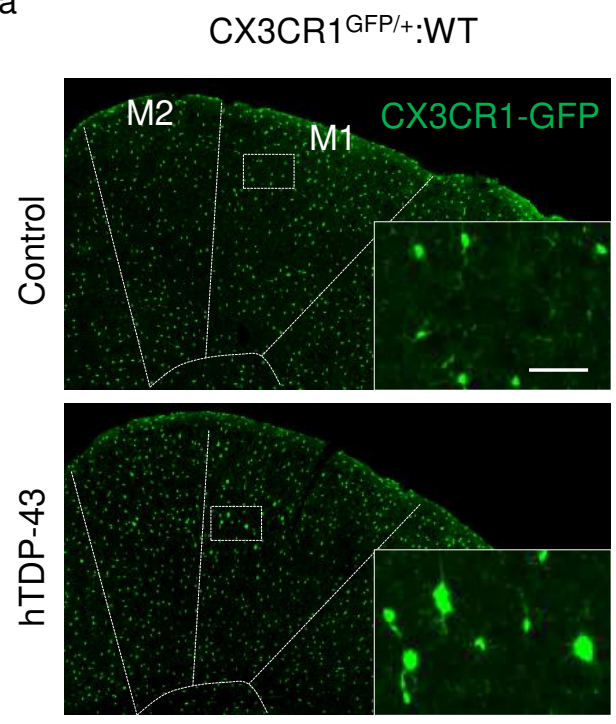

d

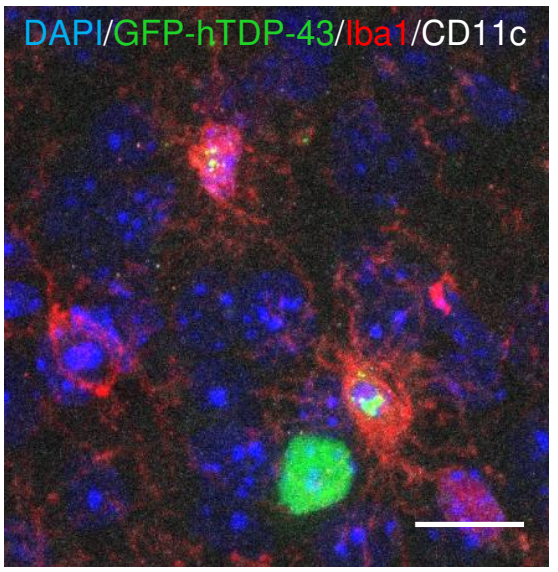

CX3CR1 $1^{\mathrm{GFP} /+}$ :TREM2 KO
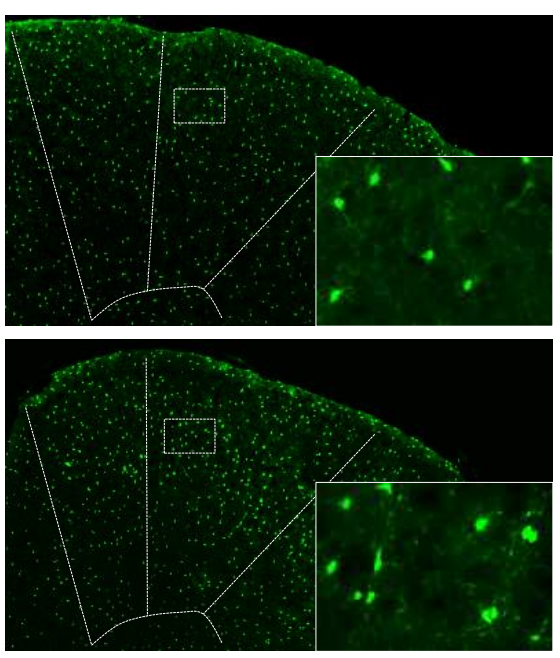

s.
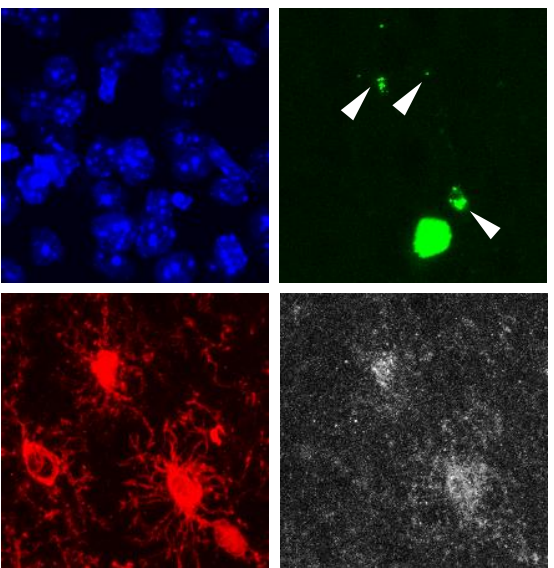

b

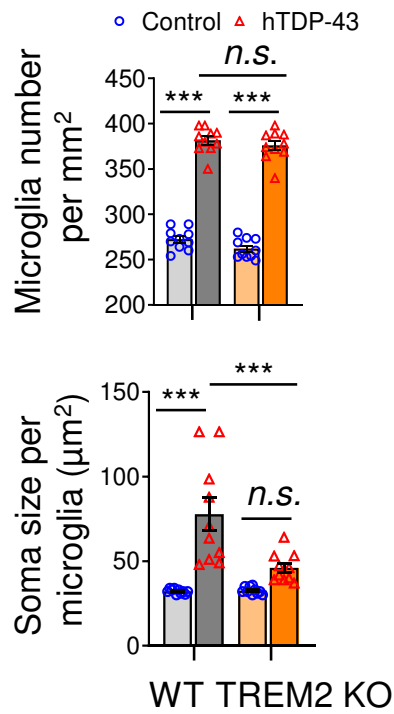

e

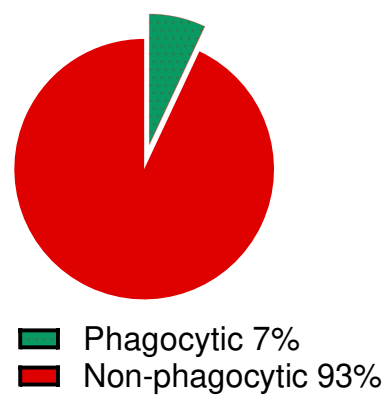


a
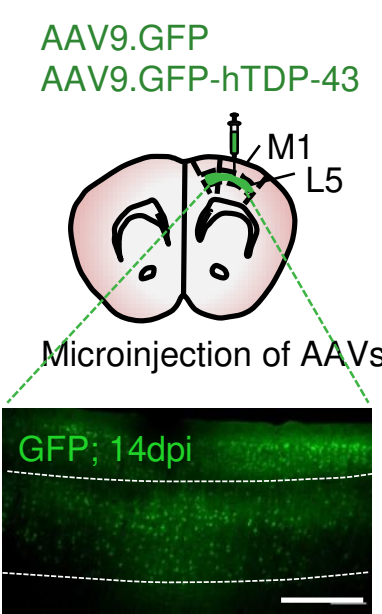

e

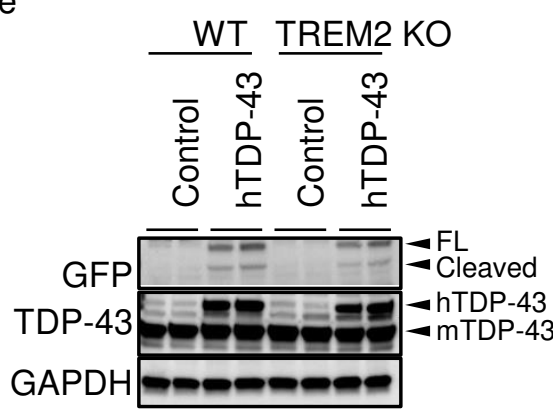

h

WT

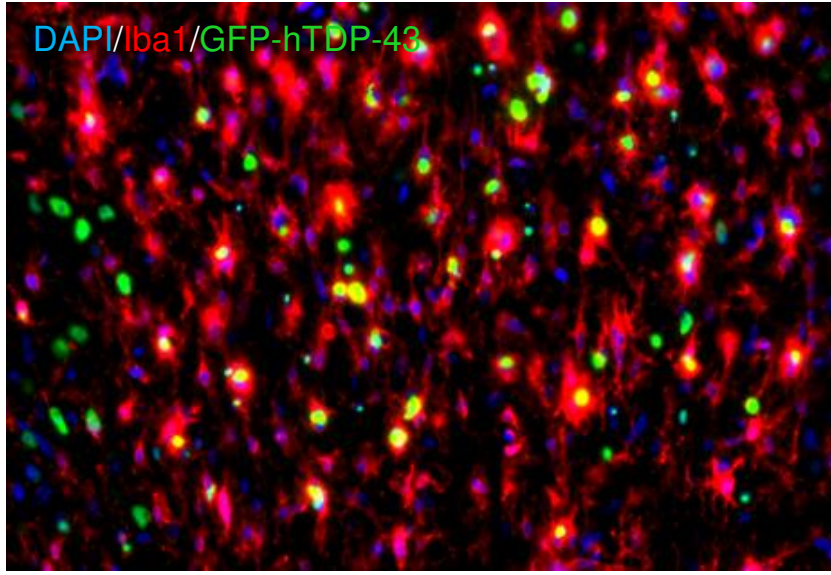

C

Mouse cortex

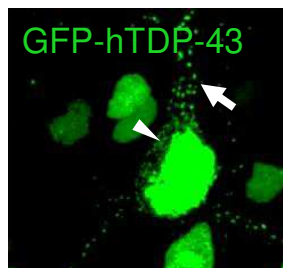

GFP-hTDP̂:-43

tdTomalo $\mathbf{k}$
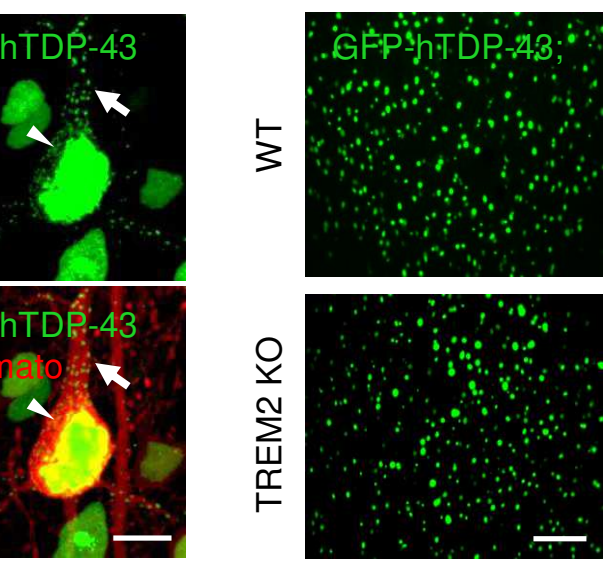

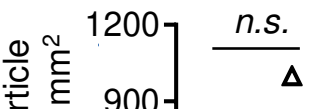

즌

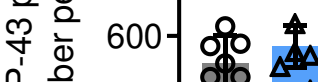

吕 点 $300-1$ 号

- WT

$\Delta$ TREM2 KO
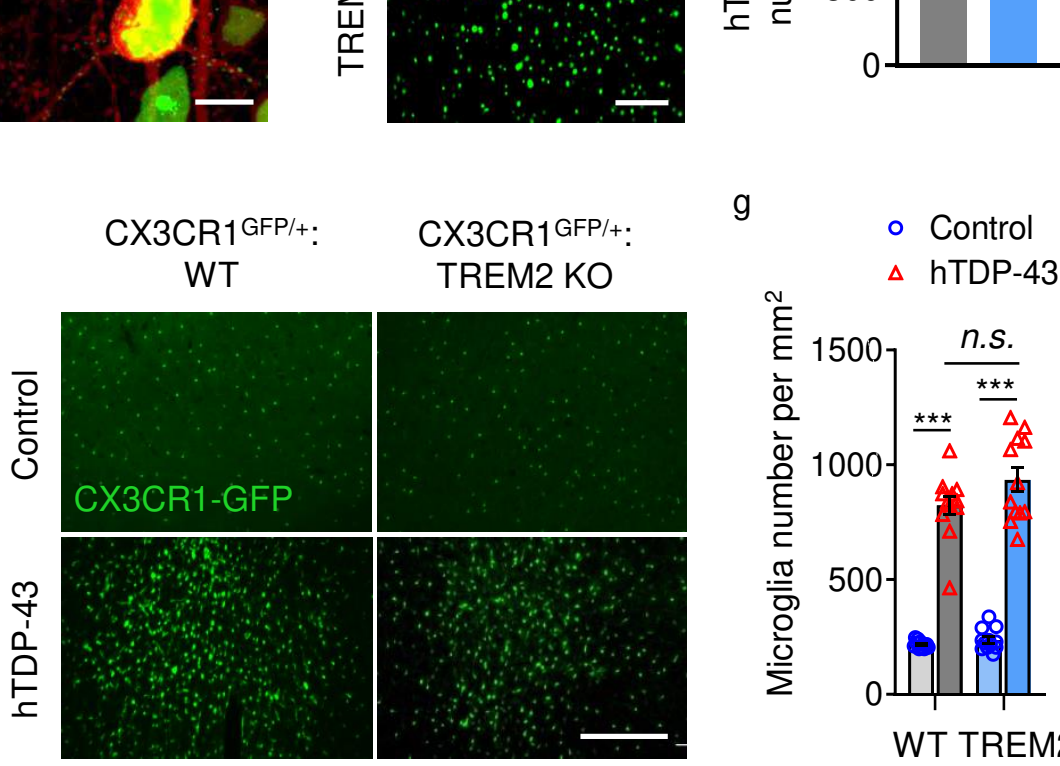

g
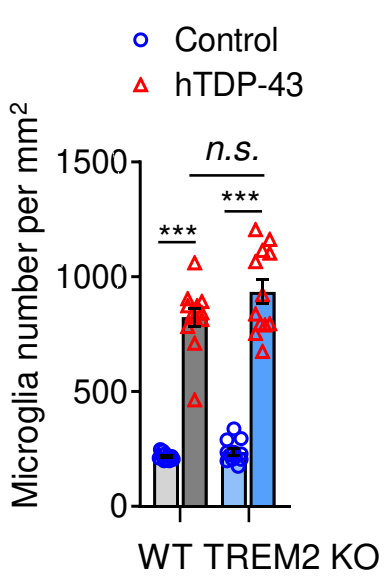

TREM2 KO

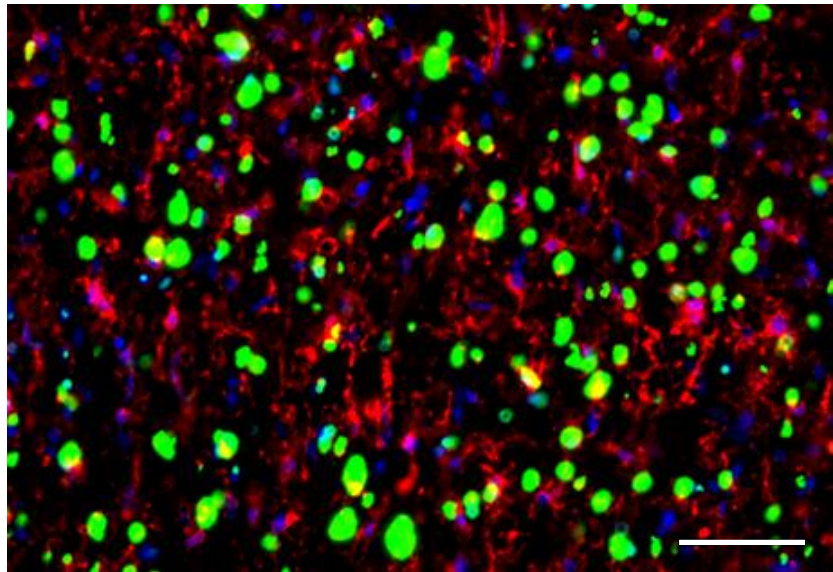


a

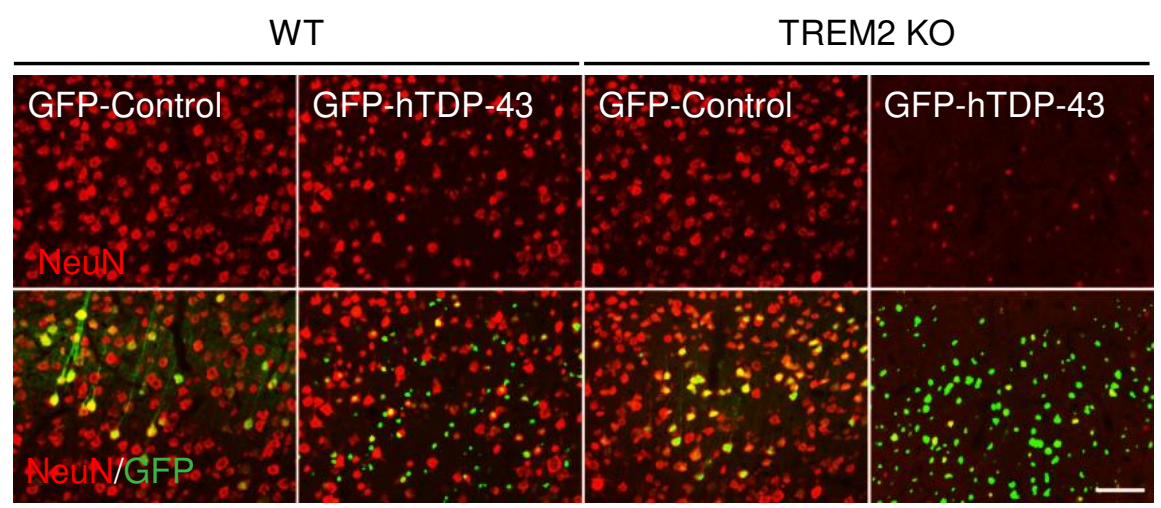

b

- Control

$\triangle$ hTDP-43

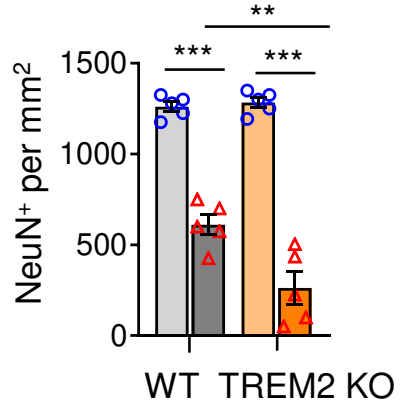

C

CX3CR1 $1^{\mathrm{GFP} /+}$ :
WT

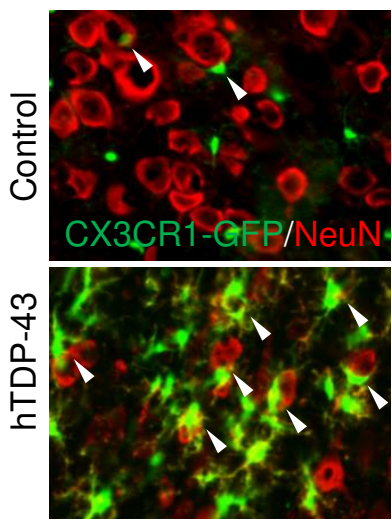

e

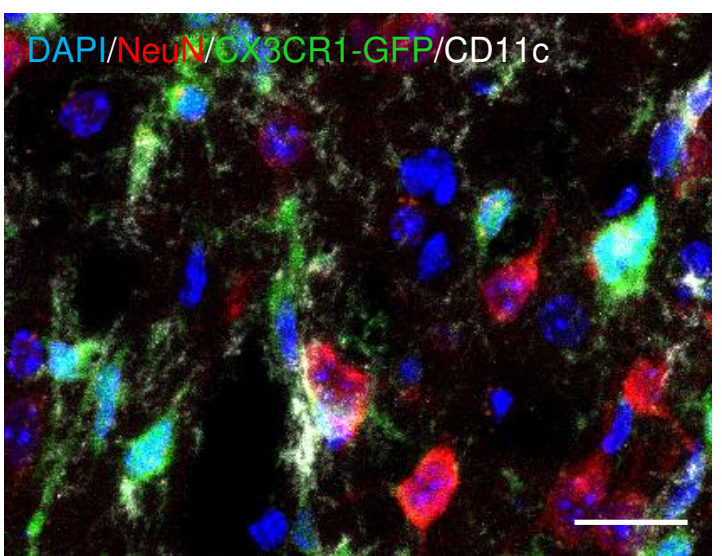

d

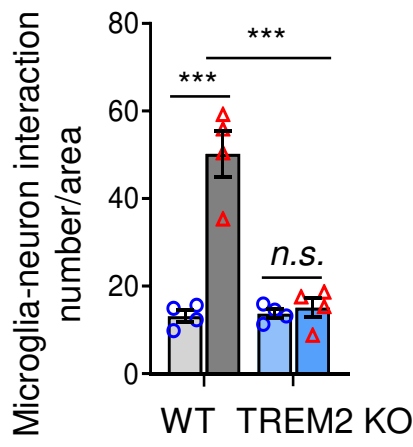

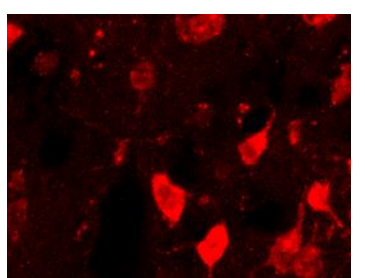
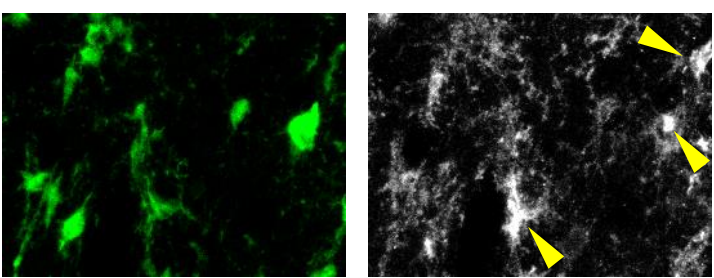
a

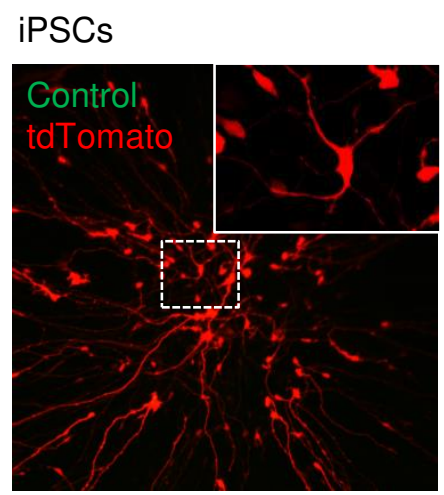

C
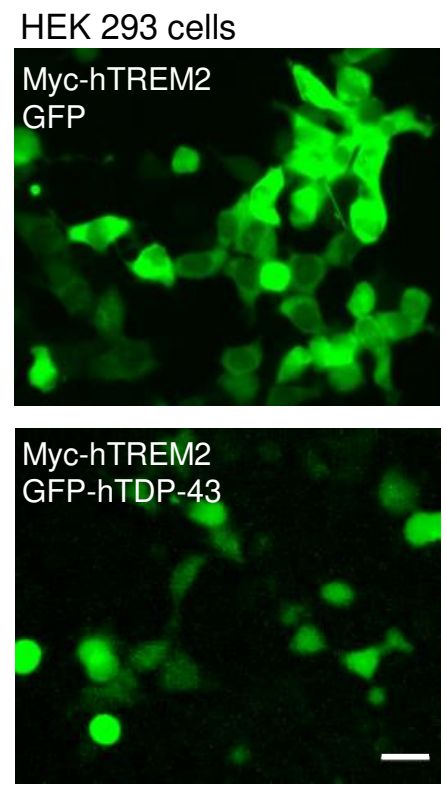

b

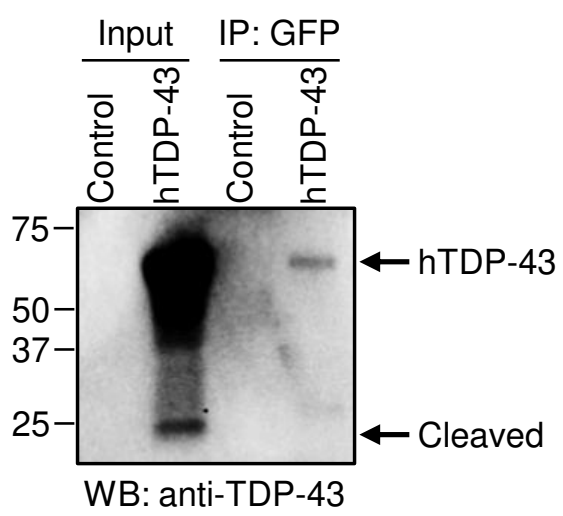

d
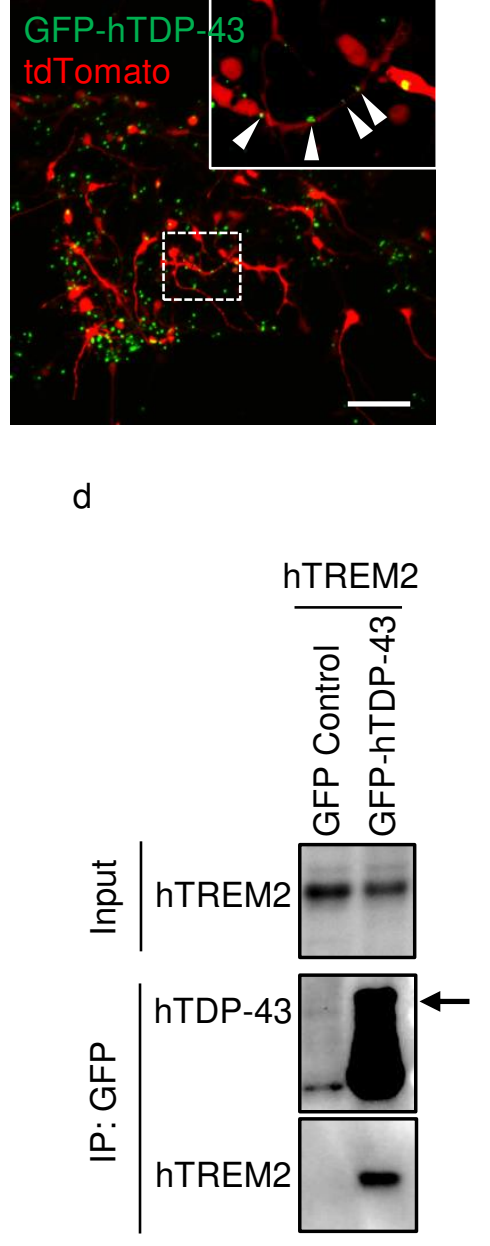

e

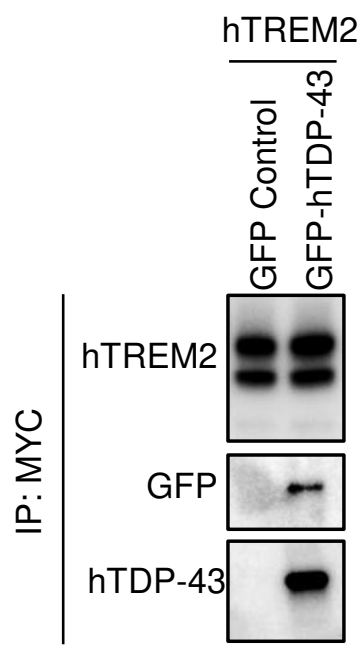


a

Soluble fractions
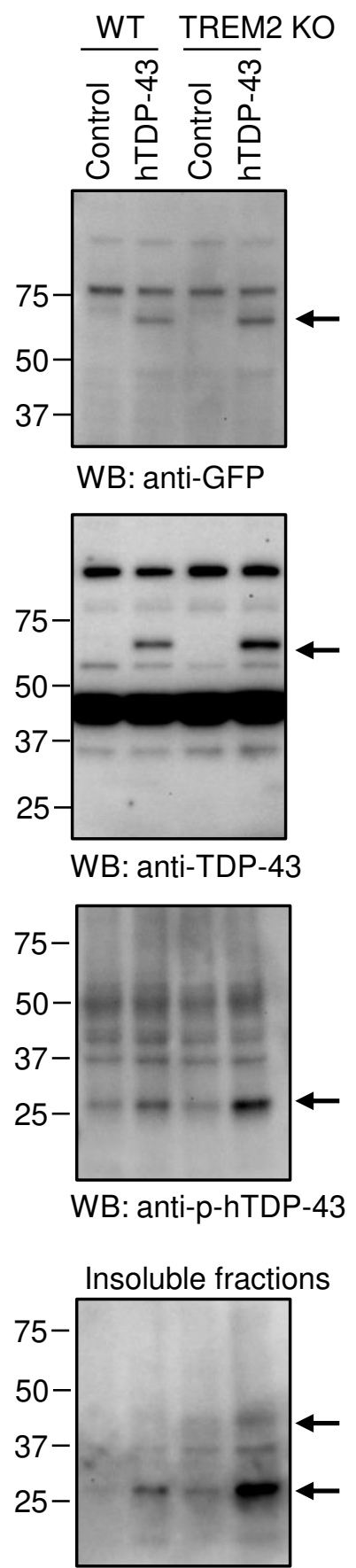

WB: anti-p-hTDP-43 b

HEK293 cell

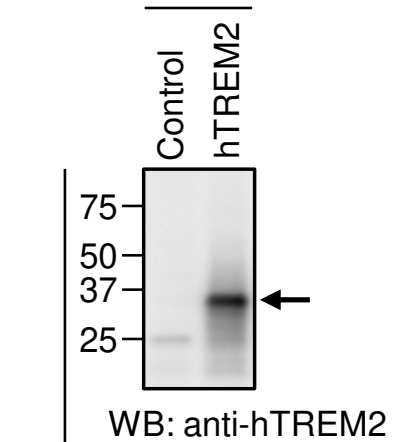

흘
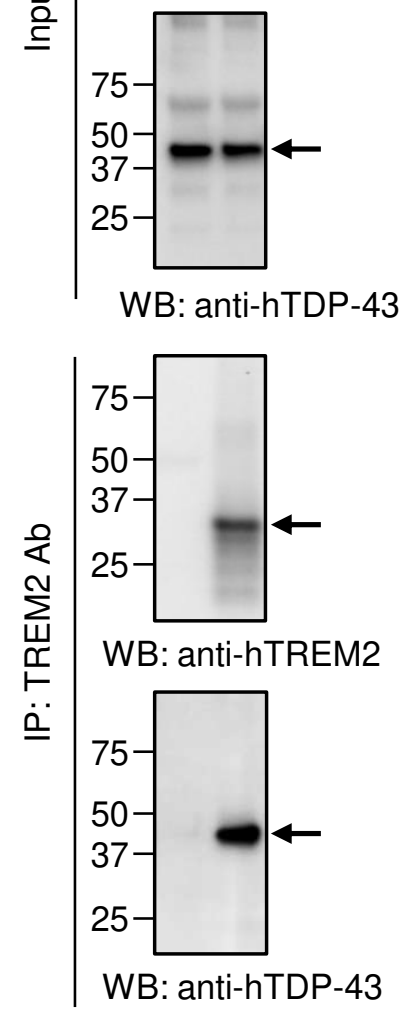

C Control rNLS
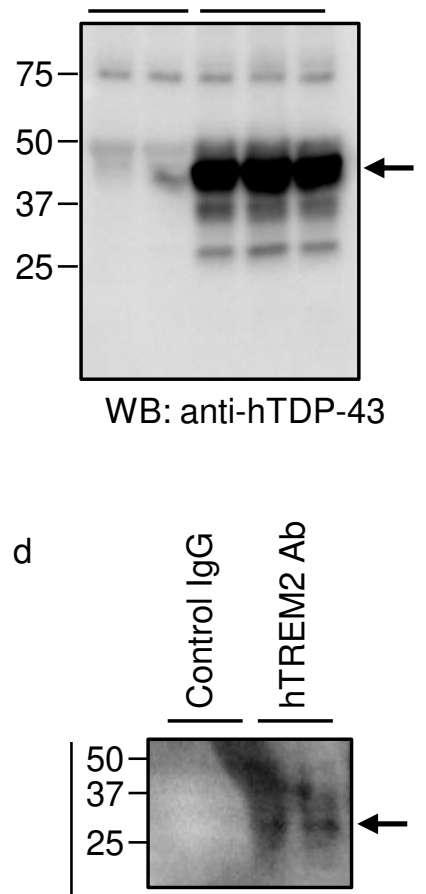

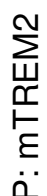

WB: anti-mTREM2 5F4 Nt

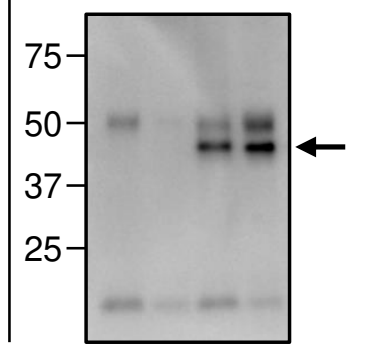

WB: anti-hTDP-43 


\section{Extended Data Figure 9}

a
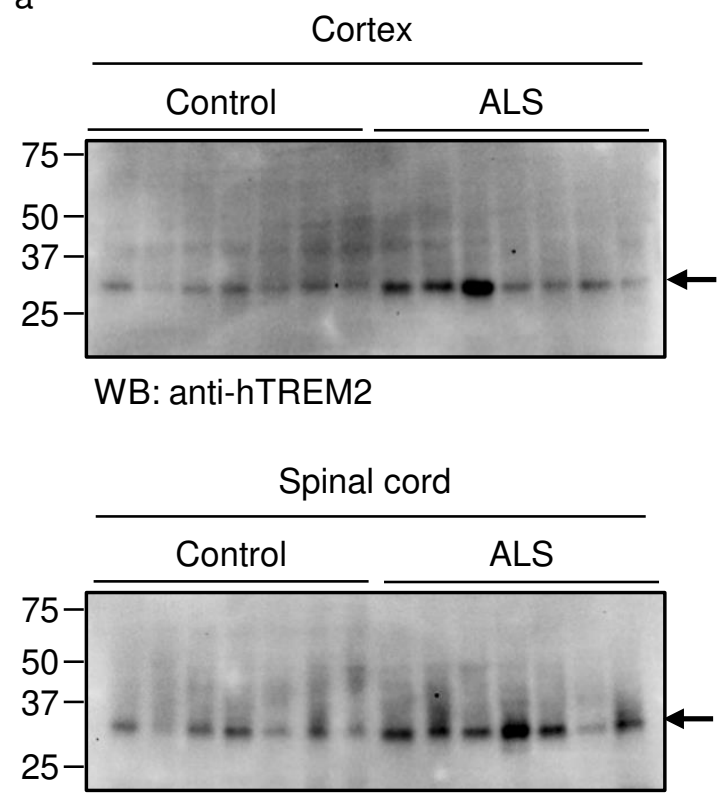

WB: anti-hTREM2

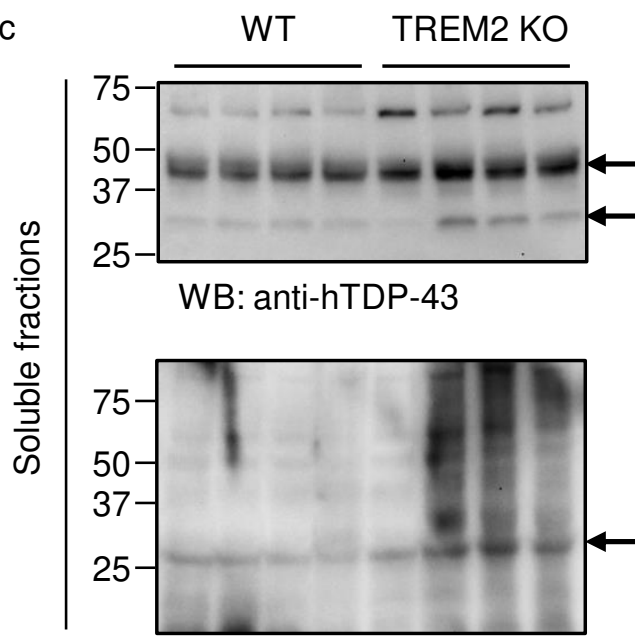

WB: anti-p-hTDP-43

d

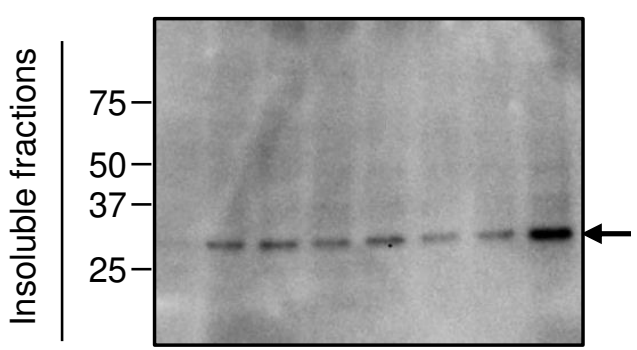

WB: anti-p-hTDP-43
Spinal cord (IP)

Control ALS

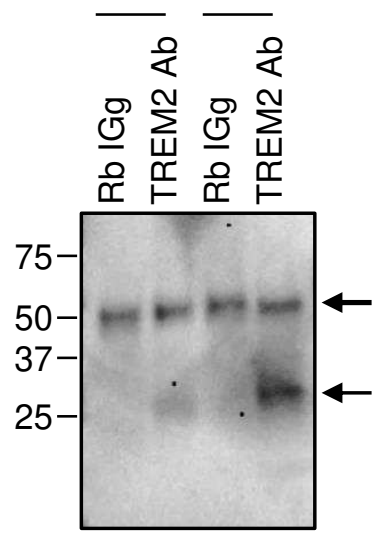

WB: anti-hTREM2

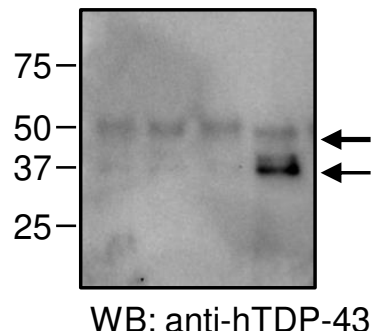

e

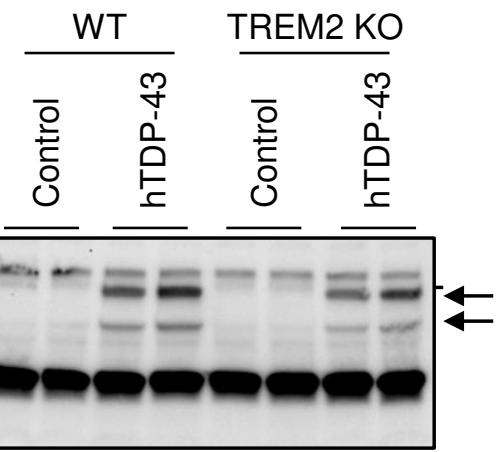

WB: anti-GFP

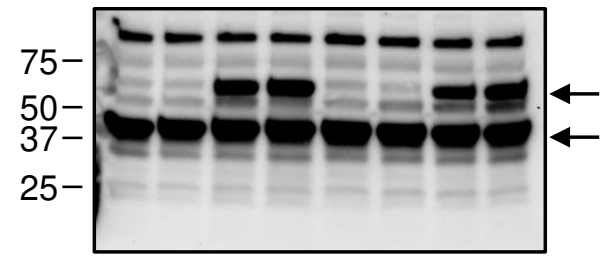

WB: anti-TDP-43 

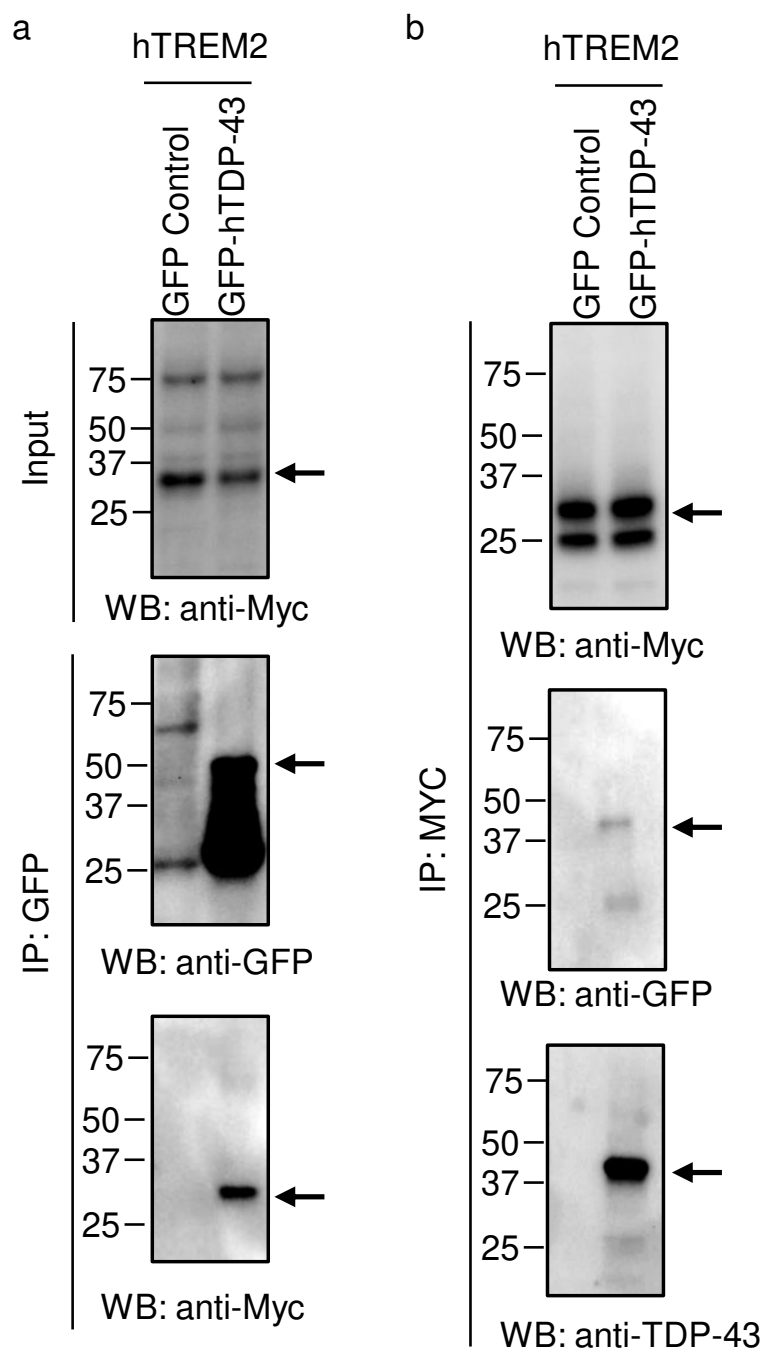
Supplementary Table1. Antibodies for CyTOF.

\begin{tabular}{|c|c|c|}
\hline Antibodies & Source & Identifier \\
\hline anti-mouse CCR2, 156Gd, monoclonal & Novus & $\begin{array}{l}\text { Cat\# MAB55381-100; } \\
\text { RRID:AB 2749828 }\end{array}$ \\
\hline anti-mouse CD45, 89Y, monoclonal & FDM & $\begin{array}{l}\text { Cat\# 3089005B; } \\
\text { RRID:AB_2651152 }\end{array}$ \\
\hline anti-mouse CD11c, $142 \mathrm{Nd}$, monoclonal & FDM & $\begin{array}{l}\text { Cat\# 3142003B; } \\
\text { RRID:AB 2814737 }\end{array}$ \\
\hline anti-mouse CD11b (Mac-1), 171Yb, monoclonal & FDM & $\begin{array}{l}\text { Cat\# 3143015B; } \\
\text { RRID:AB } 2811240\end{array}$ \\
\hline anti-mouse I-A/I-E, 209Bi, monoclonal & Biolegend & $\begin{array}{l}\text { Cat\# 107637; } \\
\text { RRID:AB_2563771 }\end{array}$ \\
\hline anti-mouse CD86, 151Eu, monoclonal & Biolegend & $\begin{array}{l}\text { Cat\# 105001; } \\
\text { RRID:AB 313144 }\end{array}$ \\
\hline anti-mouse MERTK, 155Gd, monoclonal & R\&D & $\begin{array}{l}\text { Cat\# MAB5912-100 } \\
\text { RRID:AB_2832245 }\end{array}$ \\
\hline anti-mouse F4/80, 159Tb, monoclonal & FDM & $\begin{array}{l}\text { Cat\# 3159009B; } \\
\text { RRID:AB 2811238 }\end{array}$ \\
\hline anti-mouse CD64, 160Gd, monoclonal & R\&D & $\begin{array}{l}\text { Cat\# MAB' } 20741 ; \\
\text { RRID:AB } 2246880\end{array}$ \\
\hline anti-mouse Ly6G, 161Dy, monoclonal & Biolegend & $\begin{array}{l}\text { Cat\# 127637; } \\
\text { RRID:AB_2563784 }\end{array}$ \\
\hline anti-mouse SiglecH, 163Dy, monoclonal & Biolegend & $\begin{array}{l}\text { Cat\# 129602; } \\
\text { RRID:AB 1227757 }\end{array}$ \\
\hline anti-mouse CX3CR1, 164Dy, monoclonal & FDM & $\begin{array}{l}\text { Cat\# 3164023B; } \\
\text { RRID:AB 2832247 }\end{array}$ \\
\hline anti-mouse CD206 (MMR), 169Tm, monoclonal & FDM & $\begin{array}{l}\text { Cat\# 3169021B; } \\
\text { RRID:AB 2832249 }\end{array}$ \\
\hline anti-mouse CD115/CSF1R, $174 \mathrm{Yb}$, monoclonal & Biolegend & $\begin{array}{l}\text { Cat\# 135521; } \\
\text { RRID:AB 2563709 }\end{array}$ \\
\hline anti-mouse Ly6C, 175Lu, monoclonal & Biolegend & $\begin{array}{l}\text { Cat\# 128039; } \\
\text { RRID:AB_2563783 }\end{array}$ \\
\hline
\end{tabular}


Supplementary Table2. Basic characteristics of human tissue donors.

\begin{tabular}{|c|c|c|c|c|c|}
\hline Mayo ID & TDP-43 & Thal phase & Duration(year) & Age & Sex \\
\hline ALS1 & 1 & 3 & 5 & 53 & $\mathrm{~F}$ \\
\hline ALS2 & 1 & 0 & 2 & 60 & $\mathrm{M}$ \\
\hline ALS3 & 1 & 0 & 2 & 59 & $F$ \\
\hline ALS4 & 1 & 0 & 3 & 58 & $\mathrm{~F}$ \\
\hline ALS5 & 1 & 0 & 5 & 60 & $\mathrm{~F}$ \\
\hline ALS6 & 1 & 0 & 7 & 64 & $\mathrm{M}$ \\
\hline ALS7 & 1 & 0 & 1 & 72 & $F$ \\
\hline ALS8 & 1 & 1 & 3 & 65 & $\mathrm{~F}$ \\
\hline ALS9 & 1 & 1 & 4 & 62 & $\mathrm{~F}$ \\
\hline ALS10 & 1 & 4 & 3 & 60 & $F$ \\
\hline ALS11 & 1 & 0 & NA & 64 & $\mathrm{~F}$ \\
\hline ALS12 & 1 & 2 & NA & 75 & $\mathrm{M}$ \\
\hline ALS13 & 1 & 3 & NA & 78 & $\mathrm{M}$ \\
\hline ALS14 & 1 & 2 & NA & 79 & $F$ \\
\hline ALS15 & 1 & 1 & NA & 80 & $\mathrm{M}$ \\
\hline ALS16 & 1 & 3 & NA & 83 & $\mathrm{~F}$ \\
\hline Control 1 & 0 & 0 & NA & 64 & $\mathrm{M}$ \\
\hline Control 2 & 0 & 3 & NA & 75 & $\mathrm{M}$ \\
\hline Control 3 & 0 & 3 & NA & 85 & $\mathrm{M}$ \\
\hline Control 4 & 0 & 0 & NA & 77 & $\mathrm{M}$ \\
\hline Control 5 & 0 & 0 & NA & 92 & $F$ \\
\hline Control 6 & 0 & 0 & NA & 83 & $\mathrm{~F}$ \\
\hline Control 7 & 0 & 0 & NA & 67 & M \\
\hline Control 8 & 0 & 0 & NA & 79 & $F$ \\
\hline Control 9 & 0 & 0 & NA & 80 & $\mathrm{~F}$ \\
\hline Control 10 & 0 & 0 & NA & 54 & $\mathrm{~F}$ \\
\hline Control 11 & 0 & 0 & NA & 76 & $M$ \\
\hline Control 12 & 1 & 0 & NA & 64 & $\mathrm{~F}$ \\
\hline
\end{tabular}

ALS: amyotrophic lateral sclerosis 
a

Survival rate

Hindlimb clasping score

$\longrightarrow$

$\mathrm{ICV}$ injection on $\mathrm{PO}$
Neuronal loss

TDP-43 pathology

Microglia response b

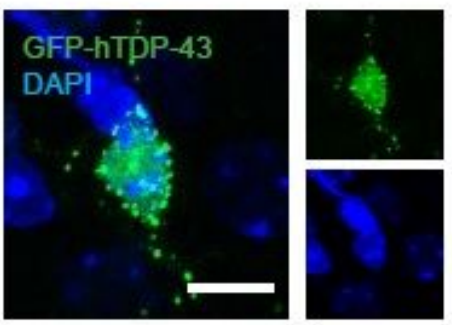

c

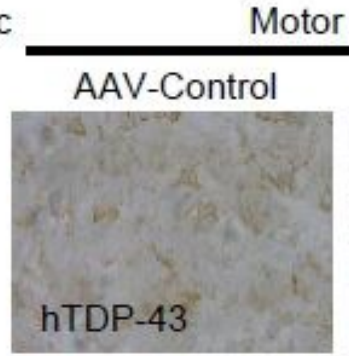

Motor cortex

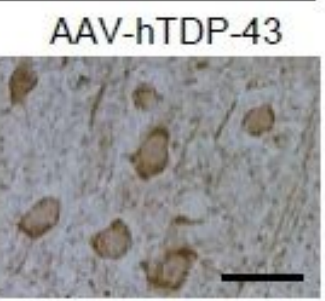

d

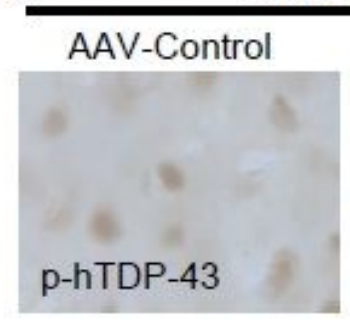

Rotarod test

5 weeks

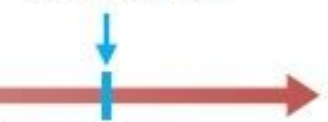

10 weeks e

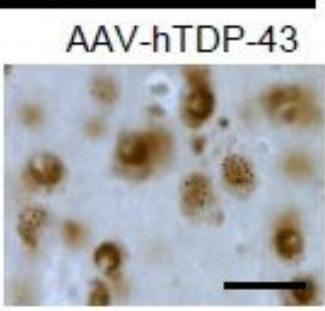

hTDP-43
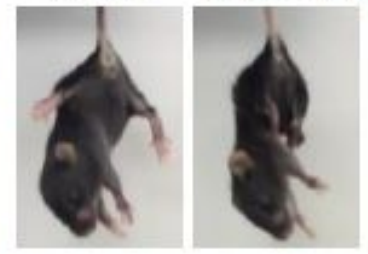

f
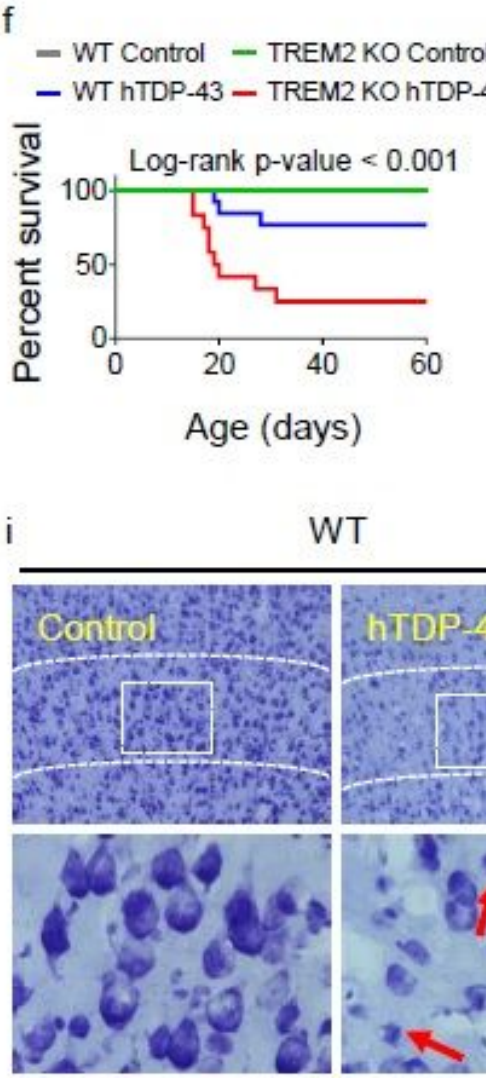

g

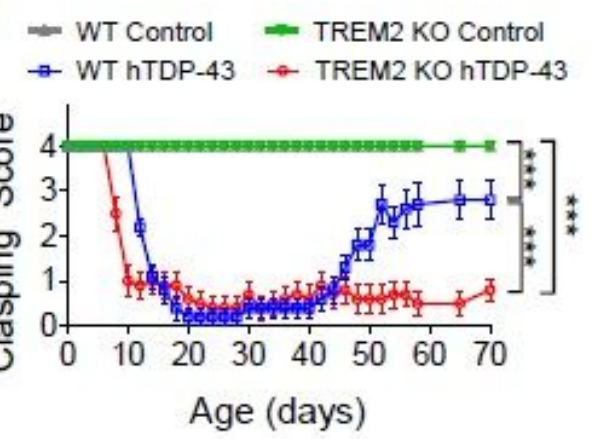

TREM2 KO

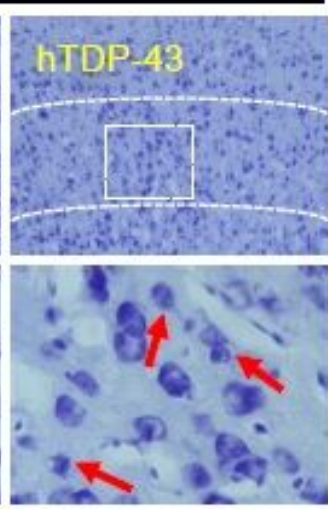

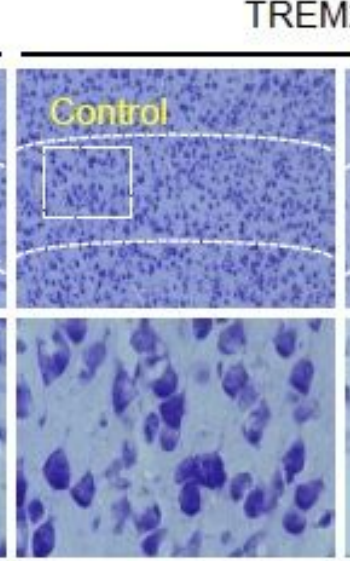

h

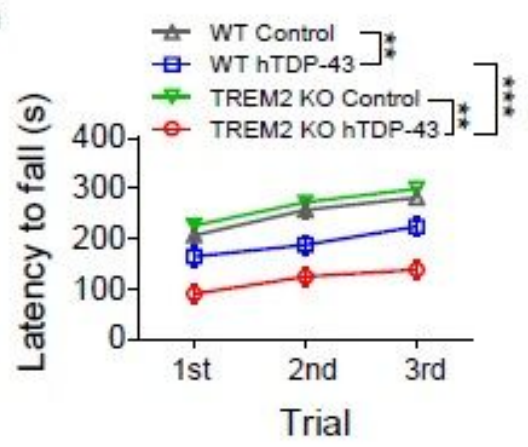

\section{Figure 1}

TREM2 deficiency aggravates hTDP-43-induced behavioral deficits and neurodegeneration. GFP tagged hTDP-43 protein (GFP-hTDP-43) expression was induced via intracerebroventricular injection of AAV9.CAG.hTDP43-GFP in neonatal mice (AAV9.CAG.GFP as control). a, Study design and timeline for 
the neonatal ICV injection model. b, Representative images of GFP-hTDP-43 expression in both nucleus and cytosol of neurons in the primary motor cortex of WT mice at 35 days post-infection (dpi); Scale bar, $10 \mu \mathrm{m}$. c-d, Representative images of hTDP-43 (c) or p-hTDP-43 (d) expression in the motor cortex at 35 dpi after AAV-control or AAV-hTDP-43 infection; Scale bar, $20 \mu \mathrm{m}$. e, Representative images of typical clasping phenotype in WT mice expressing hTDP-43 at $14 \mathrm{dpi}$. f, Kaplan-Meier survival curves show the percentage of mice alive at each postnatal day up to $60 \mathrm{dpi}$ ( $n=20$ per group). g, Hindlimb clasping response scores collected over 70 days ( $n=10$ per group). $h$, Average latency to fall during rotarod analysis at $70 \mathrm{dpi}$ ( $\mathrm{n}=10$ per group). i, Representative images of Nissl staining in the primary motor cortex of indicated groups at $35 \mathrm{dpi}$; Dashed lines indicate the borders of layer 4\&5; Scale bar, $100 \mu \mathrm{m}$; High magnification images as indicated by area in dotted white box showing neuronal morphology of the cortical layer $\mathrm{V}$ at the bottom; Scale bar, $50 \mu \mathrm{m}$. Red arrows indicate neuronal shrinkage and red arrowheads indicate a significant loss of Nissl staining. j, Quantification of neuron numbers in the primary motor cortex of indicated groups at $35 \mathrm{dpi}$ ( $n=10$ per group). Data represented as mean \pm SEM. Significance was calculated using two-way ANOVA followed by Tukey's post hoc test; n.s., not significant; $\star P<0.05,{ }^{*} \mathrm{P}<0.01, * \star \star \mathrm{P}<0.001$. 
a

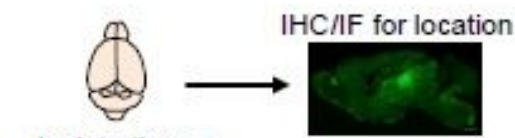

$\mathrm{CD} 45^{+} \mathrm{CD} 11 \mathrm{~b}^{+}$

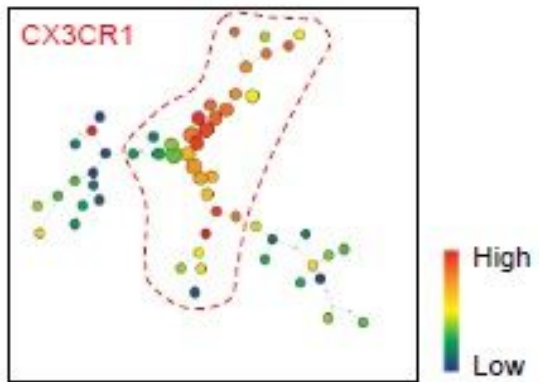

e

- Control

4 hTDP-43 b

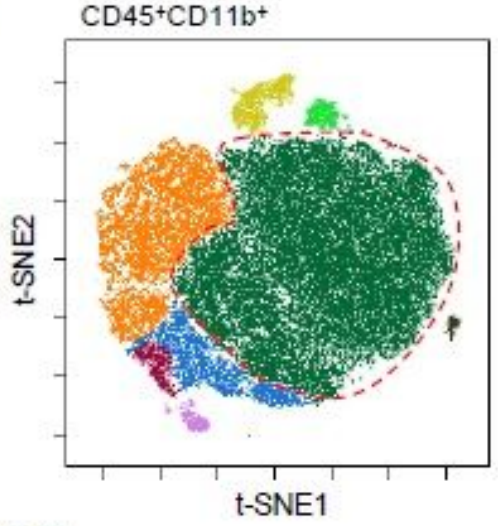

d

Microglia

CD45 CD11b CX3CR1 F4/80
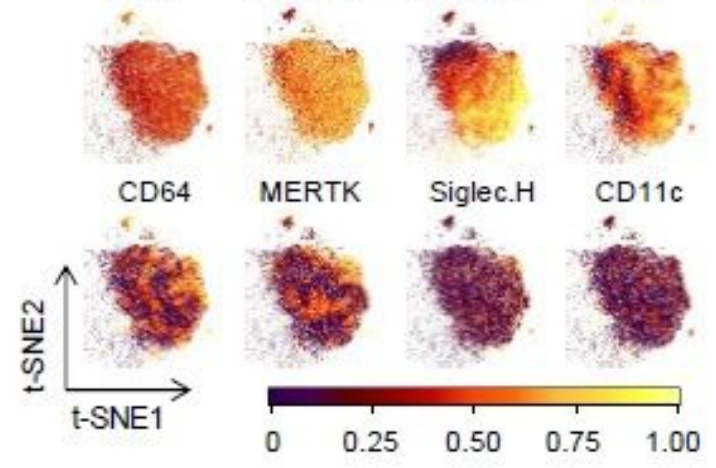

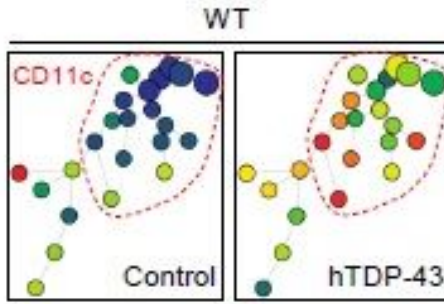

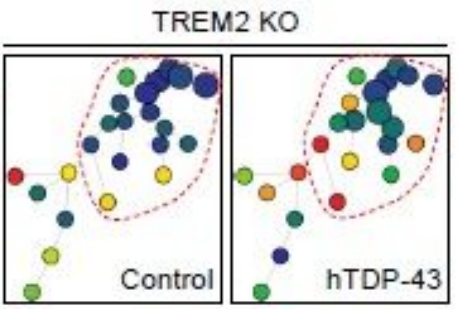

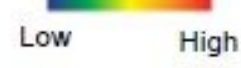

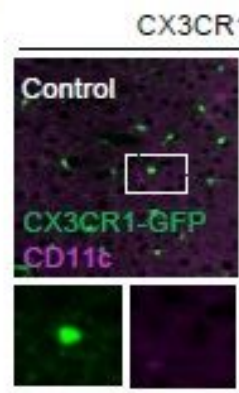

CX3CR $1^{\text {GFPl+:-TREM } 2 \text { KO }}$
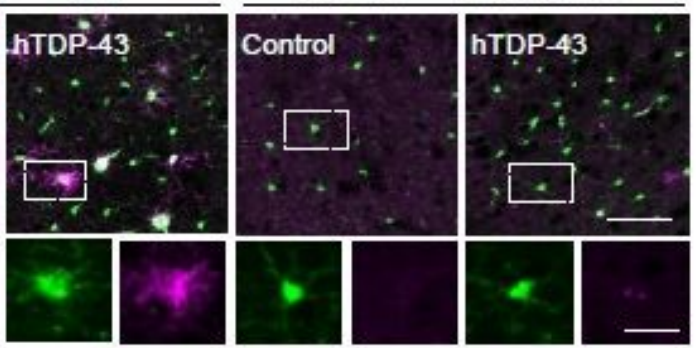

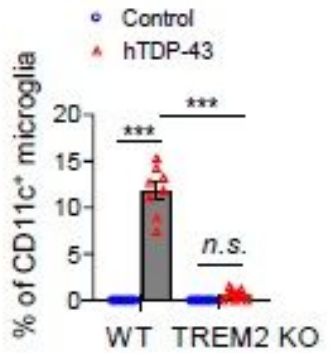

\section{Figure 2}

TREM2 deficiency abolishes hTDP-43-induced CD11c+ microglia subpopulation. hTDP-43 protein expression was induced via intracerebroventricular injection of AAV9.CAG.hTDP-43 in neonatal mice (AAV9.CAG.Empty as control). a, Schematic workflow for cytometry by time of flight mass spectrometry (CyTOF) and immunostaining. b, t-SNE map displaying CD45+CD11b+ sampled cells from the brain of steady-state 6 to 8-week-old WT mice analyzed by mass cytometry. Colors correspond to FlowSOM- 
guided clustering of cell populations ( $n=8$, representative of 4 independent experiments). c, Spanningtree progression analysis of density-normalized events (SPADE) on CD45+CD11b+ cell populations. The size of each spot is determined by the number of cells within this population. $d$, Microglia were plotted onto a t-SNE. Plots represent distinguishing cell surface markers for microglia of 6-8 week-old WT mice. Clustering analysis revealed a major microglia population characterized by CD45mid:CD11b+:CX3CR1hi:F4/80+:CD64+:MeTK+:Siglec-H+:CD11c-. e, Frequency analysis of microglia based on manual gating of indicated groups at $35 \mathrm{dpi}(n=8$, representative of 4 independent experiments). Increased number of microglia was observed in both WT and TREM2 KO mice expressing hTDP-43. $\mathrm{f}$, SPADE reveals a unique CD11c+ microglia sub-population in WT mice expressing hTDP-43 at $35 \mathrm{dpi}$. In hTDP expressing TREM2 KO mice, CD11c+ microglia sub-population was largely abolished. The size of each spot is determined by the number of cells within this population. The color code shows the expression level of $C D 11 \mathrm{c}$. g, Representative images of CD11c (purple) expression by immunostaining in the primary motor cortex of indicated groups at $35 \mathrm{dpi}$; Scale bar, $100 \mu \mathrm{m}$. High magnification images as indicated by area in dotted white box showing the single microglia at the bottom; Scale bar, $10 \mu \mathrm{m} . \mathrm{h}$, Average frequency of microglia expressing CD11 $\mathrm{c}$ in the primary motor cortex of indicated groups at 35 dpi ( $n=8$ per group). CD11 c microglia subpopulation was present in the WT mice expressing hTDP-43; however, few in TREM2 KO mice expressing hTDP-43 were observed. Data represented as mean \pm SEM. Significance was calculated using two-way ANOVA followed by Tukey's post hoc test; n.s., not significant; $\star P<0.05,{ }^{\star} * \mathrm{P}<0.01, \star \star \star \mathrm{P}<0.001$. 


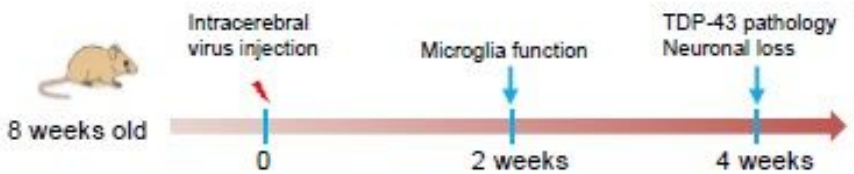

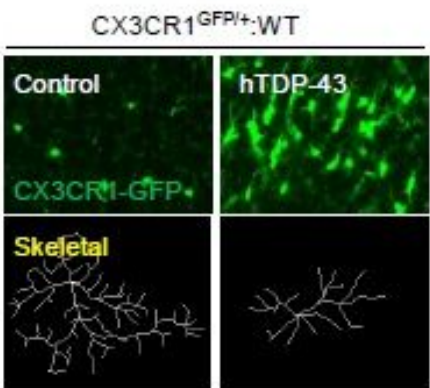

CX3CR $1^{\text {GFPlt:TREM } 2 \mathrm{KO}}$
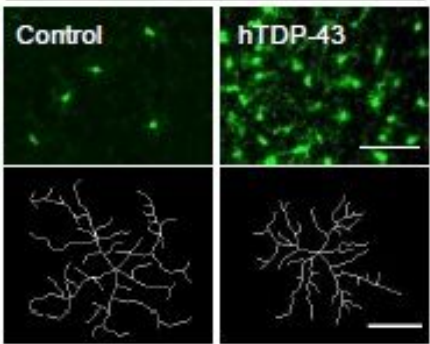

f

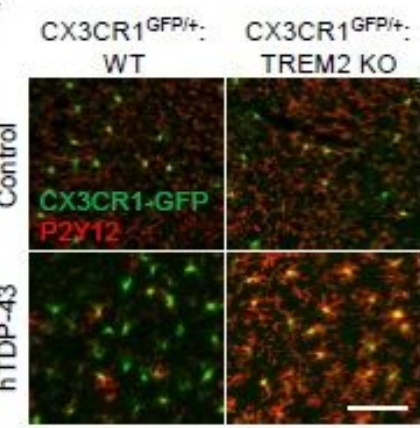

j
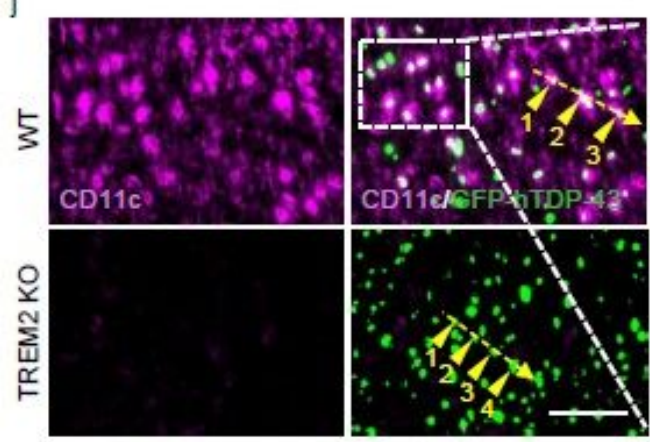

k

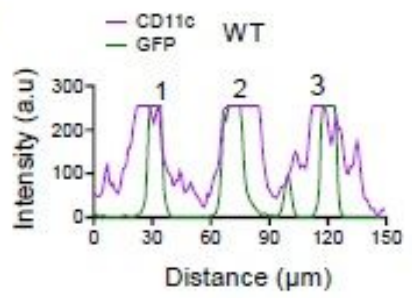

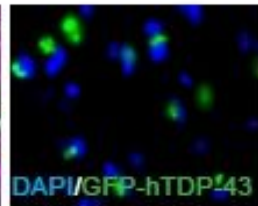

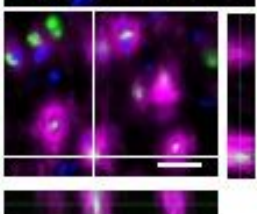

$9 \mathrm{CX} 3 \mathrm{CR}^{\mathrm{GFP} / \mathrm{t}}: \mathrm{CX} 3 \mathrm{CR} 1^{\mathrm{GPP} i t}$.
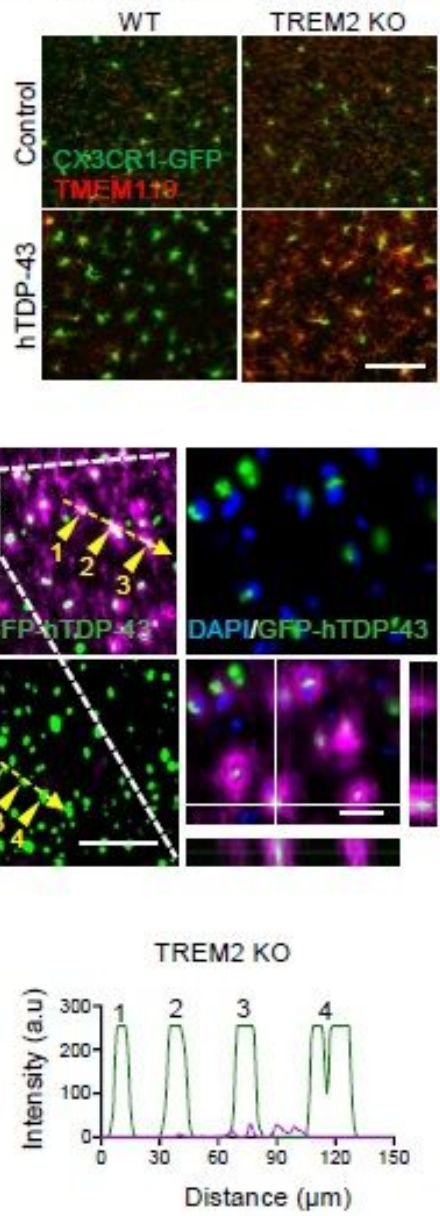
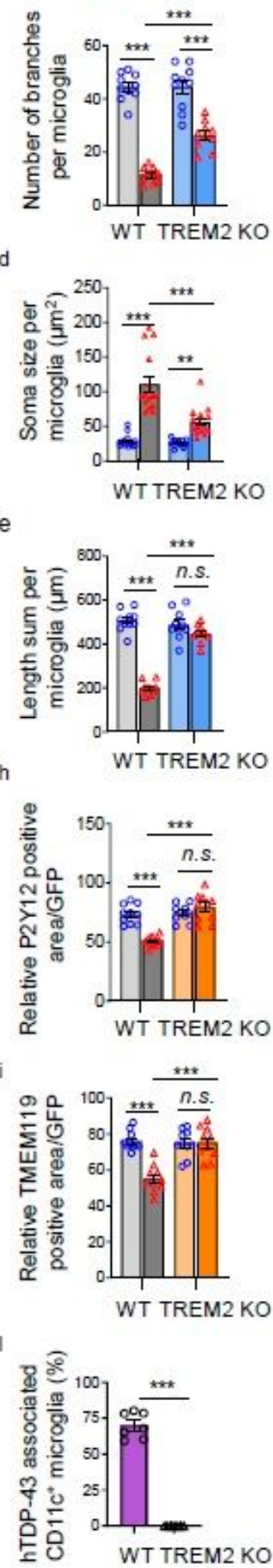

\section{Figure 3}

TREM2 deficiency locks microglia into a homeostatic state in TDP-43-induced neurodegeneration. In adult local injection model, hTDP-43 or GFP-hTDP-43 was expressed in the primary motor cortex of 2month-old mice via stereotactic intracerebral injection of AAV9.CAG.hTDP-43 or AAV9.CAG.hTDP43-GFP (AAV9.CAG.Empty or AAV9.CAG.GFP as control). a, Study design and timeline for local hTDP-43 expression model. b, Representative images (upper panels; Scale bar, $100 \mu \mathrm{m}$ ) and transformed skeletal 
images (lower panels; Scale bar, $10 \mu \mathrm{m}$ ) of GFP-expressing microglia in the primary motor cortex of indicated groups at $14 \mathrm{dpi}$. c-e, Quantification of GFP-expressing microglia number of branches (c, n=10 per group), soma size ( $d, n=15$ per group) and process length (e, $n=10$ per group) in the primary motor cortex of indicated groups at $14 \mathrm{dpi}$. WT microglia displayed a reactive phenotype as reduced branches, increased soma size and shorter processes in response to hTDP-43 expression. However, this hTDP-43induced reactive phenotype was significantly attenuated in TREM2 KO mice. $\mathrm{f}$, Representative images of P2Y12 expression (red) in the primary motor cortex of indicated groups at $7 \mathrm{dpi}$; Scale bar, $100 \mu \mathrm{m} . \mathrm{g}$, Representative images of TMEM119 expression (red) in the primary motor cortex of indicated groups at 7 dpi; Scale bar, $100 \mu \mathrm{m}$. h, i, Quantification of relative P2Y12 and TMEM119 positive area in the primary motor cortex of indicated groups at $7 \mathrm{dpi}(\mathrm{n}=10$ per group). j, Representative images of CD11c (purple), hTDP-43 (green), and DAPI staining (blue) in the primary motor cortex of indicated groups at $28 \mathrm{dpi}$. Scale bar, $100 \mu \mathrm{m}$. High magnification images as indicated by area in dotted white box showing on the right; Scale bar, $10 \mu \mathrm{m}$. k, Analysis of co-localization of CD11c (purple curves) with GFP-hTDP-43 (green curves) in the primary motor cortex of indicated groups at $28 \mathrm{dpi}$. Fluorescence intensity profiles of CD11c and GFP-hTDP-43 show the distribution of fluorescence across the yellow dotted arrows in (j). I, Average frequency of hTDP-43 associated CD11c+ microglia in the primary motor cortex of indicated groups at $28 \mathrm{dpi}(\mathrm{n}=7$ per group). Results indicate that $\mathrm{CD} 11 \mathrm{c}+$ microglia subpopulation was present in WT mice upon hTDP-43 expression. However, CD11c+ microglia were not observed in TREM2 KO mice expressing GFP-hTDP-43. Data are represented as mean \pm SEM. Significances were calculated using Two-way ANOVA, Tukey's post-hoc analysis (c-e, h and i) and student $t$ test (I); n.s., not significant; ${ }^{\mathrm{P}}<$ $0.05,{ }^{*} \mathrm{P}<0.01, \star \star \star \mathrm{P}<0.001$. 

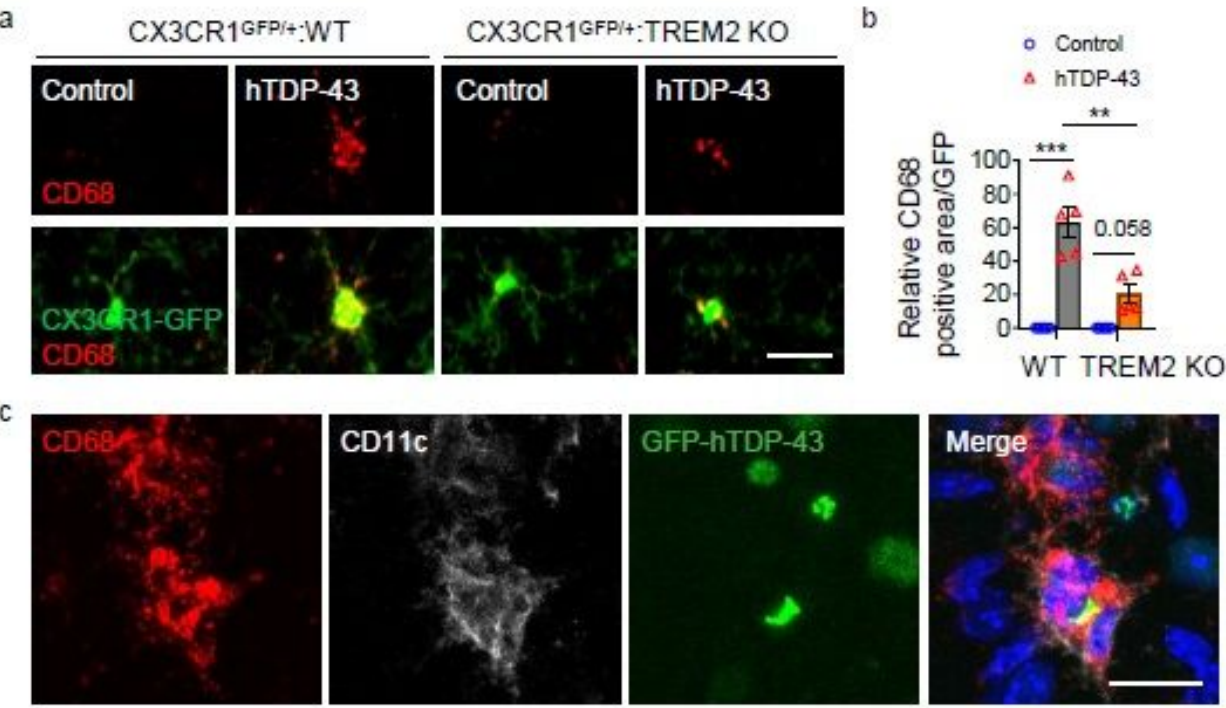

d

WT
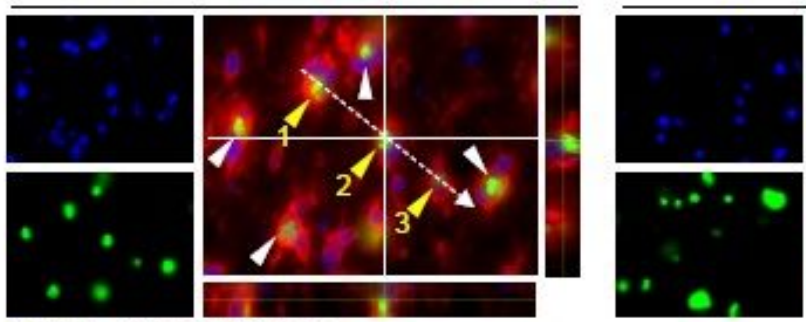

TREM2 KO
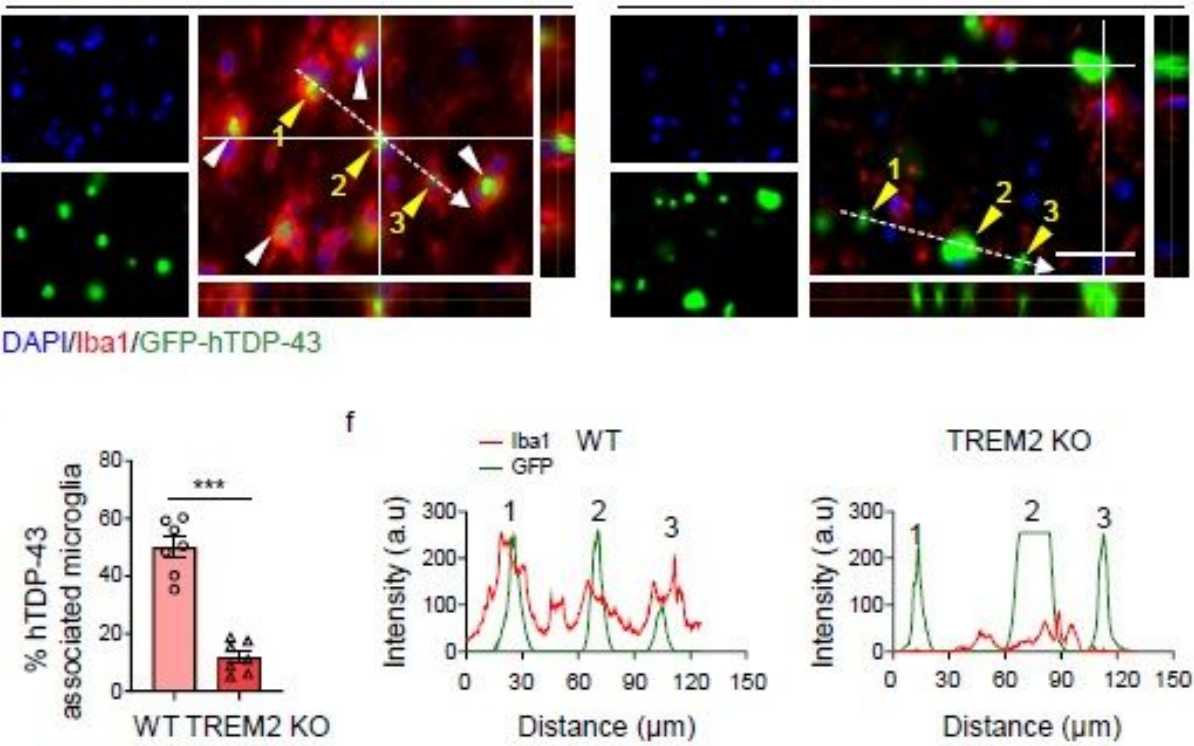

DAPI/ba1/GFP-hTDP-43

g
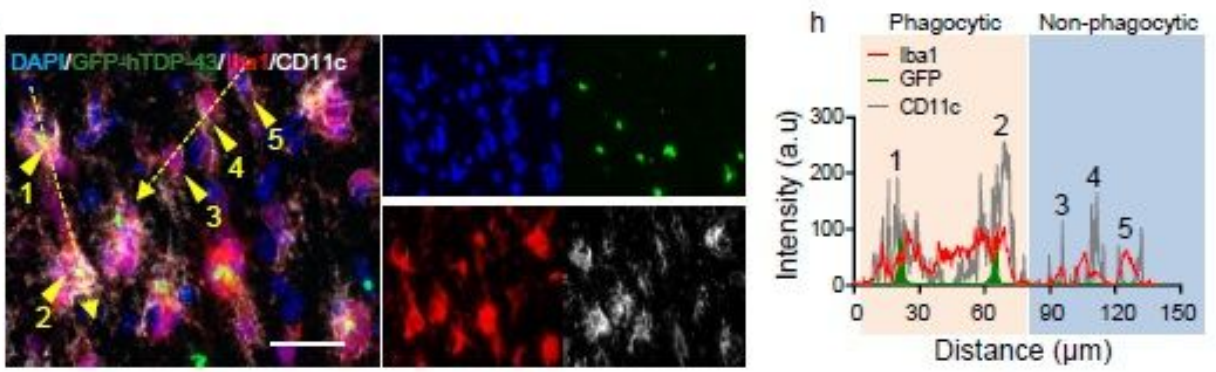

Figure 4

TREM2 deficiency impairs microglial phagocytosis of pathological hTDP-43 protein. hTDP-43 or GFPhTDP-43 was expressed in the primary motor cortex of 2-month-old mice via stereotactic intracerebral injection of AAV9.CAG.hTDP-43 or AAV9.CAG.hTDP43-GFP (AAV9.CAG.Empty or AAV9.CAG.GFP as control). a, Representative images of CD68 expression (red) in microglia (CX3CR1-GFP, green) in the primary motor cortex of indicated groups at $14 \mathrm{dpi}$; Scale bar, $100 \mu \mathrm{m}$. b, Quantification of relative CD68 
positive area in the primary motor cortex of indicated groups at $14 \mathrm{dpi}(\mathrm{n}=5$ per group). Results show hTDP-43 induced increase of CD68 expression in WT but not in TREM2 KO mice. c, Representative images of CD68 (red) and CD11c (white) expression in microglia phagocytosing GFP-hTDP-43 (green) in the primary motor cortex of WT mice at $14 \mathrm{dpi}$; Scale bar, $10 \mu \mathrm{m}$. d, Representative images of microglia (Iba1, red) phagocytosis of GFP-hTDP-43 (green) in the primary motor cortex of WT but not TREM2 KO mice at $28 \mathrm{dpi}$, as indicated by the arrowheads; Scale bar, $20 \mu \mathrm{m}$. e, Quantification of hTDP-43 associated microglia (Iba1) in the primary motor cortex of WT and TREM2 KO mice at $28 \mathrm{dpi}$ ( $\mathrm{n}=7$ per group). Result indicates decreased hTDP-43 associated microglia in TREM2 KO mice. $\mathrm{f}$, Analysis of co-localization of Iba1 (red curves) and GFP-hTDP-43 (green curves) in the primary motor cortex of WT and TREM2 KO mice at 28 dpi. Fluorescence intensity profiles of Iba1 and GFP-hTDP-43 show the distribution of fluorescence across the white dotted arrows in (d). $g$, Representative images of co-localization of CD11c (white) with Iba1 (red) in microglia phagocytosing GFP-hTDP-43 (green) in the primary motor cortex of WT mice at $28 \mathrm{dpi}$; Scale bar, $10 \mu \mathrm{m}$. h, Analysis of co-localization of Iba1 (red curves), CD11c (grey curves) and GFP-hTDP-43 (green curves) in the primary motor cortex of WT mice at 28 dpi. Fluorescence intensity profiles of Iba1, CD11c, and GFP-hTDP-43 show the distribution of fluorescence across the yellow dotted arrows in (g). Data are represented as mean \pm SEM. Significances were calculated using Two-way ANOVA, Tukey's post-hoc analysis (b) and student $t$ test (e); n.s., not significant; ${ }^{*} \mathrm{P}<0.05,{ }^{*} \mathrm{P}<$ $0.01, * \star \star P<0.001$. 
a

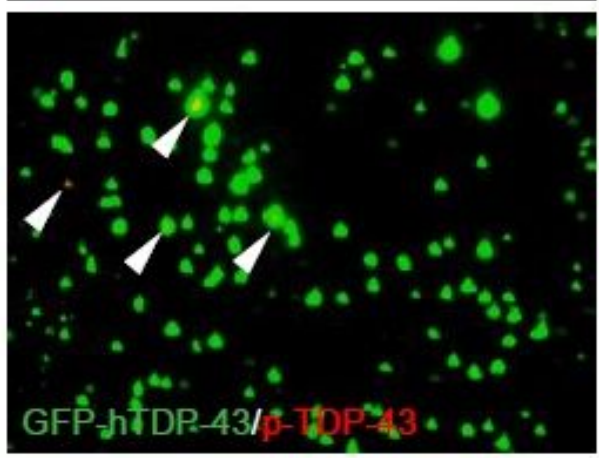

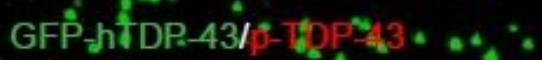

TREM2 KO
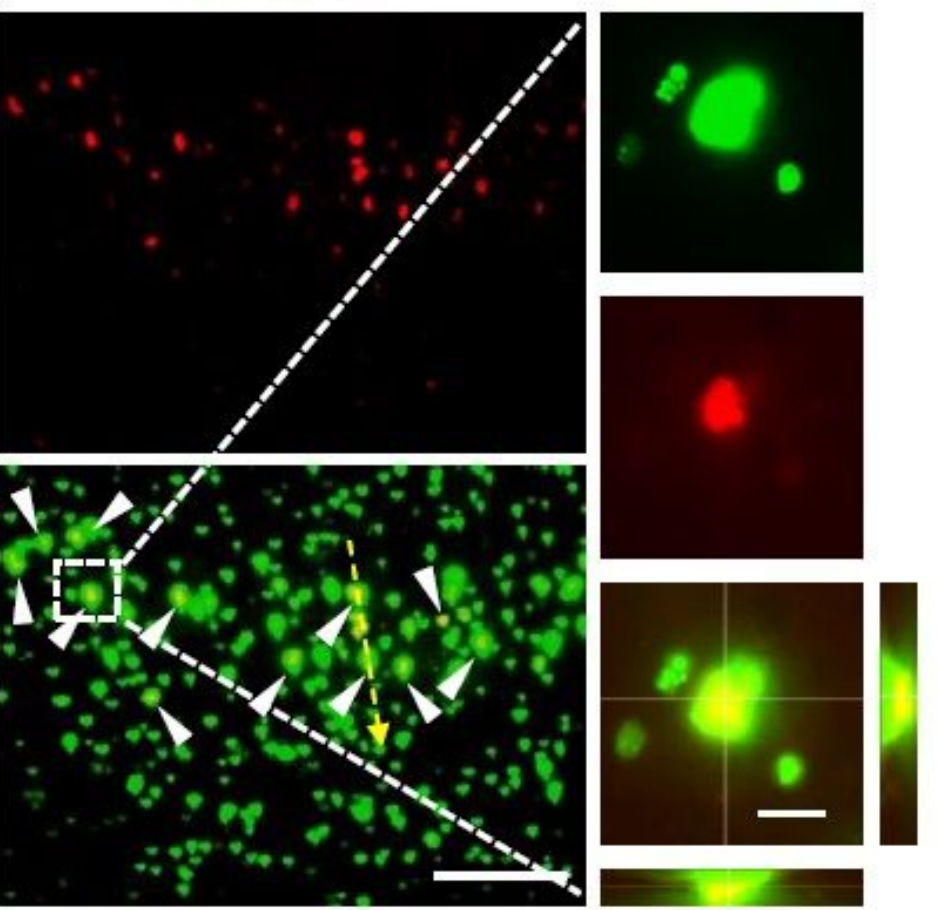

b

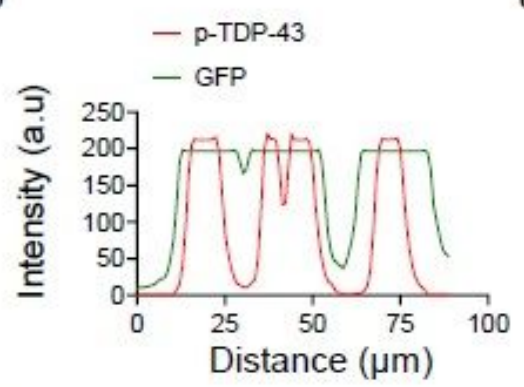

c

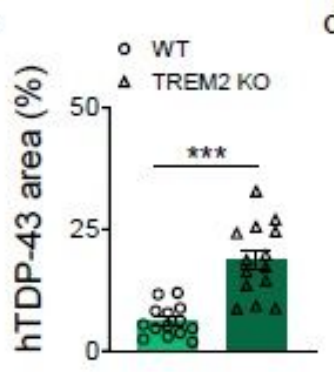

d

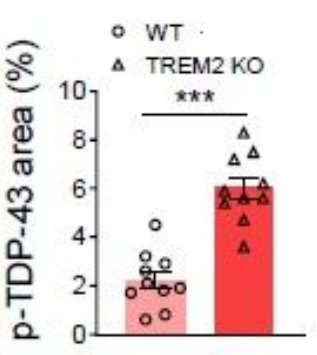

$\mathrm{e}$

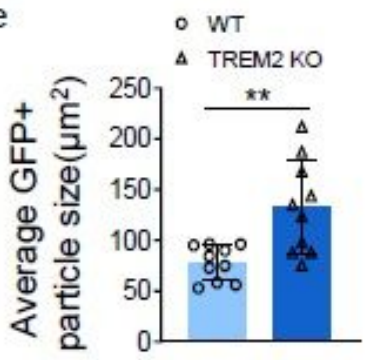

f

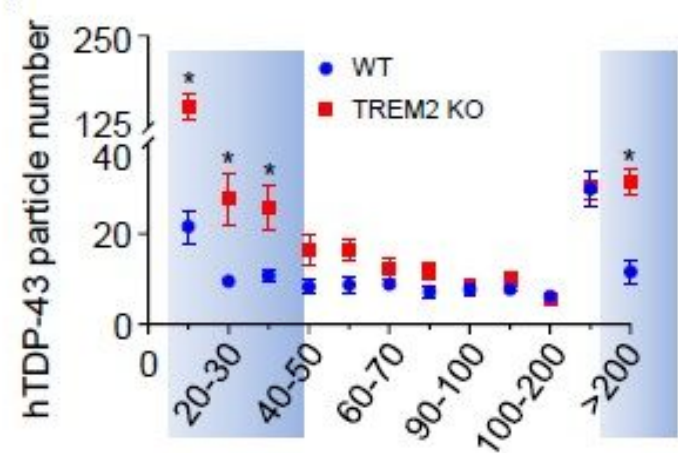

Particle size $\left(\mu \mathrm{m}^{2}\right)$ g

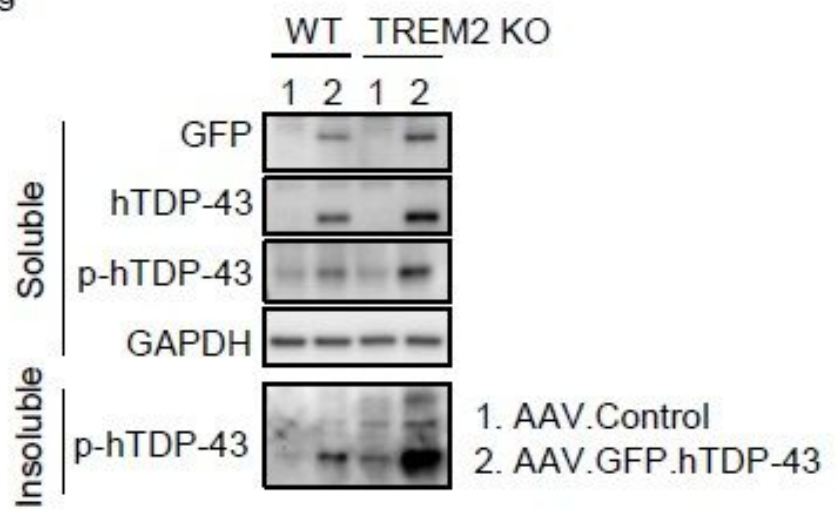

\section{Figure 5}

TREM2 deficiency leads to increased accumulation of pathological hTDP-43 protein. a, Representative images of co-localization of p-TDP-43 (red) with GFP-hTDP-43 in the primary motor cortex of WT and TREM2 KO mice at $28 \mathrm{dpi}$, as indicated by white arrowheads; Scale bar, $100 \mu \mathrm{m}$. High magnification images as indicated by area in dotted white box showing on the right; Scale bar, $10 \mu \mathrm{m}$. b. Analysis of colocalization of p-TDP-43 (red curves) and GFP-hTDP-43 (green curves) in the primary motor cortex of WT 
and TREM2 $\mathrm{KO}$ mice at $28 \mathrm{dpi}$. Fluorescence intensity profiles of p-TDP-43 and GFP-hTDP-43 show the distribution of fluorescence across the yellow dotted arrow in (a). c-e, Quantification of hTDP-43 area (c, $\mathrm{n}=14$ per group), $\mathrm{p}$-TDP-43 area ( $\mathrm{d}, \mathrm{n}=10$ per group) and GFP+ particle size (e, $\mathrm{n}=10$ per group) in the primary motor cortex of WT and TREM2 KO mice at $28 \mathrm{dpi}$. Results indicate higher hTDP-43, p-TDP-43 and GFP+ in TREM2 KO mice. f, hTDP-43 particle size distribution in the primary motor cortex of WT and TREM2 KO mice at 28 dpi. Particles with size below $40 \mu \mathrm{m} 2$ or over $200 \mu \mathrm{m} 2$ are highlighted by blue ( $\mathrm{n}=9$ per group). g, GFP-hTDP-43 and p-hTDP-43 (Ser403/404) immunoblots of the soluble fractions and Sarkosyl-insoluble fraction from primary motor cortex of WT and TREM2 KO mice at $28 \mathrm{dpi}$. Western blots were independently repeated four times ( $n=4$ per group). GAPDH was used as loading control. TREM2 KO mice show high level of GFP-hTDP-43 and p-hTDP-43 as compared to WT mice. Data represented as mean \pm SEM. Significance was calculated using student $t$ test; $n$.s., not significant; $* \mathrm{P}<$ $0.05, * * P<0.01$. 

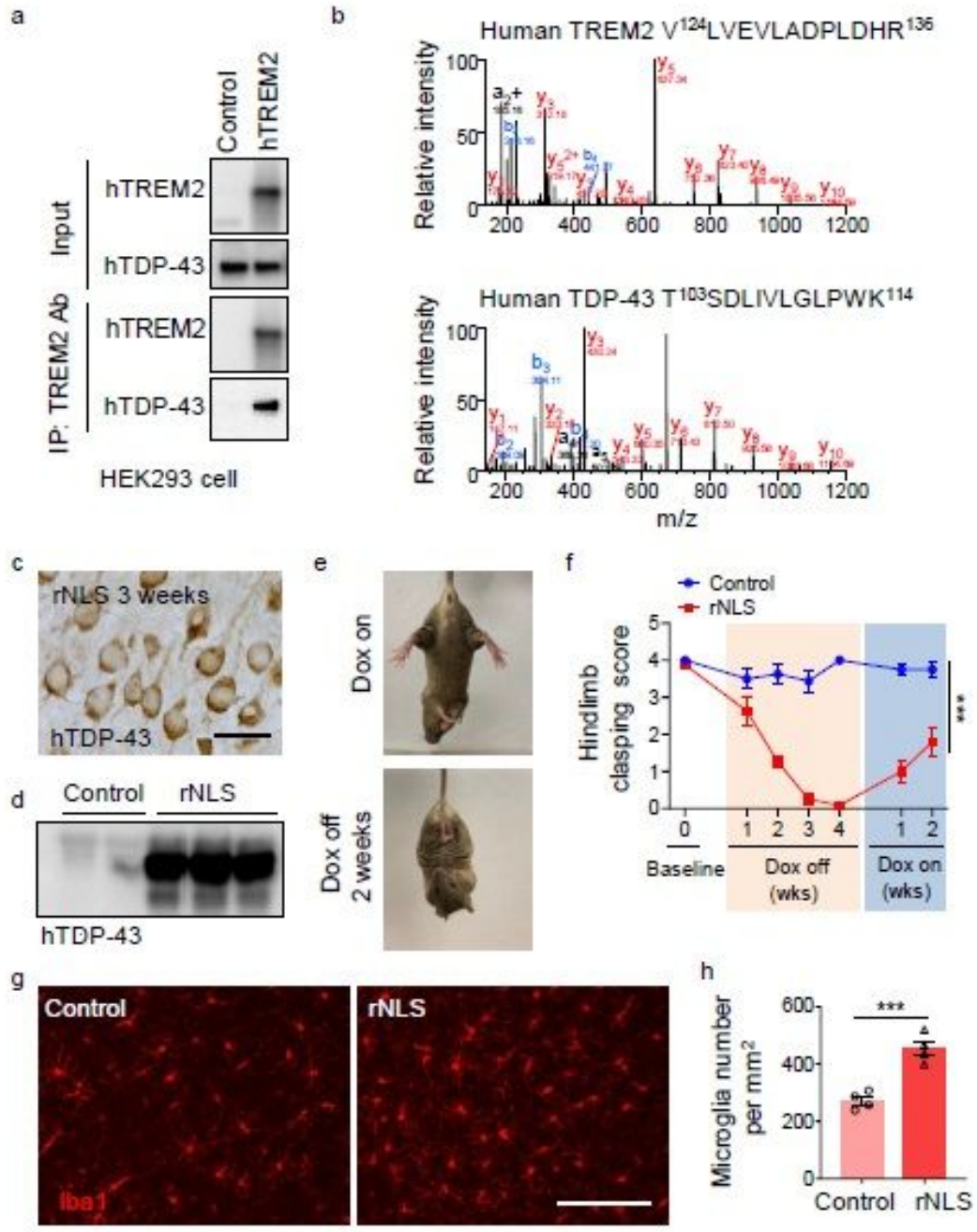

4 weeks DOX off +2 weeks DOX on

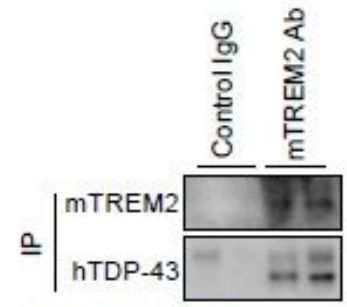

INLS mouse brain:

4 weeks DOX off +2 weeks DOX on

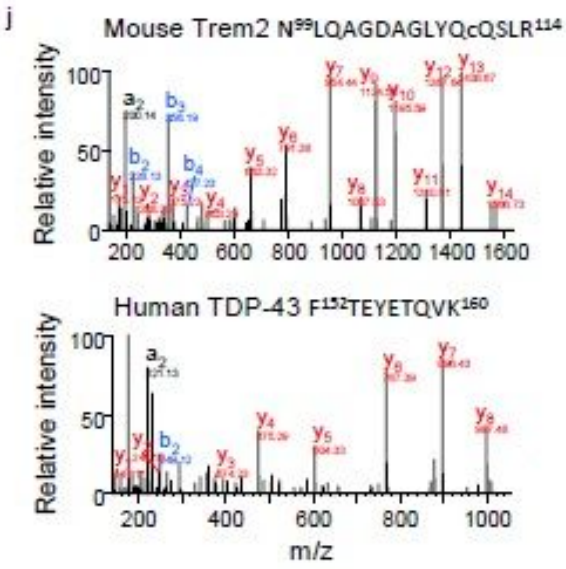

\section{Figure 6}

hTDP-43 interacts with TREM2 in vitro and in vivo in mouse brain. a, hTDP-43 was coimmunoprecipitated with myc-hTREM2 in HEK 293 cells overexpressing hTREM2. Experiments were independently repeated three times. b, Annotated MS/MS spectra of V124LVEVLADPLDHR136 from hTREM2 and T103SDLIVLGLPWK114 from hTDP-43 identified in anti-hTREM2 immunoprecipitates from HEK 293 cells. c, Representative images of hTDP-43 expression in cytosol in the primary motor cortex of 
rNLS mice at 3 weeks off DOX diet; Scale bar, $20 \mu \mathrm{m}$. d, hTDP-43 immunoblots of primary motor cortex of rNLS mice at 3 weeks off DOX diet. e, rNLS mice showed clasping phenotype at 2 weeks off DOX diet. $f$, Hindlimb clasping response scores over 4 weeks off DOX diet followed by 2 weeks DOX on ( $n=12$ per group). g, Representative images of microglia (lba1, red) in the primary motor cortex of indicated groups at 4 weeks off DOX diet followed with 2 weeks DOX on. h, Quantification of microglial density $(n=10$ per group) in the primary motor cortex of indicated groups at 4 weeks off DOX diet followed by 2 weeks DOX on. Results indicate increased microglia number in rNLS mice. i, Endogenous mTREM2 was coimmunoprecipitated with hTDP-43 in the motor cortex of rNLS mice using mTREM2 antibody at 4 weeks off DOX diet followed with 2 weeks DOX on. Experiments were independently repeated three times. j, Annotated MS/MS spectra of N99LQAGDAGLYQcQSLR114 (c is carbaminomethylated cysteine) from mouse TREM2 and F152TEYETQVK160 from hTDP-43 in anti-mTREM2 immunoprecipitates from rNLS mouse cortex tissue at 4 weeks off DOX diet followed by 2 weeks DOX on. Data represented as mean \pm SEM. Significances were calculated using Two-way ANOVA, Tukey's post-hoc analysis ( $\mathrm{f}$ ) and student $\mathrm{t}$ test (h); n.s., not significant; ${ }^{*} P<0.05,{ }^{*} \mathrm{P}<0.01,{ }^{\star \star *} \mathrm{P}<0.001$. 
a

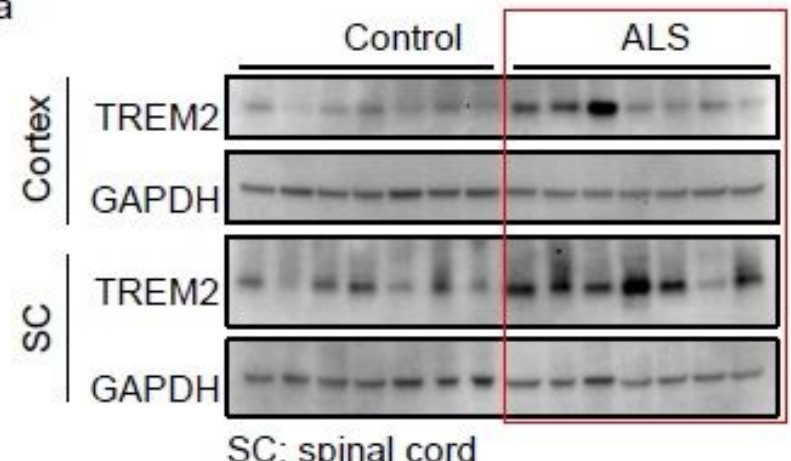

SC: spinal cord
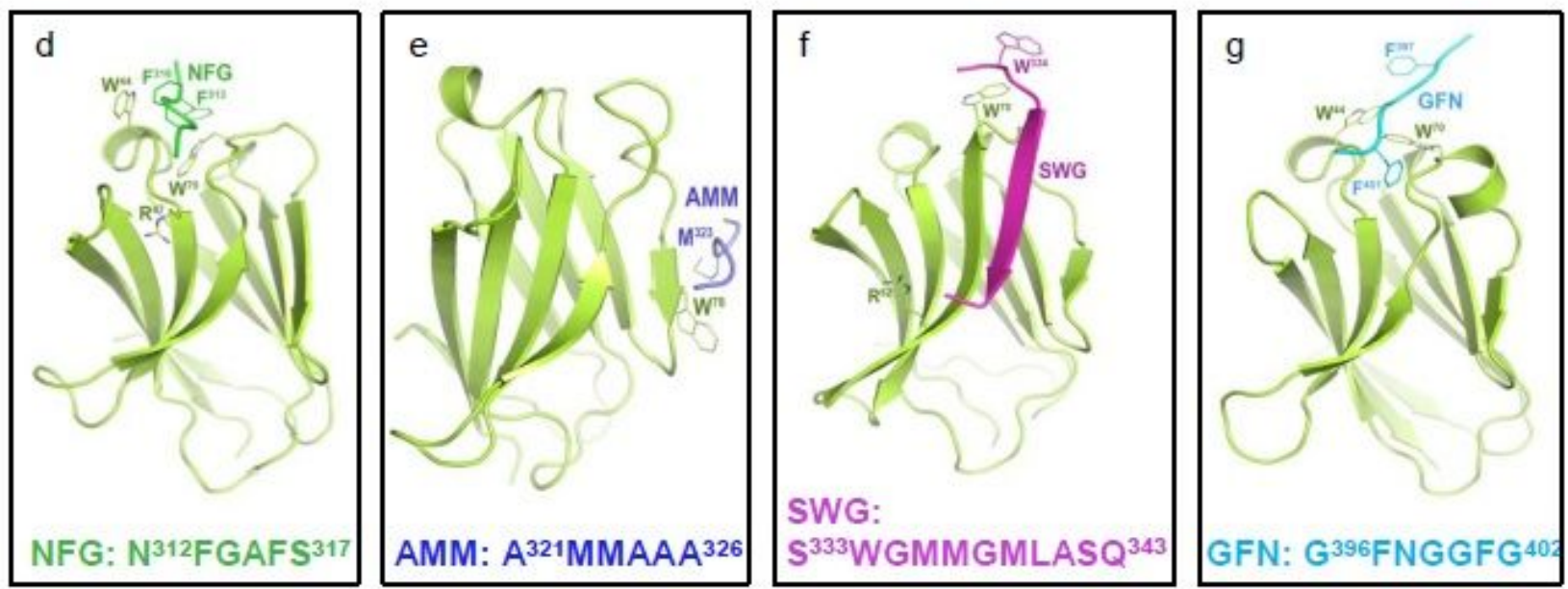

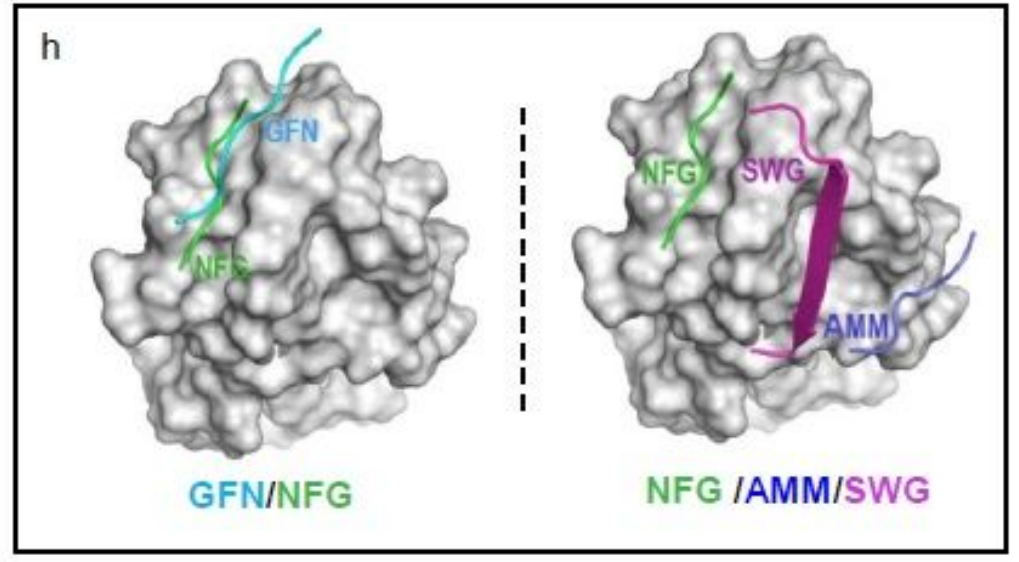

\section{Figure 7}

hTDP-43 interacts with TREM2 in human tissues and in silico. a, TREM2 immunoblots of frozen autopsied spinal cord specimens of ALS patients and age matched controls. b, Quantification of TREM2 level relative to GAPDH ( $n=12$ for control, $n=16$ for ALS patients). Results indicate increased expression of TREM2 in ALS patient samples as compared to non-neurological controls. c, Endogenous TDP-43 of frozen autopsied spinal cord specimens from ALS patients was co-immunoprecipitated with TREM2. 
Experiments were independently repeated three times. $d$-g, Cartoon models of the human TREM2 extracellular ligand-binding domain in complex with four low-complexity domain fragments of TDP-43. h, Surface model of the TREM2 complex showing overlapping binding sites of GFN and NFG and distinct binding sites of NFG, AMM, and SWG. (NFG: N312FGAFS317; AMM: A321MMAAA326; SWG:

S333WGMMGMLASQ343; GFN: G396FNGGFG402). Data represented as mean \pm SEM. Significances were calculated using student $t$ test; $n$.s., not significant; ${ }^{\star} P<0.05$, $* \star P<0.01,{ }^{\star \star \star} P<0.001$. 